\title{
Highly Strained 1,8-Naphthalene Bridged Cyclic Oligophenylenes and Their Open-shell Diradical Dications
}

Chunchen Liu, Guangwu Li, Hoa Phan, Ya Zou, Xuefeng Lu, and Jishan Wu*

\section{Table of Contents}

1. Experimental section....................................................... 2

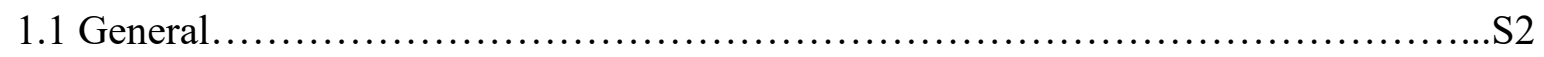

1.2 Synthetic procedures and characterization data.................................. 3

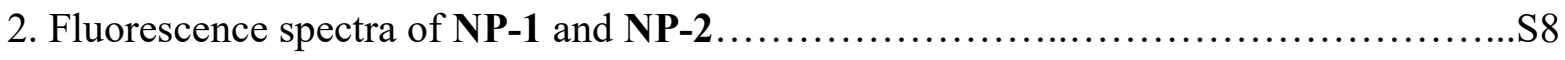

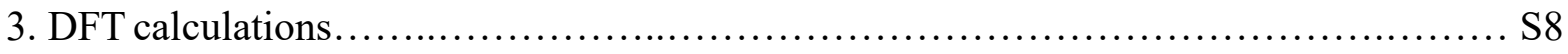

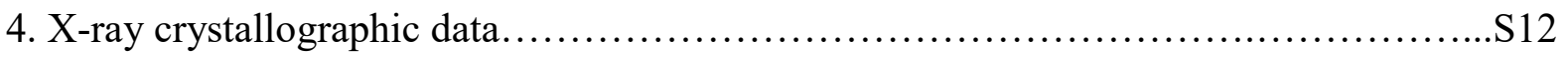

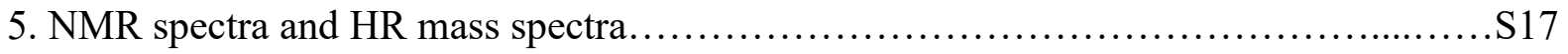

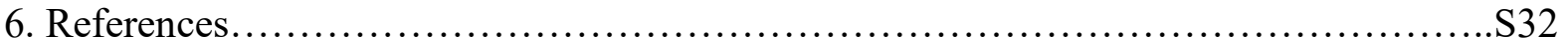

7. Cartesian coordinates and energies of optimised geometries.........................S33 


\section{Experiment Section}

\subsection{General}

All reagents and starting materials were obtained from commercial suppliers and used without further purification unless otherwise specified. Anhydrous toluene and THF were distilled from sodium-benzophenone immediately prior to use. Anhydrous dichloromethane (DCM) was distilled from $\mathrm{CaH}_{2}$. All reaction conditions dealing with air- and moisture sensitive compounds were carried out in a dry reaction vessel under a nitrogen atmosphere. 1,8-Dibromo-2,7-bis(octyloxy)naphthalene $(\mathbf{1})^{1}$, (4-(trimethylsilyl)phenyl)boronic acid (2a) and (4'-(trimethylsilyl)-[1,1'-biphenyl]4-yl)boronic acid (2b) ${ }^{2}$ were prepared according to the similar procedures reported in literatures. The ${ }^{1} \mathrm{H}$ NMR and ${ }^{13} \mathrm{C}$ NMR spectra were recorded in solution of $\mathrm{CDCl}_{3}$ and $\mathrm{CD}_{2} \mathrm{Cl}_{2}$ on Bruker DPX 300 or DRX 500 NMR spectrometers. All chemical shifts are quoted in ppm, relative to tetramethylsilane, using the residual solvent peak as a reference standard. The following abbreviations were used to explain the multiplicities: $\mathrm{s}$ $=$ singlet, $\mathrm{d}=$ doublet, $\mathrm{t}=$ triplet and $\mathrm{m}=$ multiple. High-resolution Atmospheric Pressure Chemical Ionization Mass Spectrometry (HR-APCI MS) measurements were recorded on a Bruker amazon equipment. UV-vis-NIR absorption spectra were recorded on a Shimadzu UV3600 spectrophotometer. Cyclic voltammetry measurements were performed in anhydrous DCM on a CHI 620C electrochemical analyzer with $0.1 \mathrm{M} n-\mathrm{Bu}_{4} \mathrm{NPF}_{6}$ as supporting electrolyte at a scan rate of $50 \mathrm{mV} / \mathrm{s}$ at room temperature under inert atmosphere. A gold disk, platinum and $\mathrm{Ag} / \mathrm{AgCl}$ were used as working electrode, counting electrode and reference electrode, respectively. The potential was externally calibrated against the ferrocene/ferrocenium couple. Continuous wave X-band ESR spectra were obtained with a JEOL (FA200) spectrometer using a variable temperature liquid nitrogen cryostat.

ESR measurements were conducted for the solution in DCM from the freezing temperature to $118 \mathrm{~K}$, and the data are fitted by modified Bleaney-Bowers equation:

$$
I T=\frac{C}{k_{B}\left[3+\exp \left(-2 J / k_{B} T\right)\right]}
$$

Where $-2 J$ is correlated to the singlet-to-triplet excitation energy. 


\subsection{Synthetic Procedures and Characterization Data}

\section{1,8-Dibromo-2,7-bis(octyloxyl)naphthalene (1)}

A solution of $N$-bromosuccinimide (NBS) $(1.85 \mathrm{~g}, 10.4 \mathrm{mmol})$ in $\mathrm{CHCl}_{3}(25 \mathrm{~mL})$ was mixed with pyridine $(0.82 \mathrm{~g}, 10.4 \mathrm{mmol})$ under nitrogen. The mixture was heated at reflux for 1 hour (all NBS had dissolved and the solution turned from colorless to orange). A solution of 2,7dioctyloxynaphthalene $(1.0 \mathrm{~g}, 2.6 \mathrm{mmol})$ in chloroform $(\sim 5 \mathrm{~mL})$ was added dropwise, followed by heating at reflux for 9 hours. After cooling to room temperature and evaporation, the crude reaction mixture was purified by silica gel chromatography (hexane : $\mathrm{DCM}=10: 1$ ) to provide a white amorphous solid product (1) $(0.96 \mathrm{~g}, 68 \%) .{ }^{1} \mathrm{H}$ NMR (300 $\left.\mathrm{MHz}, \mathrm{CDCl}_{3}\right): \delta \mathrm{ppm} 7.67$ $(\mathrm{d}, J=8.9 \mathrm{~Hz}, 1 \mathrm{H}), 7.10(\mathrm{~d}, J=8.9 \mathrm{~Hz}, 1 \mathrm{H}), 4.13(\mathrm{t}, J=6.5 \mathrm{~Hz}, 2 \mathrm{H}), 2.01-1.75(\mathrm{~m}, 2 \mathrm{H}), 1.55$ $(\mathrm{d}, J=2.6 \mathrm{~Hz}, 2 \mathrm{H}), 1.30$ (s, 8H), 0.90 (s, 3H). ${ }^{13} \mathrm{C} \mathrm{NMR}\left(75 \mathrm{MHz}, \mathrm{CDCl}_{3}\right): \delta \mathrm{ppm} \mathrm{156.3,} \mathrm{132.1,}$ 129.9, 127.6, 113.2, 107.0, 70.6, 32.0, 29.6, 29.5, 29.4, 26.2, 22.8, 14.2. HRMS (ESI) m/z: [M $+\mathrm{Na}]^{+}$Calcd for $\mathrm{C} 26 \mathrm{H} 38 \mathrm{Br} 2 \mathrm{O} 2 \mathrm{Na} 563.1239$; Found 563.1235.

\section{((2,7-Bis(octyloxy)naphthalene-1,8-diyl)bis(4,1-phenylene))bis(trimethylsilane) (3a)}

Compound 1 (1.08 g, 2 mmol), (4-(trimethylsilyl)phenyl)boronic acid (2a) (1.16 g, 6 mmol), $\mathrm{K}_{2} \mathrm{CO}_{3}(2.21 \mathrm{~g}, 16 \mathrm{mmol})$ and $\mathrm{Pd}\left(\mathrm{PPh}_{3}\right)_{4}(120 \mathrm{mg}, 0.1 \mathrm{mmol})$ were added to the round-bottom flask and protected under nitrogen atmosphere. Then anhydrous toluene $(30 \mathrm{~mL})$ and water $(3$ $\mathrm{mL}$ ) were added to the flask, and the reaction mixture was heated to reflux for 2 days. After cooling to room temperature, the reaction mixture was extracted with DCM for 3 times, and the combined organic layer was dried with anhydrous sodium sulfate. After the filtration and evaporation, the obtained crude mixture was purified via silica gel chromatography (hexane : $\mathrm{DCM}=10: 1)$ to give the final product as white solid (3a) (650 mg, 48\%). ${ }^{1} \mathrm{H}$ NMR (300 MHz, $\left.\mathrm{CD}_{2} \mathrm{Cl}_{2}\right): \delta$ ppm $7.82(\mathrm{~d}, J=8.9 \mathrm{~Hz}, 1 \mathrm{H}), 7.20(\mathrm{~d}, J=9.0 \mathrm{~Hz}, 1 \mathrm{H}), 7.09-6.92(\mathrm{~m}, 2 \mathrm{H}), 3.87(\mathrm{t}$, $J=6.3 \mathrm{~Hz}, 2 \mathrm{H}), 1.50-1.38(\mathrm{~m}, 2 \mathrm{H}), 1.31-1.07(\mathrm{~m}, 10 \mathrm{H}), 0.87$ (t, $J=6.9 \mathrm{~Hz}, 3 \mathrm{H}), 0.22(\mathrm{~s}, 9 \mathrm{H})$. ${ }^{13} \mathrm{C}$ NMR $\left(75 \mathrm{MHz}, \mathrm{CD}_{2} \mathrm{Cl}_{2}\right): \delta$ ppm 156.0, 139.6, 136.6, 134.5, 131.8, 131.4, 129.6, 126.8, 126. 6, 113.6, 70.1, 32.3, 30.2, 29.8, 29.6, 26.2, 23.1, -0.8. HRMS (ESI) m/z: $[\mathrm{M}+\mathrm{Na}]^{+} \mathrm{Calcd}$ for C44H64O2Si2Na 703.4445; Found 703.4451. 
((2,7-Bis(octyloxy)naphthalene-1,8-diyl)bis([1,1'-biphenyl]4',4-diyl))bis(trimethylsilane) (3b)

Compound 3b (188 mg, white amorphous solid, 46\%) was prepared with the similar procedures as 3a. ${ }^{1} \mathrm{H}$ NMR (300 MHz, $\left.\mathrm{CD}_{2} \mathrm{Cl}_{2}\right)$ : $\delta$ ppm $7.85(\mathrm{~d}, J=8.9 \mathrm{~Hz}, 1 \mathrm{H}), 7.43(\mathrm{~d}, J=8.0 \mathrm{~Hz}, 2 \mathrm{H})$, 7.38-7.29 (m, 2H), $7.23(\mathrm{~d}, J=9.0 \mathrm{~Hz}, 1 \mathrm{H}), 7.13-7.04(\mathrm{~m}, 2 \mathrm{H}), 6.88-6.77(\mathrm{~m}, 2 \mathrm{H}), 3.88(\mathrm{t}, J=$ $6.3 \mathrm{~Hz}, 2 \mathrm{H}), 1.44(\mathrm{t}, J=6.8 \mathrm{~Hz}, 2 \mathrm{H}), 1.08(\mathrm{~d}, J=10.4 \mathrm{~Hz}, 10 \mathrm{H}), 0.79(\mathrm{t}, J=6.9 \mathrm{~Hz}, 3 \mathrm{H}), 0.31$ (s, 9H). ${ }^{13} \mathrm{C}$ NMR (75 MHz, $\left.\mathrm{CD}_{2} \mathrm{Cl}_{2}\right): \delta$ ppm 155.9, 142.2, 138.4, 138.0, 137.9, 133.9, 133.6, $132.1,129.8,126.7,126.5,126.2,125.5,113.7,70.2,31.9,29.6,29.4,25.9,22.7,14.2,0.8$. HR MS (ESI) m/z: [M + Na] ${ }^{+}$Calcd for C56H72O2Si2Na 855.5071; Found 855.5076.

\section{1,8-Bis(4-iodophenyl)2,7-bis(octyloxy)naphthalene (4a)}

Compound 3a (150 mg, 0.22mmol) was dissolved in anhydrous dichloromethane (15 mL) and then the solution was cooled to $-78^{\circ} \mathrm{C}$. Iodine monochloride solution $(0.44 \mathrm{~mL})$ in DCM $(1 \mathrm{M})$ was added dropwise to the reaction solution. After 1 hour, the reaction was warmed up to room temperature and aqueous solution of sodium thiosulfate pentahydrate was added to quench the reaction. Then organic phase was separated and dried over anhydrous sodium. After filtration and evaporation, the crude mixture was purified with silica gel chromatography (hexane : DCM $=5: 1)$ to afford the final product as white amorphous solid (116 mg, 67\%). ${ }^{1} \mathrm{H}$ NMR (300 $\left.\mathrm{MHz}, \mathrm{CDCl}_{3}\right): \delta \mathrm{ppm} 7.83(\mathrm{~d}, J=8.9 \mathrm{~Hz}, 1 \mathrm{H}), 7.29(\mathrm{~d}, J=8.2 \mathrm{~Hz}, 2 \mathrm{H}), 7.18(\mathrm{~d}, J=8.9 \mathrm{~Hz}$, $1 \mathrm{H}), 6.49(\mathrm{~d}, J=8.2 \mathrm{~Hz}, 2 \mathrm{H}), 3.86(\mathrm{t}, J=6.3 \mathrm{~Hz}, 2 \mathrm{H}), 1.51-1.38(\mathrm{~m}, 2 \mathrm{H}), 1.35-0.98(\mathrm{~m}, 10 \mathrm{H})$, $0.88(\mathrm{t}, J=7.0 \mathrm{~Hz}, 3 \mathrm{H}) .{ }^{13} \mathrm{C} \mathrm{NMR}\left(75 \mathrm{MHz}, \mathrm{CDCl}_{3}\right): \delta \mathrm{ppm} 155.5,137.8,135.8,133.5,132.8$, $129.9,125.7,124.6,113.0,91.1,69.7,31.7,29.2,29.1,29.0,25.7,22.5,14.0$. HRMS (ESI) m/z: $[\mathrm{M}+\mathrm{Na}]^{+}$Calcd for C38H46I2O2Na 811.1587; Found 811.1582.

\section{1,8-Bis(4'-iodo-[1,1'-biphenyl]-4-yl)-2,7-bis(octyloxy)naphthalene (4b)}

Compound $4 \mathbf{b}$ (387 mg, white amorphous solid, 83\%) was prepared with the similar procedures as 4a. ${ }^{1} \mathrm{H}$ NMR (300 MHz, $\left.\mathrm{CDCl}_{3}\right): \delta 7.86(\mathrm{~d}, J=9.0 \mathrm{~Hz}, 1 \mathrm{H}), 7.58(\mathrm{~d}, J=8.4 \mathrm{~Hz}, 2 \mathrm{H}), 7.22$ $(\mathrm{d}, J=9.0 \mathrm{~Hz}, 1 \mathrm{H}), 7.03(\mathrm{~d}, J=8.4 \mathrm{~Hz}, 4 \mathrm{H}), 6.84(\mathrm{~d}, J=8.3 \mathrm{~Hz}, 2 \mathrm{H}), 3.88(\mathrm{t}, J=6.3 \mathrm{~Hz}, 2 \mathrm{H})$, $1.45(\mathrm{t}, J=6.8 \mathrm{~Hz}, 2 \mathrm{H}), 1.22-0.95(\mathrm{~m}, 10 \mathrm{H}), 0.79(\mathrm{t}, J=7.0 \mathrm{~Hz}, 3 \mathrm{H}) .{ }^{13} \mathrm{C}$ NMR $(75 \mathrm{MHz}$, $\left.\mathrm{CDCl}_{3}\right): \delta$ ppm $155.6,140.8,138.1,137.5,136.6,132.3,129.9,128.7,126.0,125.7,125.0$ 
113.4, 110.0, 92.2, 69.9, 31.7, 29.4, 29.2, 29.1, 25.7, 22.6, 14.0. HRMS (ESI) m/z: $[\mathrm{M}+\mathrm{Na}]^{+}$ Calcd for C50H54I2O2Na 963.2213; Found 963.2217.

((2,7-Bis(octyloxy)naphthalene-1,8-diyl)bis(4,1-phenylene))bis(trimethylstannane) (5a)

Compound 4a (100 mg, $0.17 \mathrm{mmol})$ was dissolved in anhydrous tetrahydrofuran $(10 \mathrm{~mL})$ and the solution was cooled to $-78^{\circ} \mathrm{C}$. Then $0.2 \mathrm{~mL}$ of $n$-butyl lithium in hexane $(2.0 \mathrm{M})$ was added dropwise to the solution and the reaction was kept at this temperature for 1.5 hours. After that, $0.44 \mathrm{~mL}$ of trimethyl tin chloride in hexane solution $(1 \mathrm{M})$ was added dropwise into the reaction mixture. The reaction was warmed up to room temperature gradually and stirred overnight. The reaction was quenched by water and extracted with diethyl ester three times. The combined organic layer was dried over anhydrous sodium sulfate. After filtration and evaporation, the crude mixture was recrystallized in methanol to afford the final product as white amorphous solid (99.6 mg, 91\%). ${ }^{1} \mathrm{H}$ NMR (300 MHz, $\left.\mathrm{CDCl}_{3}\right): \delta \mathrm{ppm} 7.79(\mathrm{~d}, J=8.9 \mathrm{~Hz}, 1 \mathrm{H}), 7.17(\mathrm{~d}, J$ $=9.0 \mathrm{~Hz}, 1 \mathrm{H}), 6.97(\mathrm{~d}, J=7.9 \mathrm{~Hz}, 2 \mathrm{H}), 6.81-6.68(\mathrm{~m}, 2 \mathrm{H}) 3.83(\mathrm{t}, J=6.3 \mathrm{~Hz}, 2 \mathrm{H}), 1.42(\mathrm{t}, J=$ $7.0 \mathrm{~Hz}, 2 \mathrm{H}), 1.27-1.06(\mathrm{~m}, 10 \mathrm{H}), 0.85$ (t, $J=7.0 \mathrm{~Hz}, 3 \mathrm{H}), 0.23$ (s, 9H). ${ }^{13} \mathrm{C} \mathrm{NMR}(75 \mathrm{MHz}$, $\left.\mathrm{CDCl}_{3}\right): \delta$ ppm 155.5, 138.4, 137.8, 133.8, 131.2, 129.2, 126.8, 126.2, 113.6, 70.0, 31.8, 29.7, 29.4, 29.2, 25.7, 22.6, 14.1, -9.5. HRMS (ESI) m/z: $[\mathrm{M}+\mathrm{Na}]^{+}$Calcd for C44H64O2Sn2Na 887.2950; Found 887.2953.

\section{((2,7-Bis(octyloxy)naphthalene-1,8-diyl)bis([1,1'-biphenyl]-4',4-}

diyl))bis(trimethylstannane) (5b)

Compound 5b (286 mg, white amorphous solid, 89\%) was prepared with the similar procedures with compound 5a. ${ }^{1} \mathrm{H}$ NMR $\left(300 \mathrm{MHz}, \mathrm{CD}_{2} \mathrm{Cl}_{2}\right): \delta 7.87(\mathrm{~d}, J=9.0 \mathrm{~Hz}, 1 \mathrm{H}), 7.48-7.30$ (m, $3 \mathrm{H}), 7.28-7.21(\mathrm{~m}, 2 \mathrm{H}), 7.09(\mathrm{~d}, J=8.1 \mathrm{~Hz}, 3.0,2 \mathrm{H}), 6.92-6.74(\mathrm{~m}, 2 \mathrm{H}), 4.01-3.83(\mathrm{~m}, 2 \mathrm{H})$, $1.48(\mathrm{~d}, J=6.3 \mathrm{~Hz}, 2 \mathrm{H}), 1.12(\mathrm{~s}, 10 \mathrm{H}), 0.87(\mathrm{~d}, J=7.1 \mathrm{~Hz}, 3 \mathrm{H}), 0.31(\mathrm{~d}, J=4.4 \mathrm{~Hz}, 9 \mathrm{H}) .{ }^{13} \mathrm{C}$ NMR (75 MHz, $\left.\mathrm{CDCl}_{3}\right): \delta$ ppm 155.9, 141.7, 138.1, 137.7, 136.0, 133.6, 132.1, 129.8, 128.5, $127.3,127.1,126.7,125.5,113.7,70.2,31.9,31.1,29.9,29.6,29.3,25.9,22.7,14.2$. HRMS (ESI) $\mathrm{m} / \mathrm{z}:[\mathrm{M}+\mathrm{Na}]^{+}$Calcd for C56H72O2Sn2Na 1039.3576; Found 1039.3571.

((2,7-Bis(octyloxy)naphthalene-1,8-diyl)bis(1,1':4',1'’-terphenyl]-4',4diyl))bis(trimethylsilane) (3c) 
Compound 3c (1.2 g, white amorphous solid, 57\%) was prepared with the similar procedures with compound 3a. ${ }^{1} \mathrm{H}$ NMR (300 MHz, $\left.\mathrm{CDCl}_{3}\right)$ : $\delta \mathrm{ppm} 7.84(\mathrm{~d}, J=6.0 \mathrm{~Hz}, 1 \mathrm{H}), 7.61(\mathrm{~m}, 4 \mathrm{H})$, 7.48-7.39 (m, 4H), 7.23-7.19 (m, 1H), $7.14(\mathrm{~d}, J=6.2 \mathrm{~Hz}, 2 \mathrm{H}), 6.86(\mathrm{~d}, J=6.1 \mathrm{~Hz}, 2 \mathrm{H}), 3.87$ $(\mathrm{t}, J=4.6 \mathrm{~Hz}, 2 \mathrm{H}), 1.44(\mathrm{~m}, 2 \mathrm{H}), 1.07(\mathrm{~m}, 10 \mathrm{H}), 0.77(\mathrm{t}, J=5.2 \mathrm{~Hz}, 3 \mathrm{H}), 0.31(\mathrm{~s}, 9 \mathrm{H}) .{ }^{13} \mathrm{C}$ NMR (75 MHz, $\left.\mathrm{CDCl}_{3}\right): \delta$ ppm 155.7, 141.3, 140.5, 139.3, 139.0, 137.7, 137.3, 134.0, 133.8, 133.6, 132.1, 129.8, 127.4, 127.1, 126.4, 126.3, 125.3, 113.6, 70.0, 31.8, 29.4, 29.2, 29.2, 25.8, 22.6, 14.0. HRMS (ESI) m/z: $[\mathrm{M}+\mathrm{Na}]^{+}$Calcd for C68H80O2Si2Na 1007.5697; Found 1007.5692. Single crystal of was obtained by slow diffusion of methanol into its solution in DCM, so its structure was further confirmed by X-ray crystallographic analysis.

\section{1,8-bis(4'-iodo-[1,1':4'1'’-terphenyl]-4-yl)-2,7-bis(octyloxy)naphthalene (4c)}

Compound 4c (898 mg, white amorphous solid, 86\%) was prepared with the similar procedures with compound 4a. ${ }^{1} \mathrm{H}$ NMR $\left(300 \mathrm{MHz}, \mathrm{CD}_{2} \mathrm{Cl}_{2}\right): \delta \mathrm{ppm} 7.89(\mathrm{~d}, J=6.7 \mathrm{~Hz}, 1 \mathrm{H}), 7.72(\mathrm{~d}, \mathrm{~J}=$ $6.3 \mathrm{~Hz}, 2 \mathrm{H}), 7.40(\mathrm{~m}, 4 \mathrm{H}), 7.73-7.24(\mathrm{~m}, 3 \mathrm{H}), 7.15(\mathrm{~d}, J=6.2 \mathrm{~Hz}, 2 \mathrm{H}), 6.87(\mathrm{~d}, J=6.2 \mathrm{~Hz}$, 2H), $3.93(\mathrm{t}, J=4.8 \mathrm{~Hz}, 2 \mathrm{H}), 1.48(\mathrm{t}, J=4.7 \mathrm{~Hz}, 2 \mathrm{H}), 1.13(\mathrm{~m}, 10 \mathrm{H}), 0.80(\mathrm{t}, J=5.1 \mathrm{~Hz}, 3 \mathrm{H})$. ${ }^{13} \mathrm{C}$ NMR $\left(75 \mathrm{MHz}, \mathrm{CDCl}_{3}\right): \delta$ ppm 155.9, 141.5, 141.0, 140.7, 138.0, 133.9, 132.4, 132.2, $129.9,128.8,127.5,127.3,126.9,126.5,126.4,126.2,125.4,113.7,70.2,31.9,31.8,29.6$, 29.4, 25.9, 22.8, 14.2. HRMS (ESI) m/z: $[\mathrm{M}+\mathrm{Na}]^{+}$Calcd for C62H62I2O2Na 1115.2839; Found 1115.2842.

\section{((2,7-bis(octyloxy)naphthalene-1,8-diyl)bis(1,1':4',1''-terphenyl]-4',,4-}

\section{diyl))bis(trimethylstannane) (5c)}

Compound 5c (528 mg, light yellow amorphous solid, 63\%) was prepared with the similar procedures with compound 5a. ${ }^{1} \mathrm{H}$ NMR $\left(300 \mathrm{MHz}, \mathrm{CD}_{2} \mathrm{Cl}_{2}\right): \delta \mathrm{ppm} 7.89(\mathrm{~d}, J=6.7 \mathrm{~Hz}, 1 \mathrm{H})$, 7.56-7.32 (m, 8H), $7.26(\mathrm{~d}, J=6.7 \mathrm{~Hz}, 1 \mathrm{H}), 7.17(\mathrm{~d}, J=6.2 \mathrm{~Hz}, 2 \mathrm{H}), 6.87(\mathrm{~d}, J=6.0 \mathrm{~Hz}, 2 \mathrm{H})$, $3.94(\mathrm{t}, J=4.9 \mathrm{~Hz}, 2 \mathrm{H}), 1.49(\mathrm{t}, J=4.8 \mathrm{~Hz}, 2 \mathrm{H}), 1.27(\mathrm{~m}, 10 \mathrm{H}), 0.81(\mathrm{t}, J=5.0 \mathrm{~Hz}, 3 \mathrm{H}), 0.09$ (s, 9H). ${ }^{13} \mathrm{C}$ NMR $\left(75 \mathrm{MHz}, \mathrm{CD}_{2} \mathrm{Cl}_{2}\right): \delta \mathrm{ppm} 156.1,141.2,140.7,139.7,138.2,137.6,132.7$, $130.2,129.2,127.7,127.6,127.4,127.3,126.6,126.4,126.2,125.6,113.6,70.2,32.2,30.1$, 29.9, 29.7, 29.6, 26.2, 23.1, 14.2. HRMS (ESI) m/z: $[\mathrm{M}+\mathrm{Na}]^{+}$Calcd for C68H80O2Sn2Na 1191.4202; Found 1191.4209. 


\section{Compound NP-1}

$\mathrm{Cu}\left(\mathrm{NO}_{3}\right)_{2} \cdot 3 \mathrm{H}_{2} \mathrm{O}(285 \mathrm{mg}, 1.18 \mathrm{mmol})$ was dissolved in $15 \mathrm{~mL}$ of anhydrous tetrahydrofuran to form blue solution. Compound 5a $(462 \mathrm{mg}, 0.54 \mathrm{mmol})$ was dissolved in $10 \mathrm{~mL}$ of anhydrous THF and added dropwise to the prepared $\mathrm{Cu}\left(\mathrm{NO}_{3}\right)_{2} \bullet 3 \mathrm{H}_{2} \mathrm{O}$ solution. The reaction mixture was stirred at room temperature for 3 days. Water was added to quench the reaction and the mixture was extracted with DCM three times. The combined organic layer was dried over anhydrous sodium sulfate. After filtration and evaporation, the crude mixture was purified with silica gel chromatography (hexane : $\mathrm{DCM}=2: 1$ ) to afford the final product as white amorphous solid (73 mg, 26\%). ${ }^{1} \mathrm{H}$ NMR (300 MHz, $\left.\mathrm{CD}_{2} \mathrm{Cl}_{2}\right): \delta \mathrm{ppm} 7.91$ (d, $\left.J=9.0 \mathrm{~Hz}, 1 \mathrm{H}\right)$, $7.29(\mathrm{~d}, J=9.0 \mathrm{~Hz}, 1 \mathrm{H}), 7.17(\mathrm{~d}, J=8.2 \mathrm{~Hz}, 2 \mathrm{H}), 6.85(\mathrm{~d}, J=8.2 \mathrm{~Hz}, 2 \mathrm{H}), 3.96(\mathrm{t}, J=6.5 \mathrm{~Hz}$, 2H), $1.52(\mathrm{t}, J=6.6 \mathrm{~Hz}, 2 \mathrm{H}), 1.33-1.06(\mathrm{~m}, 10 \mathrm{H}), 0.91-0.78(\mathrm{~m}, 3 \mathrm{H}) .{ }^{13} \mathrm{C}$ NMR $(75 \mathrm{MHz}$, $\left.\mathrm{CD}_{2} \mathrm{Cl}_{2}\right): \delta$ ppm 156.1, 138.8, 136.7, 132.6, 130.6, 126.6, 126.4, 126.1, 113.6, 70.3, 32.2, 29.9, 29.6, 29.6, 26.3, 23.1, 14.3. HRMS (APCI) m/z: $[\mathrm{M}+\mathrm{Na}]^{+}$Calcd for $\mathrm{C} 76 \mathrm{H} 93 \mathrm{O} 4 \mathrm{Na}$ 1092.7068; Found 1092.7064.

\section{Compound NP-2}

Compound (NP-2) (131 mg, white amorphous solid, 37\%) was prepared according to the similar procedures as compound (NP-1). ${ }^{1} \mathrm{H}$ NMR (300 MHz, $\left.\mathrm{CDCl}_{3}\right): \delta 7.92(\mathrm{~d}, J=9.0 \mathrm{~Hz}$, 1H), $7.32(\mathrm{~s}, 4 \mathrm{H}), 7.30(\mathrm{~d}, J=9.1 \mathrm{~Hz}, 1 \mathrm{H}), 7.19(\mathrm{~d}, J=8.2 \mathrm{~Hz}, 2 \mathrm{H}), 6.91(\mathrm{~d}, J=8.2 \mathrm{~Hz}, 2 \mathrm{H})$, $3.96(\mathrm{t}, J=6.5 \mathrm{~Hz}, 2 \mathrm{H}), 1.50(\mathrm{~s}, 2 \mathrm{H}), 1.16(\mathrm{~d}, J=3.8 \mathrm{~Hz}, 10 \mathrm{H}), 0.82(\mathrm{t}, J=6.9 \mathrm{~Hz}, 3 \mathrm{H}) .{ }^{13} \mathrm{C}$ NMR (75 MHz, $\mathrm{CD}_{2} \mathrm{Cl}_{2}$ ): $\delta$ ppm 155.0, 139.6, 138.5, 128.4, 126.9, 126.7, 126.4, 125.3, 69.7, 31.8, 29.9, 29.4, 29.2, 29.2, 25.8, 22.6, 13.8. HRMS (APCI) m/z: $[\mathrm{M}+\mathrm{Na}]^{+}$Calcd for C100H106O4Na 1395.8242; Found 1395.8241.

\section{Compound NP-3}

Compound NP-3 was synthesized according to the similar procedures as compounds NP-1 and NP-2. The peak of compound NP-3 can be unambiguously identified in the MALDI-TOF mass spectrum from the reaction mixture $\left(\mathrm{m} / \mathrm{z}\right.$ : $[\mathrm{M}+\mathrm{Na}]^{+} \mathrm{Calcd}$ for $\mathrm{C}_{124} \mathrm{H}_{124} \mathrm{O}_{4} \mathrm{Na}$ 1699.9500; Found 1699.3920). However, due to the poor solubility of compound NP-3 and its low yield, the reaction mixture is very difficult to purify to give the pure final product. 


\section{Fluorescence spectra of NP-1 and NP-2}

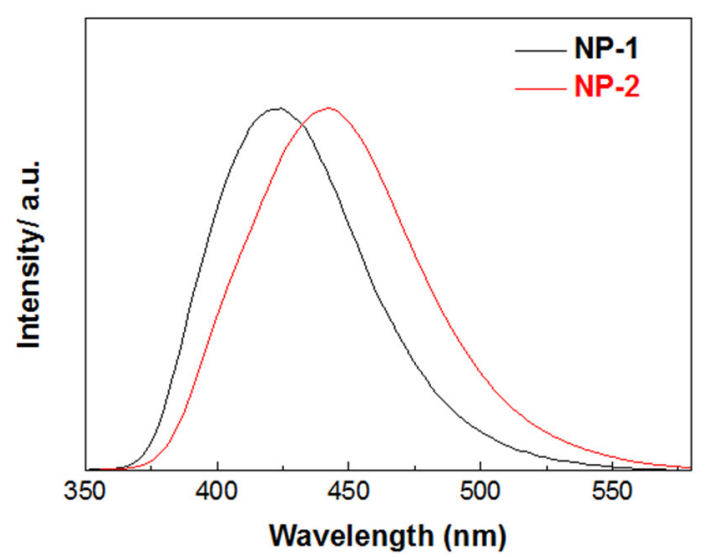

Figure S1. Normalized fluorescence spectra of NP-1 and NP-2 in DCM (excitation wavelength at the absorption maximum).

\section{DFT calculations}

Theoretical calculations were performed with the Gaussian09 program suite. ${ }^{3}$ All calculations were carried out using the density functional theory (DFT) method with Becke's three-parameter hybrid exchange functionals and the Lee-Yang-Parr correlation functional (B3LYP) employing the 6-31G(d,p) basis set for all atoms. ${ }^{4}$ NOON calcualtions were and the diradical character is defined as the occupation number of the lowest unoccupied natural orbital (LUNO). Time-dependent DFT (TD-DFT) calculations have been performed at the B3LYP/6$31 \mathrm{G}^{*}$ level of theory ${ }^{5}$.

NP-1
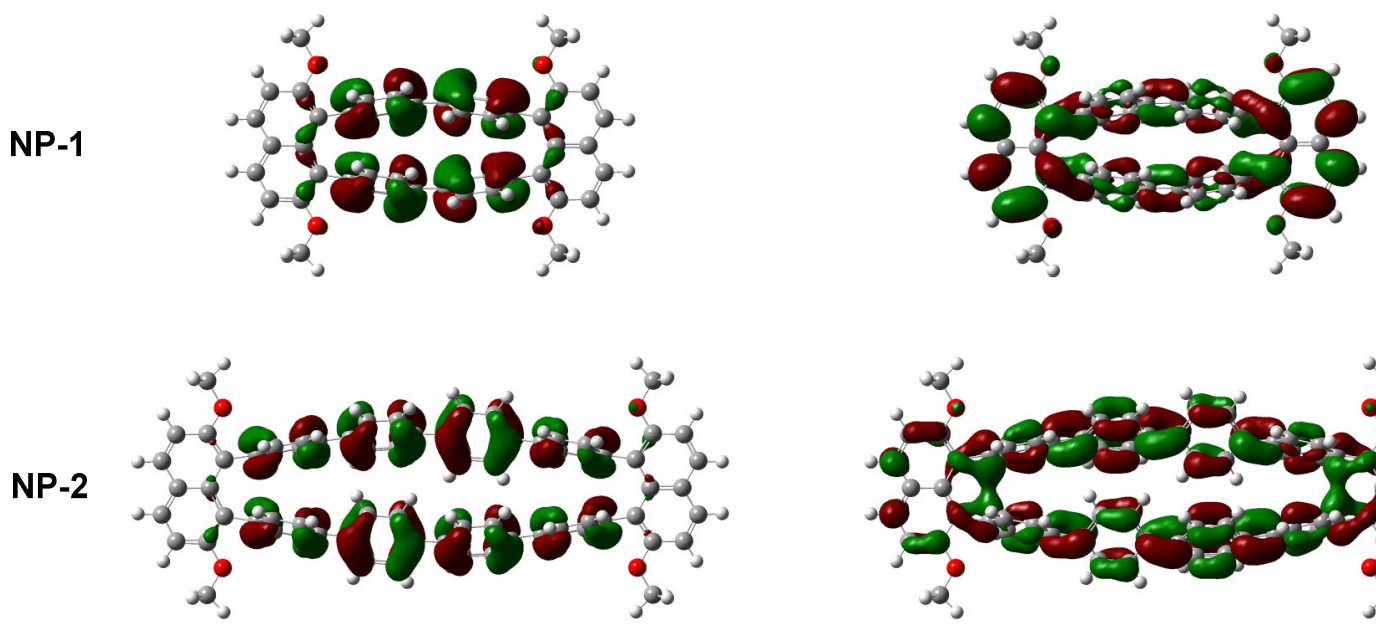

HOMO

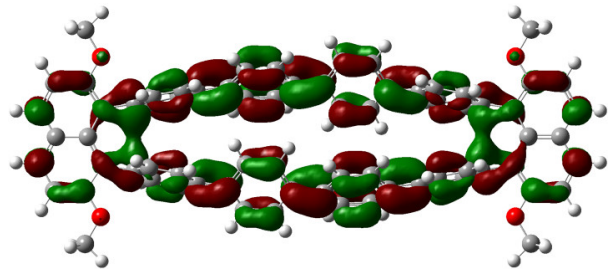

LUMO

Figure S2. Calculated (RB3LYP/6-31G*) HOMO and LUMO profiles of NP-1 and NP-2. 


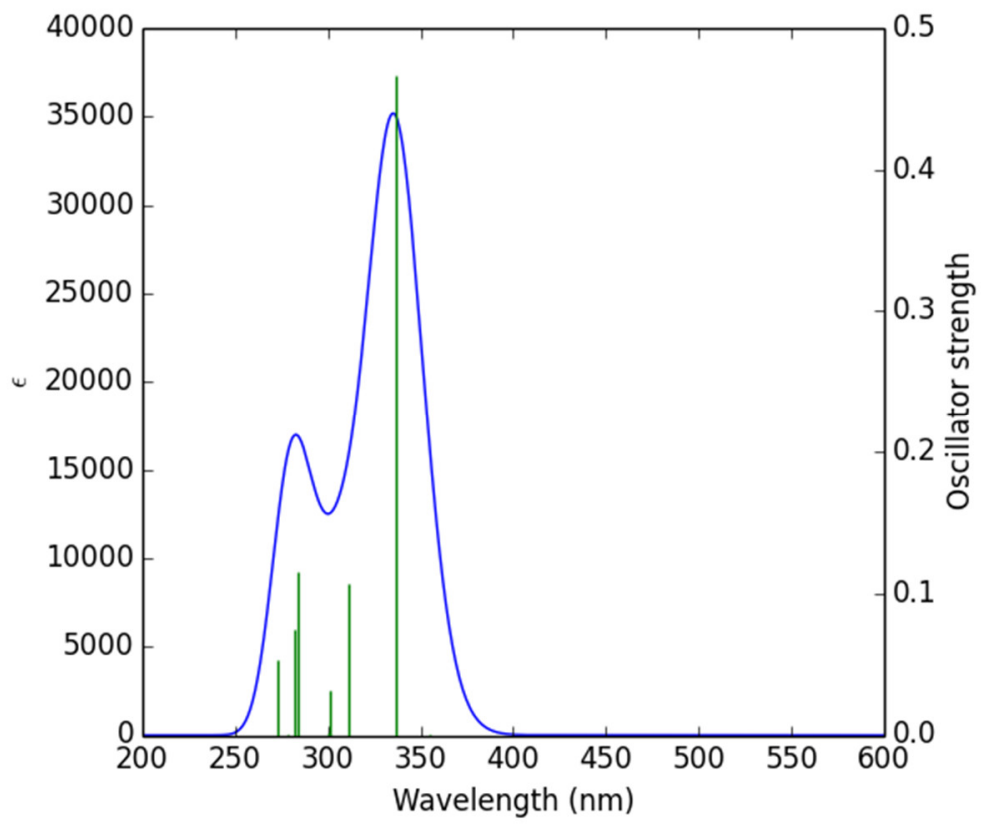

Figure S3. Calculated (RB3LYP/6-31G*) absorption spectrum of NP-1.

Table S1. Selected TD-DFT (B3LYP/6-31G(d,p)) calculated energies, oscillator strength and compositions of major electronic transitions of NP-1.

\begin{tabular}{|l|l|l|}
\hline Wavelength $(\mathrm{nm})$ & Osc. Strength $(f)$ & Major Contribution \\
\hline 336.6 & 0.4661 & $\begin{array}{l}\text { H-1->LUMO }(89 \%) \text { H-2->L+1 (6\%), H-1- } \\
>\mathrm{L}+2\end{array}$ \\
\hline 311.0 & 0.1071 & $\begin{array}{l}\text { H-2->L+1 (83\%)H-1->LUMO (4\%), H-1- } \\
>\text { L }+2\end{array}$ \\
\hline 284.1 & 0.1154 & H-5->LUMO (40\%), HOMO->L+3 (53\%) \\
\hline
\end{tabular}




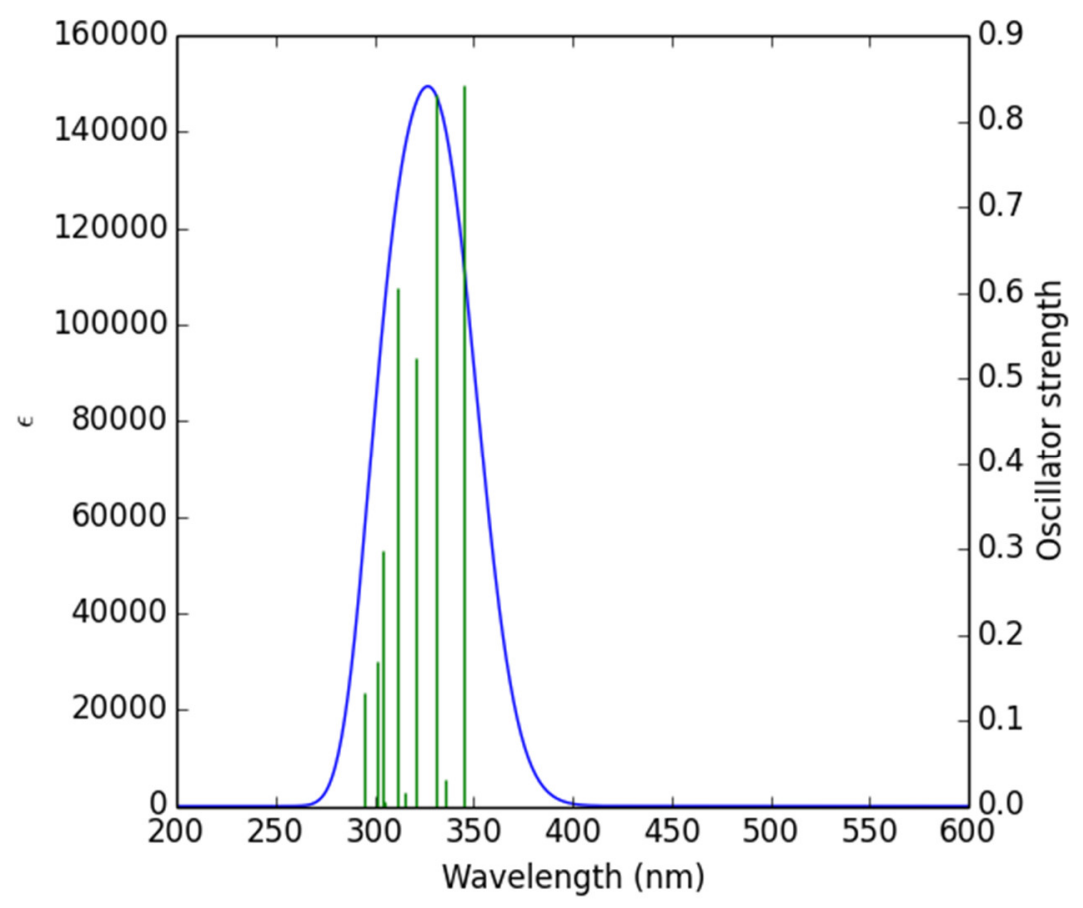

Figure S4. Calculated (RB3LYP/6-31G*) absorption spectrum of NP-2.

Table S2. Selected TD-DFT (B3LYP/6-31G(d,p)) calculated energies, oscillator strength and compositions of major electronic transitions of NP-2.

\begin{tabular}{|l|l|l|}
\hline Wavelength $(\mathrm{nm})$ & Osc. Strength $(f)$ & Major Contribution \\
\hline 345.0 & 0.8423 & H-1->LUMO (87\%) H-2->L+1 (9\%) \\
\hline 331.3 & 0.8305 & $\begin{array}{l}\text { HOMO->L+2 (80\%) H-3->LUMO (9\%), } \\
\text { H-2->L+1 }\end{array}$ \\
\hline 320.5 & 0.5245 & $\begin{array}{l}\text { H-3->LUMO (46\%), H-2->L+2 (11\%), H- } \\
1->\text { L+3 }\end{array}$ \\
\hline 311.8 & 0.6057 & $\begin{array}{l}\text { H-3->LUMO (36\%), H-2->L+1 (15\%), H- } \\
\text { 2->L+2 }\end{array}$ \\
\hline 303.6 & 0.2977 & $\begin{array}{l}\text { H-4->LUMO (28\%), H-2->L+1 (10\%), H- } \\
1->\text { L+3 }\end{array}$ \\
\hline
\end{tabular}




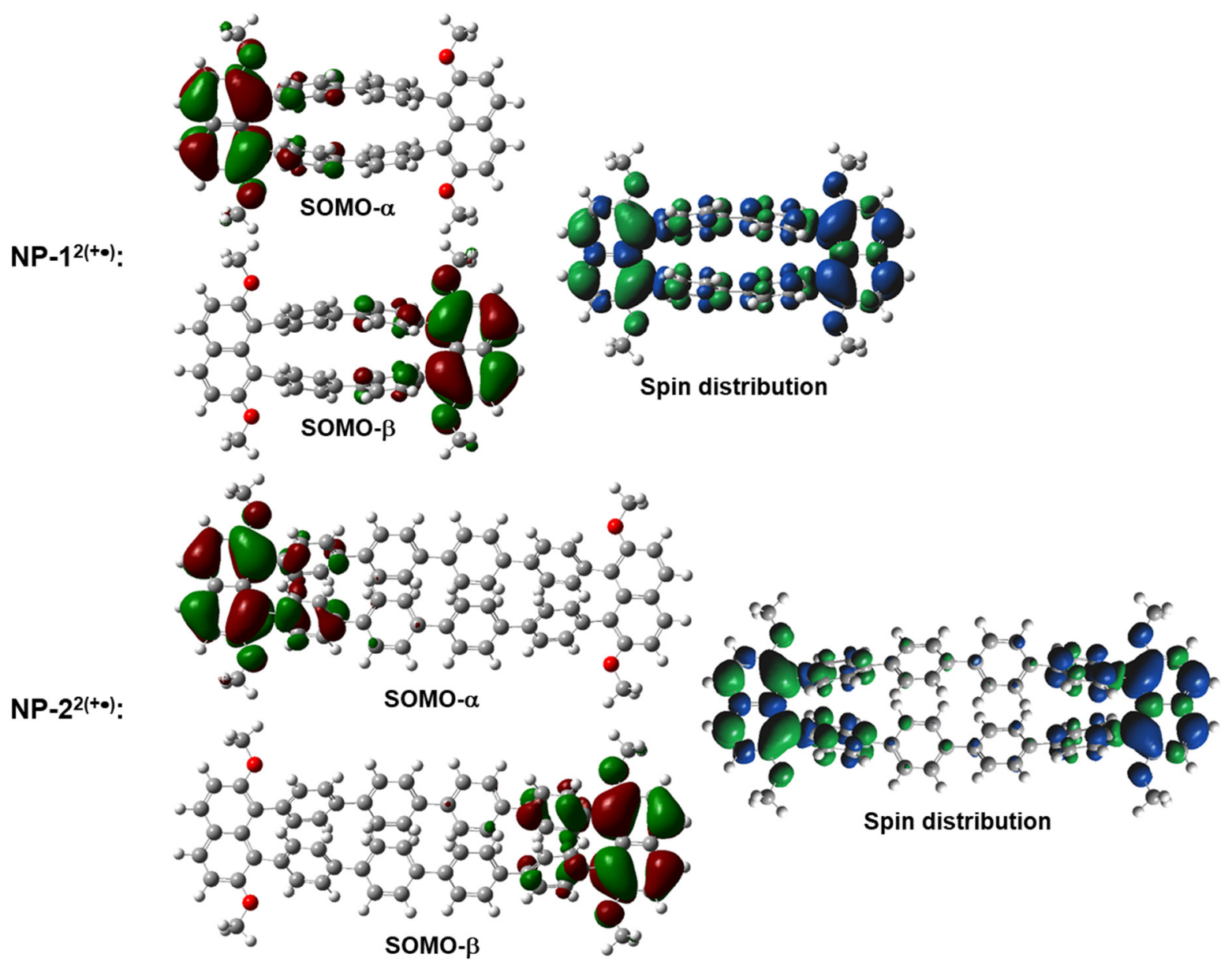

Figure S5. Calculated (UCAM-B3LYP/6-31G(d,p)) molecular orbital profiles of $\alpha$ and $\beta$ spins

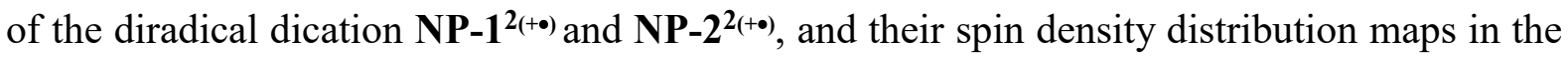
singlet biradical state.

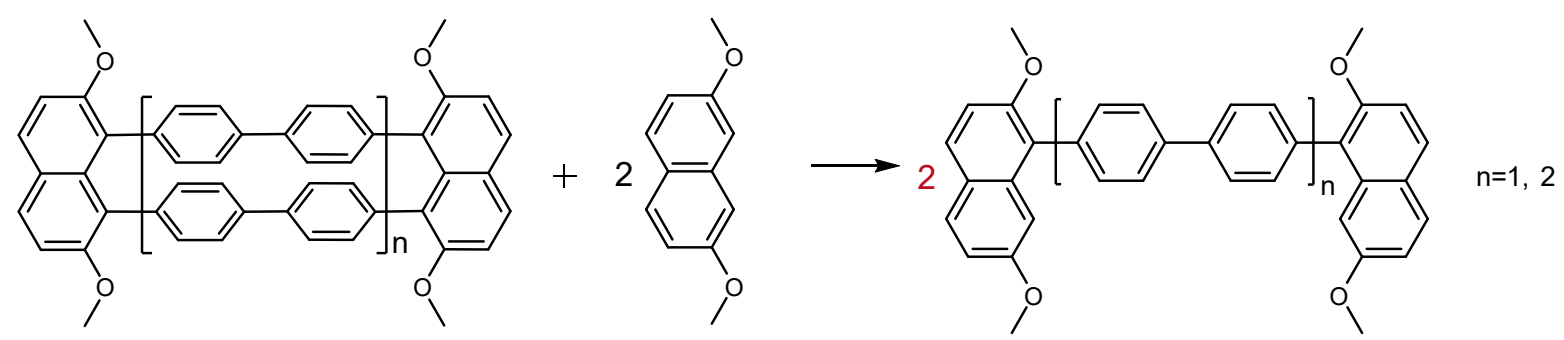

Figure S6. Homodesmotic reactions used for the strain energy calculation for NP-1 and NP-2. In order to simplify the computational process, four octyloxy side chains are replaced with four methyloxy groups. 


\section{X-ray Crystallographic Data of NP-1, NP-2 and 3c.}

The X-ray intensity data were measured at low temperature $(\mathrm{T}=100 \mathrm{~K})$, using a four circles goniometer Kappa geometry, Bruker AXS D8 Venture, equipped with a Photon 100 CMOS active pixel sensor detector. A Copper monochromatized (= $1.54178 \AA$ ) X-Ray radiation was used. Frames were integrated with the Bruker SAINT ${ }^{6}$ software package. Data were corrected for absorption effects using the multi-scan method (SADABS). ${ }^{7}$ Molecule was solved with the software SHELXT, ${ }^{8}$ using a Dual Space method. Refinement of the structure was performed by least squares procedures on weighted F2 values using the SHELXL-version 2014/6 ${ }^{9}$ included in the WinGx system programs for Windows. ${ }^{10}$ Singlet crystal of compound NP-1, NP-2 and 3c were all obtained through slow diffusion of methanol to their DCM solutions.

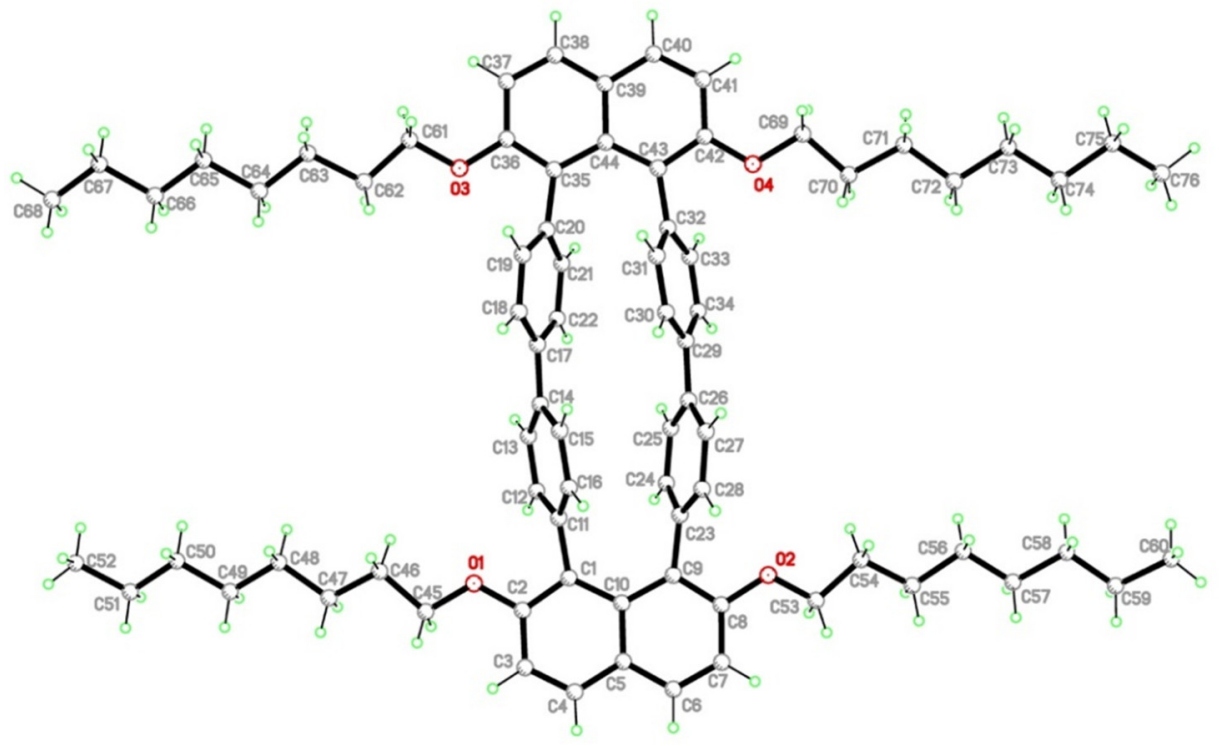

Table S3. Crystallographic data and structure refinement for NP-1, displacement ellipsoids are drawn at the $30 \%$ probability level.

$\begin{array}{ll}\text { Identification code } & \mathrm{NP}-1 \\ \text { Chemical formula } & \mathrm{C}_{76} \mathrm{H}_{92} \mathrm{O}_{4} \\ \text { Formula weight } & 1069.49 \mathrm{~g} / \mathrm{mol} \\ \text { Temperature } & 100(2) \mathrm{K} \\ \text { Wavelength } & 0.71073 \AA \\ \text { Crystal size } & 0.255 \times 0.382 \times 0.523 \mathrm{~mm} \\ \text { Crystal system } & \text { triclinic } \\ \text { Unit cell dimensions } & \mathrm{a}=12.8452(6) \AA \quad \mathrm{S} \quad \alpha=73.506(2)^{\circ}\end{array}$




$\begin{array}{lll} & \mathrm{b}=13.5276(6) \AA & \beta=83.683(2)^{\circ} \\ & \mathrm{c}=18.7445(8) \AA & \gamma=83.257(2)^{\circ} \\ \text { Volume } & 3091.4(2) \AA^{3} & \\ \text { Z } & 2 \\ \text { Density (calculated) } & 1.149 \mathrm{~g} / \mathrm{cm}^{3} & \\ \text { Absorption coefficient } & 0.069 \mathrm{~mm}^{-1} & \\ \mathrm{~F}(000) & 1160\end{array}$

Table S4. Crystallographic data and structure refinement for NP-1.

\begin{tabular}{|c|c|}
\hline Theta range for data collection & 1.58 to $28.28^{\circ}$ \\
\hline Index ranges & $-17<=\mathrm{h}<=17,-18<=\mathrm{k}<=18,-24<=1<=24$ \\
\hline Reflections collected & 85072 \\
\hline Independent reflections & $15322[\mathrm{R}(\mathrm{int})=0.0298]$ \\
\hline $\begin{array}{l}\text { Coverage of independent } \\
\text { reflections }\end{array}$ & $100.0 \%$ \\
\hline Absorption correction & Multi-Scan \\
\hline Max. and min. transmission & 0.7461 and 0.7242 \\
\hline Structure solution technique & direct methods \\
\hline Structure solution program & SHELXS-97 (Sheldrick 2008) \\
\hline Refinement method & Full-matrix least-squares on $\mathrm{F}^{2}$ \\
\hline Refinement program & SHELXL-2014/7 (Sheldrick, 2014) \\
\hline Function minimized & $\Sigma \mathrm{w}\left(\mathrm{F}_{\mathrm{o}}^{2}-\mathrm{F}_{\mathrm{c}}^{2}\right)^{2}$ \\
\hline Data / restraints / parameters & $15322 / 0 / 725$ \\
\hline Goodness-of-fit on $\mathrm{F}^{2}$ & 1.075 \\
\hline$\Delta / \sigma_{\max }$ & 0.001 \\
\hline \multirow[t]{2}{*}{ Final $\mathrm{R}$ indices } & 12607 data; $\mathrm{I}>2 \sigma(\mathrm{I}) \mathrm{R} 1=0.0421, \mathrm{wR} 2=0.1208$ \\
\hline & $\mathrm{R} 1=0.0545, \mathrm{wR} 2=0.1340$ \\
\hline Weighting scheme & $\begin{array}{l}\mathrm{w}=1 /\left[\sigma^{2}\left(\mathrm{~F}_{\mathrm{o}}^{2}\right)+(0.0716 \mathrm{P})^{2}+0.8027 \mathrm{P}\right] \\
\text { where } \mathrm{P}=\left(\mathrm{F}_{\mathrm{o}}^{2}+2 \mathrm{~F}_{\mathrm{c}}^{2}\right) / 3\end{array}$ \\
\hline Largest diff. peak and hole & 0.443 and -0.365 e $\AA^{-3}$ \\
\hline R.M.S. deviation from mean & $0.090 \mathrm{e}^{-3}$ \\
\hline
\end{tabular}




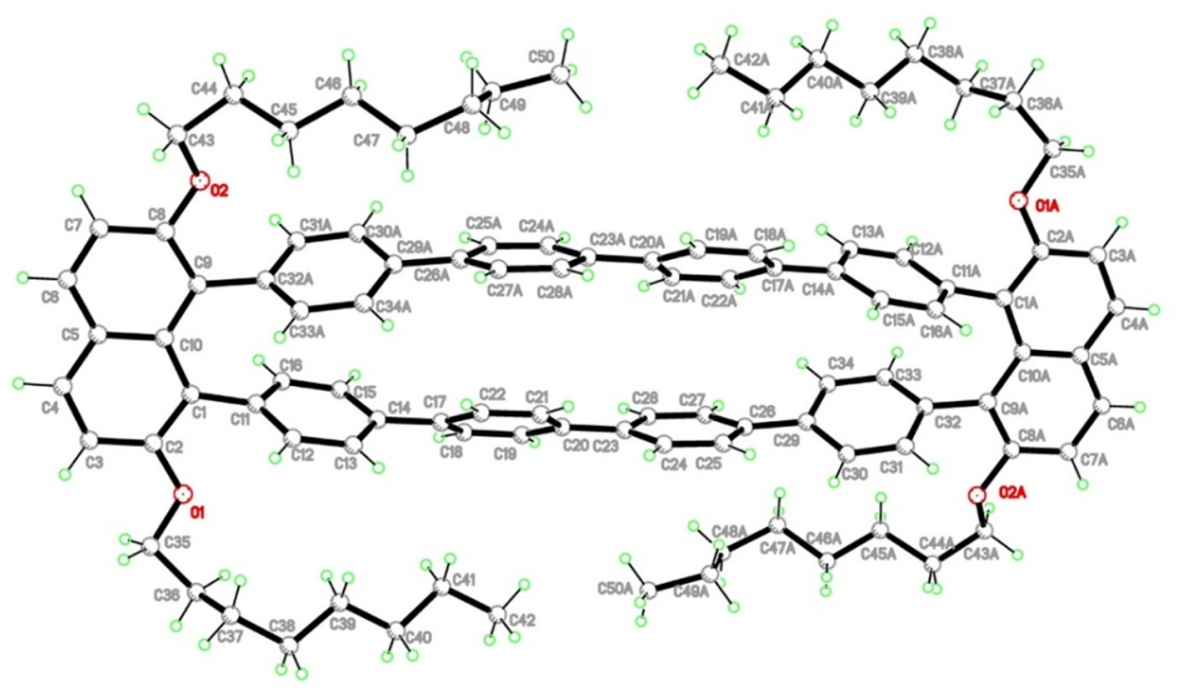

Table S5. Crystallographic data and structure refinement for NP-2, displacement ellipsoids are drawn at the $30 \%$ probability level.

$\begin{array}{lll}\text { Identification code } & \mathrm{NP}-2 \\ \text { Chemical formula } & \mathrm{C}_{100} \mathrm{H}_{108} \mathrm{O}_{4} & \\ \text { Formula weight } & 1373.86 \mathrm{~g} / \mathrm{mol} & \\ \text { Temperature } & 170(2) \mathrm{K} & \\ \text { Wavelength } & 1.54178 \AA & \\ \text { Crystal size } & 0.044 \times 0.049 \times 0.259 \mathrm{~mm} & \\ \text { Crystal system } & \text { monoclinic } & \\ \text { Space group } & \mathrm{P} 121 / \mathrm{c} 1 & \alpha=90^{\circ} \\ \text { Unit cell dimensions } & \mathrm{a}=14.6696(7) \AA & \beta=92.809(3)^{\circ} \\ & \mathrm{b}=7.6483(3) \AA & \gamma=90^{\circ} \\ & \mathrm{c}=34.7642(12) \AA & \\ \text { Volume } & 3895.8(3) \AA^{3} & \\ Z & 2 \\ \text { Density (calculated) } & 1.171 \mathrm{~g} / \mathrm{cm}^{3} & \\ \text { Absorption coefficient } & 0.528 \mathrm{~mm}^{-1} & \\ \text { F(000) } & 1480\end{array}$

Table S6. Crystallographic data and structure refinement for NP-2.

$\begin{array}{ll}\text { Theta range for data collection } & 2.54 \text { to } 66.48^{\circ} \\ \text { Index ranges } & -17<=\mathrm{h}<=17,-8<=\mathrm{k}<=9,-41<=\mathrm{l}<=38 \\ \text { Reflections collected } & 22903 \\ \text { Independent reflections } & 6811[\mathrm{R}(\mathrm{int})=0.0960]\end{array}$




$\begin{array}{ll}\begin{array}{l}\text { Coverage of independent } \\ \text { reflections }\end{array} & 99.1 \% \\ \text { Absorption correction } & \text { Multi-Scan } \\ \text { Max. and min. transmission } & 0.7528 \text { and } 0.6343 \\ \text { Structure solution technique } & \text { direct methods } \\ \text { Structure solution program } & \text { SHELXS-97 (Sheldrick 2008) } \\ \text { Refinement method } & \text { Full-matrix least-squares on } \mathrm{F}^{2} \\ \text { Refinement program } & \text { SHELXL-2014/7 (Sheldrick, 2014) } \\ \text { Function minimized } & \Sigma \mathrm{w}\left(\mathrm{F}_{\mathrm{o}}^{2}-\mathrm{F}_{\mathrm{c}}^{2}\right)^{2} \\ \text { Data / restraints / parameters } & 6811 / 0 / 471 \\ \text { Goodness-of-fit on } \mathrm{F}^{2} & 1.009 \\ \text { Final R indices } & 4200 \mathrm{data} ; \mathrm{I}>2 \sigma(\mathrm{I}) \mathrm{R} 1=0.0592, \mathrm{wR} 2=0.1286 \\ & \text { all data } \\ \text { Largest diff. peak and hole } & \mathrm{w}=1 /\left[\sigma^{2}\left(\mathrm{~F}_{\mathrm{o}}{ }^{2}\right)+(0.0640 \mathrm{P})^{2}+0.5924 \mathrm{P}\right] \\ \text { R.M.S. deviation from mean } & \text { where } \mathrm{P}=\left(\mathrm{F}_{\mathrm{o}}^{2}+2 \mathrm{~F}_{\mathrm{c}}^{2}\right) / 3 \\ \text { Weighting scheme } & 0.229 \text { and }-0.256 \mathrm{e} \AA^{-3} \\ & \end{array}$

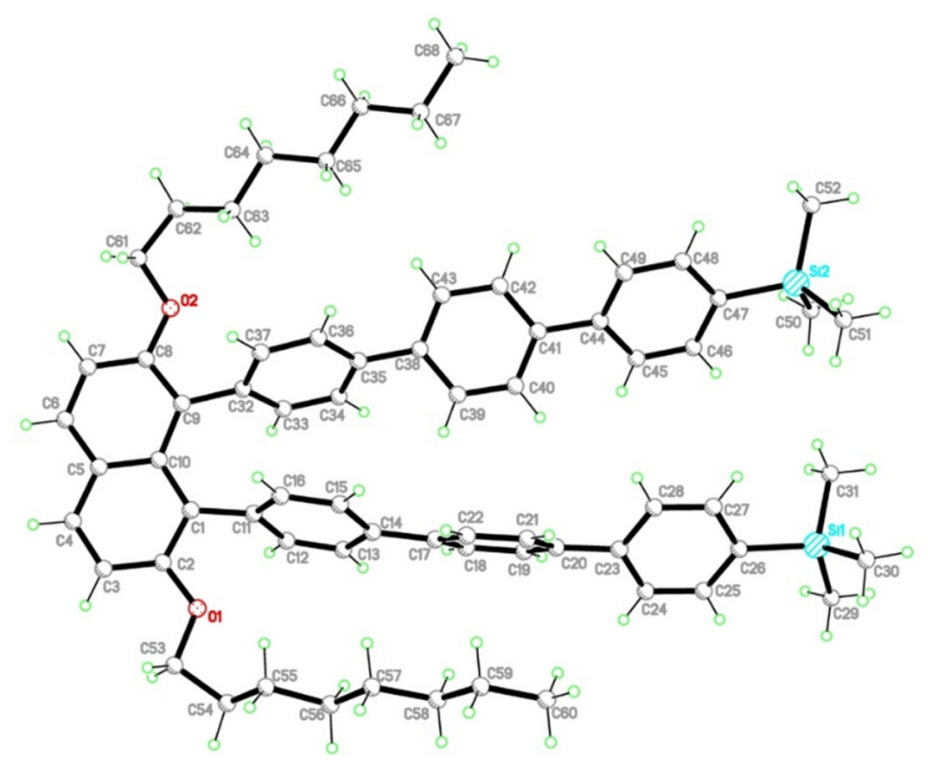

Table S7. Crystallographic data and structure refinement for $\mathbf{3 c}$, displacement ellipsoids are drawn at the $30 \%$ probability level.

$\begin{array}{ll}\text { Identification code } & 3 \mathrm{c} \\ \text { Chemical formula } & \mathrm{C}_{68} \mathrm{H}_{80} \mathrm{O}_{2} \mathrm{Si}_{2} \\ \text { Formula weight } & 985.50 \mathrm{~g} / \mathrm{mol} \\ \text { Temperature } & 100(2) \mathrm{K}\end{array}$




$\begin{array}{lll}\text { Wavelength } & 1.54178 \AA \\ \text { Crystal size } & 0.074 \times 0.111 \times 0.430 \mathrm{~mm} \\ \text { Crystal system } & \text { monoclinic } & \\ \text { Space group } & \mathrm{P} 121 / \mathrm{c} 1 & \alpha=90^{\circ} \\ \text { Unit cell dimensions } & \mathrm{a}=21.2655(6) \AA & \beta=105.1611(10)^{\circ} \\ & \mathrm{b}=6.8120(2) \AA & \gamma=90^{\circ} \\ \text { Volume } & \mathrm{c}=40.4696(12) \AA & \\ \text { Z } & 5658.4(3) \AA^{3} & \\ \text { Density (calculated) } & 4 & \\ \text { Absorption coefficient } & 0.899 \mathrm{~mm}^{-1} & \\ \text { F(000) } & 2128 & \end{array}$

Table S8. Crystallographic data and structure refinement for $\mathbf{3 c}$.

\begin{tabular}{|c|c|}
\hline Theta range for data collection & 2.15 to $66.54^{\circ}$ \\
\hline Index ranges & $-25<=\mathrm{h}<=25,-8<=\mathrm{k}<=8,-46<=1<=48$ \\
\hline Reflections collected & 46664 \\
\hline Independent reflections & $9903[\mathrm{R}(\mathrm{int})=0.0550]$ \\
\hline $\begin{array}{l}\text { Coverage of independent } \\
\text { reflections }\end{array}$ & t $99.4 \%$ \\
\hline Absorption correction & Multi-Scan \\
\hline Max. and min. transmission & 0.9360 and 0.6990 \\
\hline Structure solution technique & direct methods \\
\hline Structure solution program & SHELXT-(Sheldrick 2015) \\
\hline Refinement method & Full-matrix least-squares on $\mathrm{F}^{2}$ \\
\hline Refinement program & SHELXL-2014/7 (Sheldrick, 2014) \\
\hline Function minimized & $\Sigma \mathrm{w}\left(\mathrm{F}_{\mathrm{o}}^{2}-\mathrm{F}_{\mathrm{c}}^{2}\right)^{2}$ \\
\hline Data / restraints / parameters & $9903 / 0 / 657$ \\
\hline Goodness-of-fit on $\mathrm{F}^{2}$ & 1.033 \\
\hline \multirow[t]{2}{*}{ Final $\mathrm{R}$ indices } & 8659 data; $\mathrm{I}>2 \sigma(\mathrm{I}) \mathrm{R} 1=0.0445, \mathrm{wR} 2=0.1141$ \\
\hline & $\mathrm{R} 1=0.0516, \mathrm{wR} 2=0.1195$ \\
\hline \multirow{2}{*}{ Weighting scheme } & $\mathrm{W}=1 /\left[\sigma^{2}\left(\mathrm{~F}_{\mathrm{o}}^{2}\right)+(0.0617 \mathrm{P})^{2}+2.8824 \mathrm{P}\right]$ \\
\hline & where $\mathrm{P}=\left(\mathrm{F}_{\mathrm{o}}^{2}+2 \mathrm{~F}_{\mathrm{c}}^{2}\right) / 3$ \\
\hline Largest diff. peak and hole & 0.392 and $-0.412 \mathrm{e}^{-3}$ \\
\hline R.M.S. deviation from mean & $0.060 \mathrm{e} \AA^{-3}$ \\
\hline
\end{tabular}



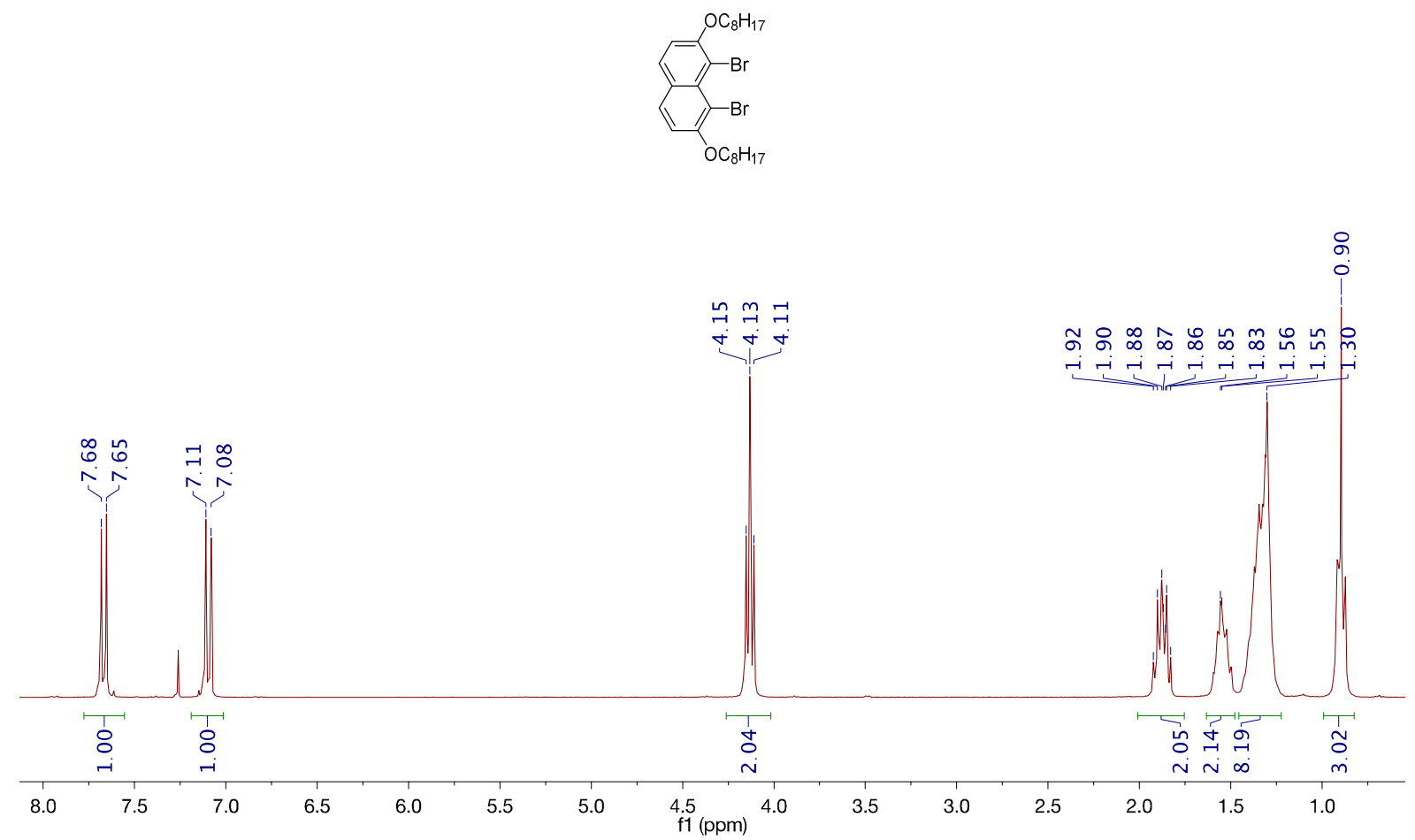

Figure S7. ${ }^{1} \mathrm{H}$ NMR spectrum of compound $1\left(300 \mathrm{MHz}, \mathrm{CDCl}_{3}, \mathrm{rt}\right)$
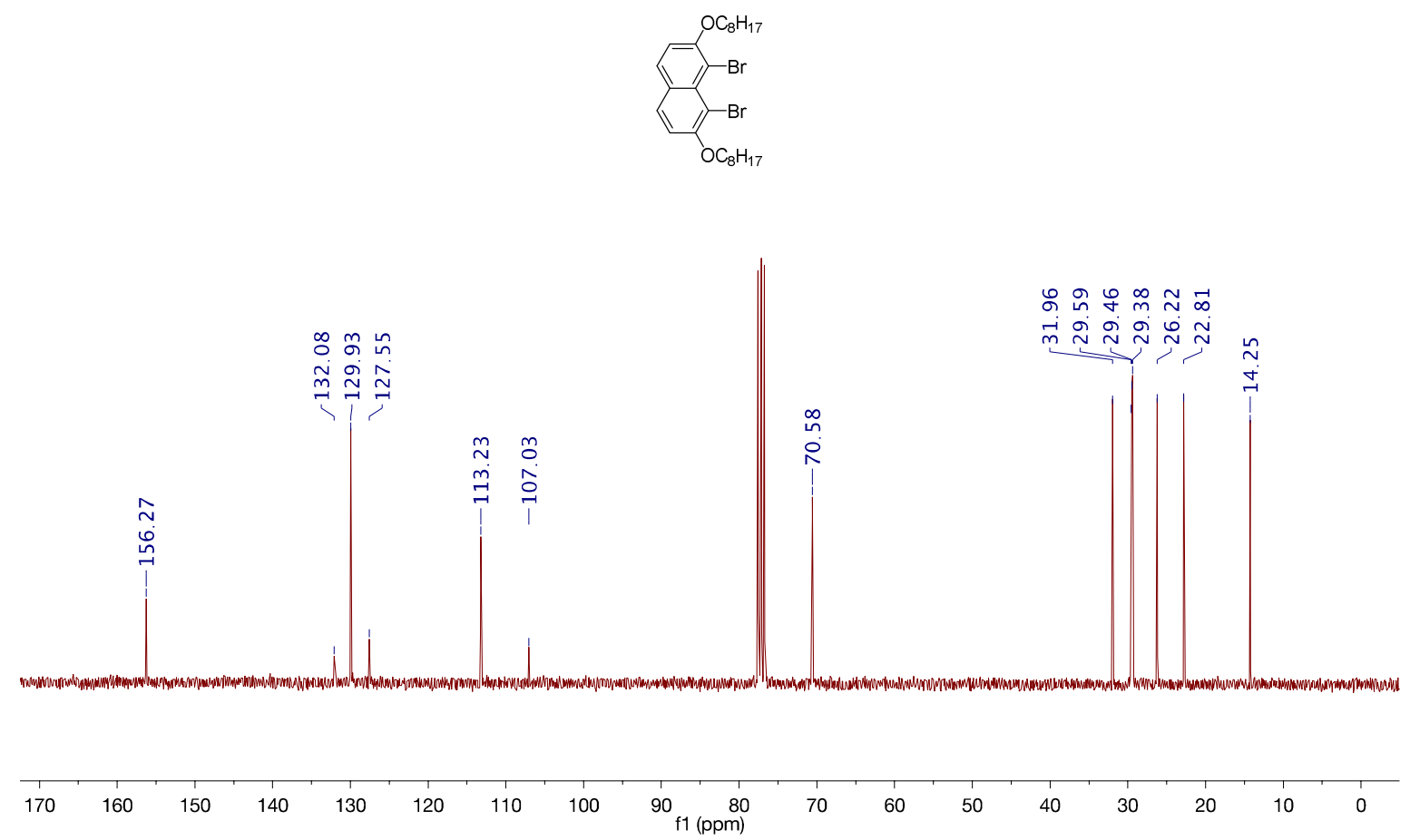

Figure S8. ${ }^{13} \mathrm{C}$ NMR spectrum of compound 1 (75 $\mathrm{MHz}^{\mathrm{CDCl}} \mathrm{CD}_{3}, \mathrm{rt}$ ) 

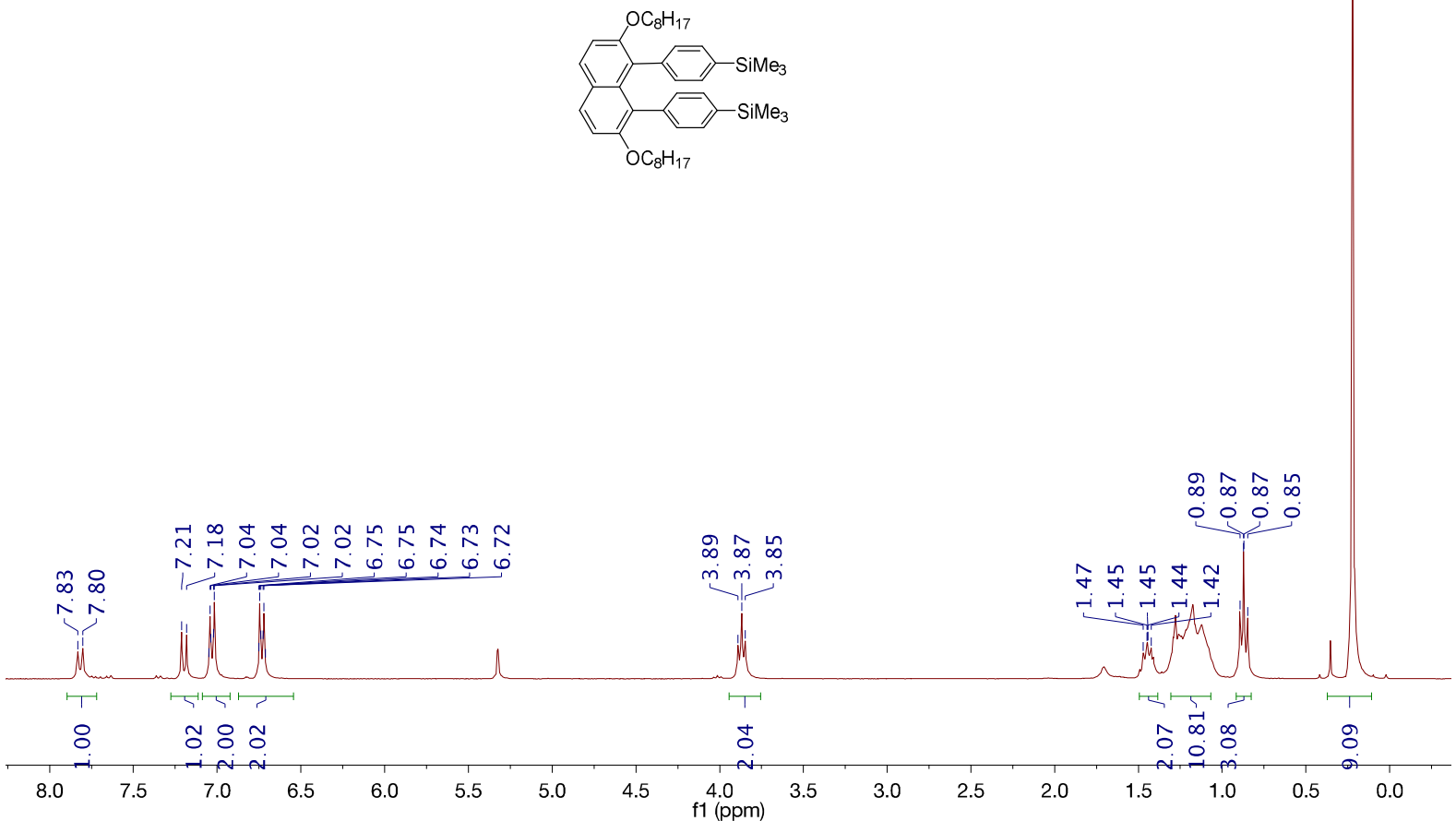

Figure S9. ${ }^{1} \mathrm{H}$ NMR spectrum of compound 3a $\left(300 \mathrm{MHz}, \mathrm{CD}_{2} \mathrm{Cl}_{2}, \mathrm{rt}\right)$

1phenyl SiMe3-13C
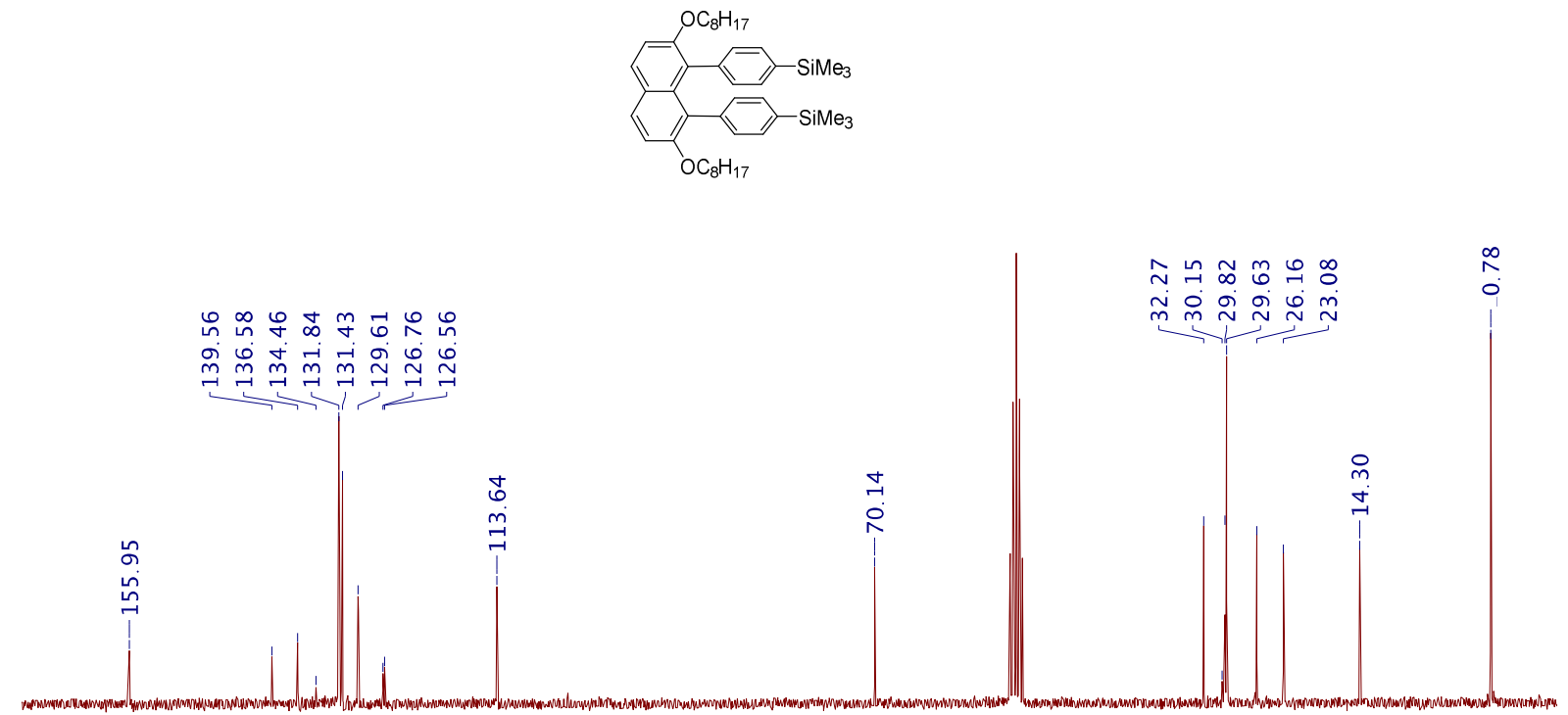

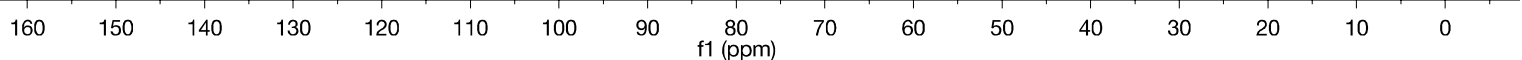

Figure S10. ${ }^{13} \mathrm{C}$ NMR spectrum of compound 3a $\left(75 \mathrm{MHz}, \mathrm{CD}_{2} \mathrm{Cl}_{2}\right.$, rt) 

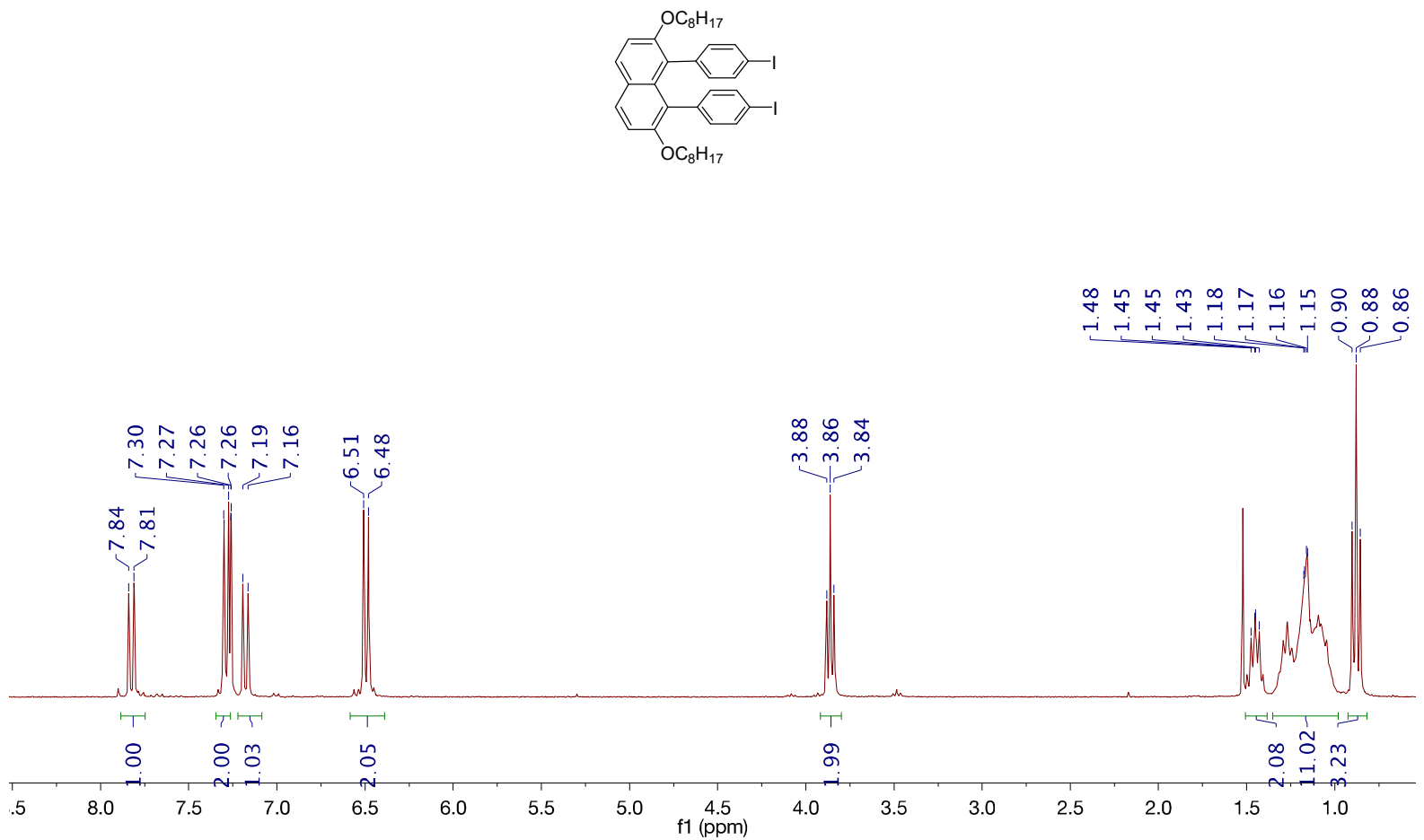

Figure S11. ${ }^{1} \mathrm{H}$ NMR spectrum of compound $4 \mathbf{a}\left(300 \mathrm{MHz}, \mathrm{CDCl}_{3}\right.$, rt)

13C 1phenyl-I CDCl3

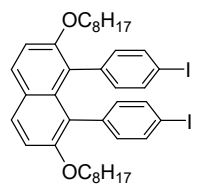

$\stackrel{\infty}{\wedge} \infty$ in

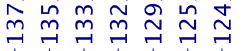

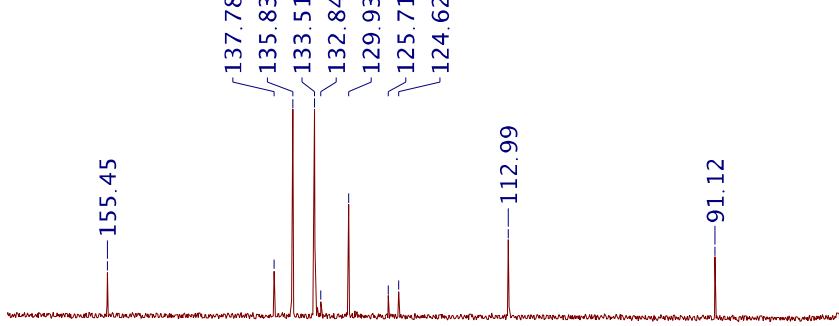

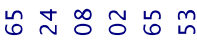

ก่

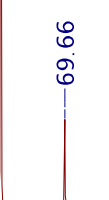

$\stackrel{\text { m. }}{\rightarrow}$

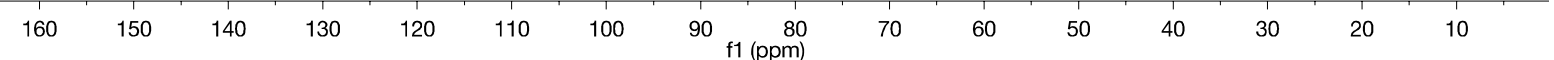

Figure S12. ${ }^{13} \mathrm{C}$ NMR spectrum of compound $4 \mathbf{a}\left(75 \mathrm{MHz}, \mathrm{CDCl}_{3}, \mathrm{rt}\right)$ 


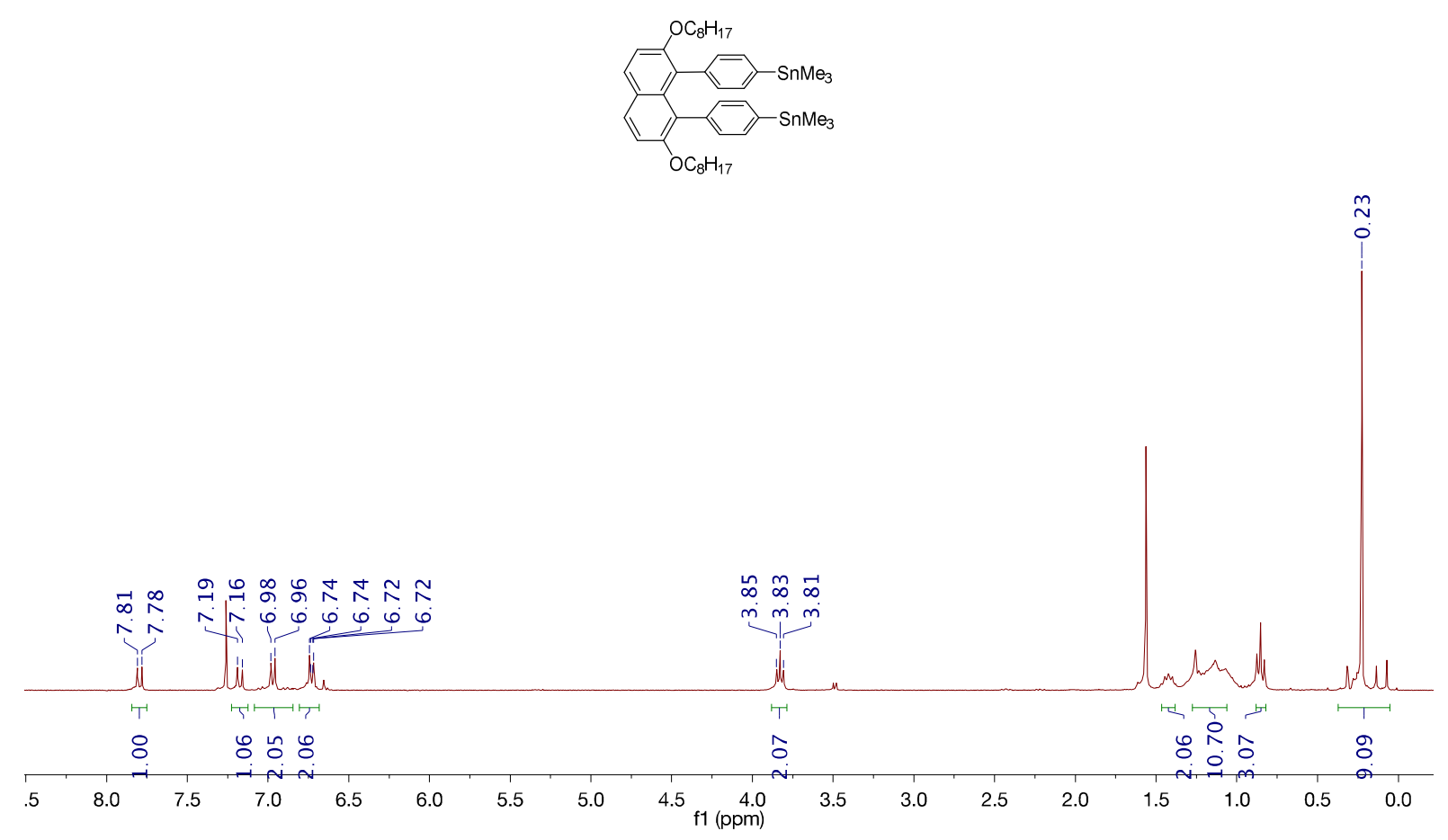

Figure S13. ${ }^{1} \mathrm{H}$ NMR spectrum of compound 5a (300 $\mathrm{MHz}, \mathrm{CDCl}_{3}$, rt)

1 Phenyl-SnMe3-13C
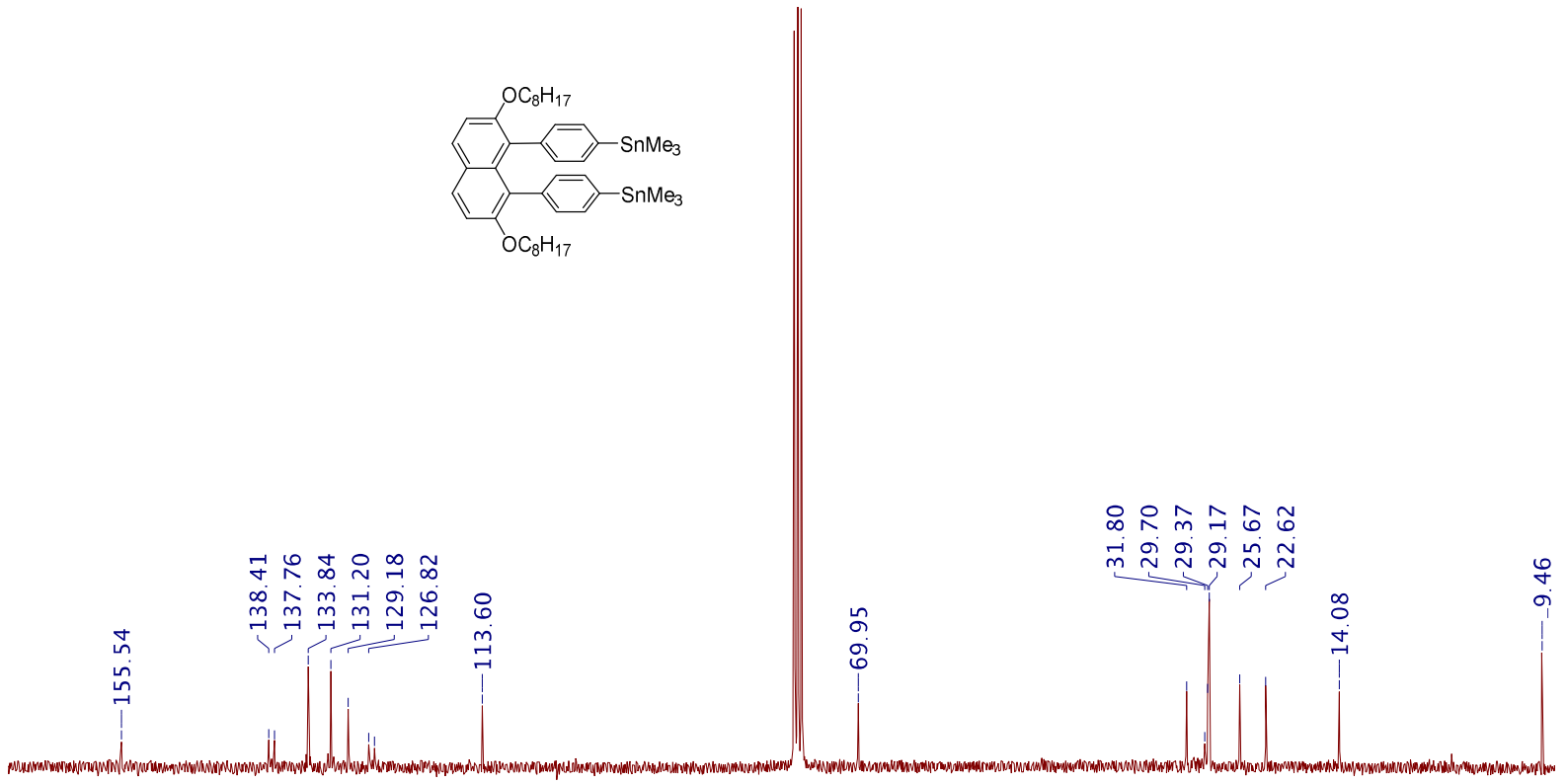

오숭승

ஸे

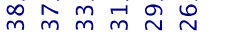

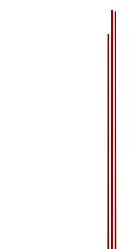

Figure S14. ${ }^{13} \mathrm{C}$ NMR spectrum of compound $5 \mathbf{a}\left(75 \mathrm{MHz}^{\mathrm{CDCl}} 3\right.$, rt) 

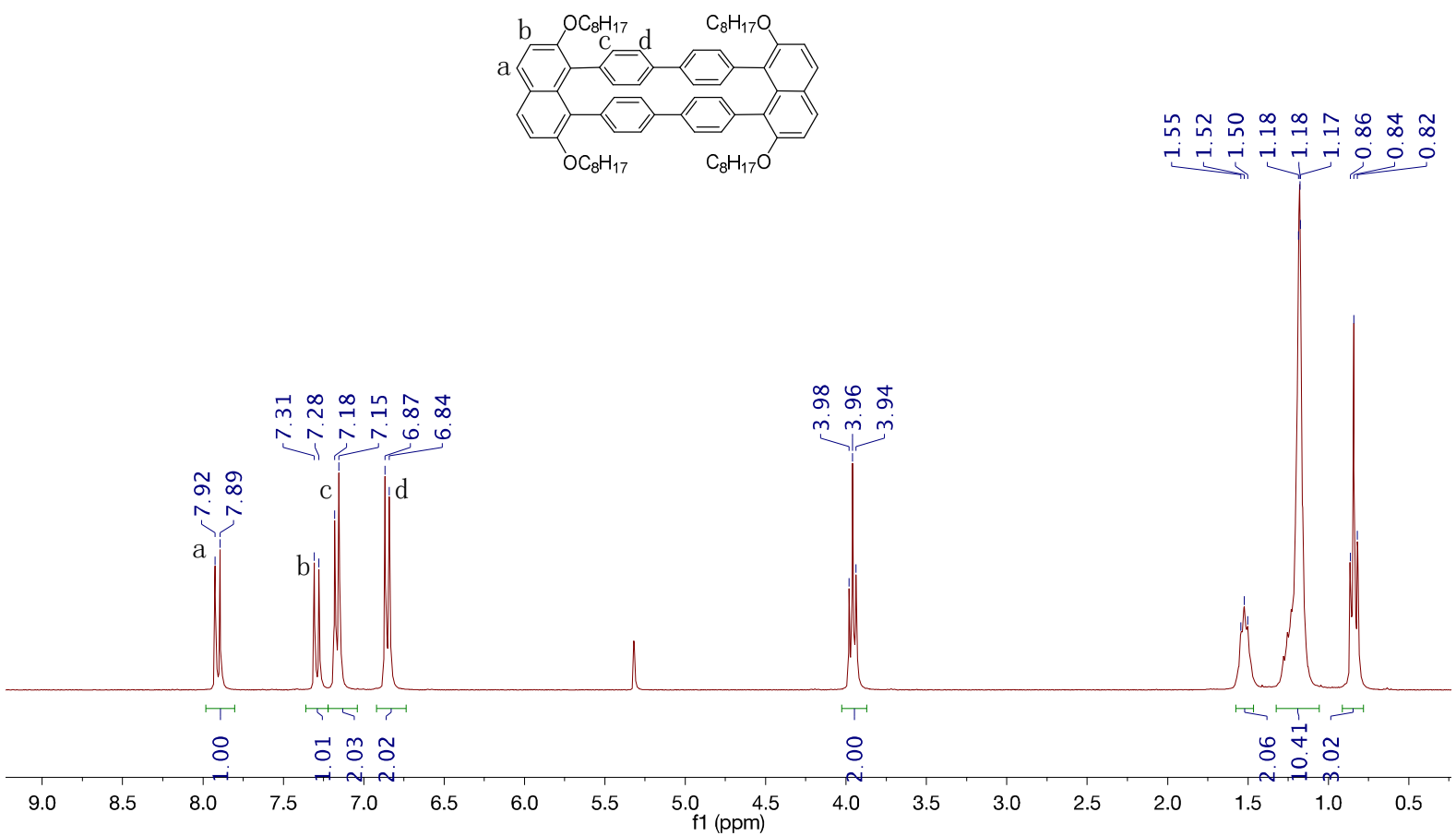

Figure S15. ${ }^{1} \mathrm{H}$ NMR spectrum of compound NP-1 (300 MHz, $\left.\mathrm{CD}_{2} \mathrm{Cl}_{2}, \mathrm{rt}\right)$

$1+1 \mathrm{M} 13 \mathrm{C} \mathrm{CD} 2 \mathrm{Cl} 2$

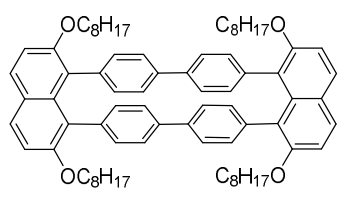

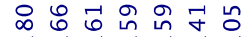

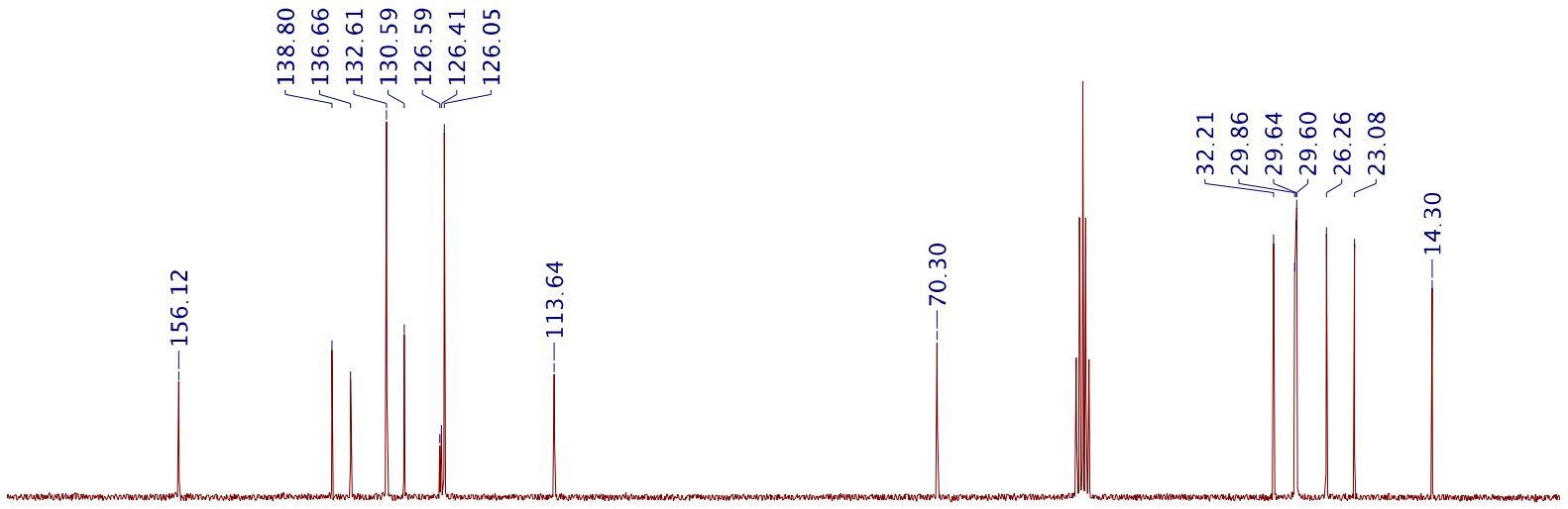

$\begin{array}{llllllllllllllllll}170 & 160 & 150 & 140 & 130 & 120 & 110 & 100 & \begin{array}{c}90 \\ \mathrm{f} 1(\mathrm{ppm})\end{array} & 80 & 70 & 60 & 50 & 40 & 30 & 20 & 10 & \mathrm{C}\end{array}$

Figure S16. ${ }^{13} \mathrm{C}$ NMR spectrum of compound NP-1 $\left(75 \mathrm{MHz}, \mathrm{CD}_{2} \mathrm{Cl}_{2}, \mathrm{rt}\right)$ 

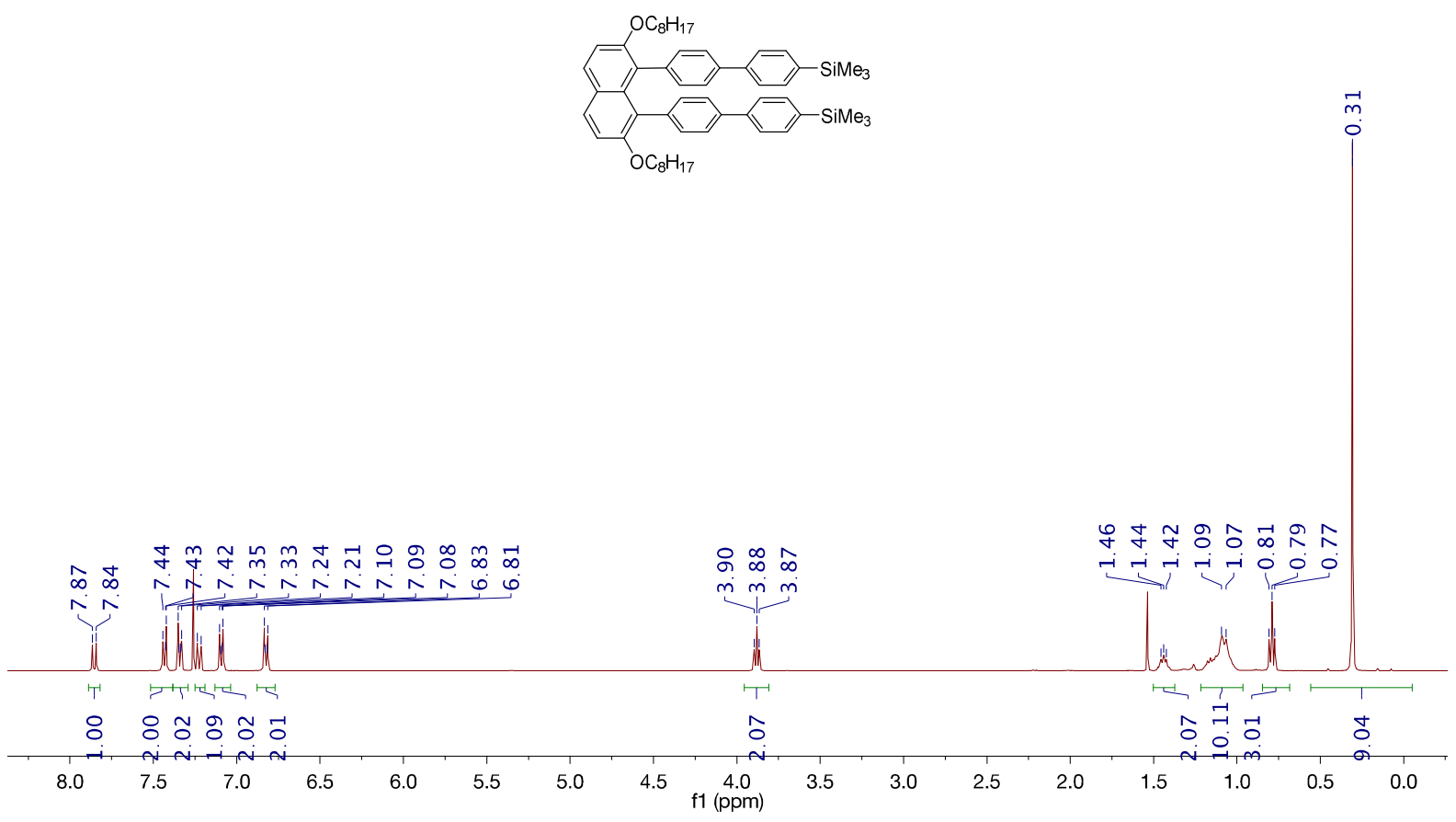

Figure S17. ${ }^{1} \mathrm{H}$ NMR spectrum of compound $\mathbf{3 b}\left(300 \mathrm{MHz}, \mathrm{CDCl}_{3}, \mathrm{rt}\right)$

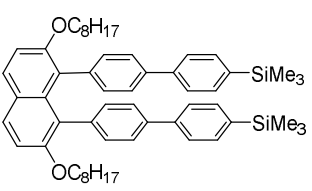

는 $\ln$ in

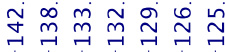
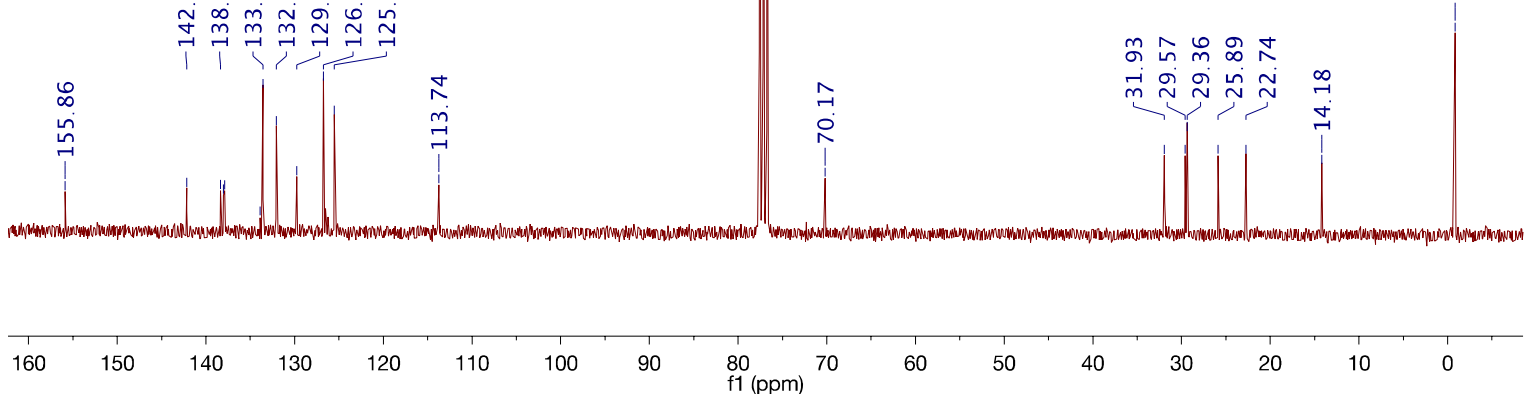

Figure S18. ${ }^{13} \mathrm{C}$ NMR spectrum of compound $\mathbf{3 b}\left(75 \mathrm{MHz}, \mathrm{CDCl}_{3}, \mathrm{rt}\right)$ 

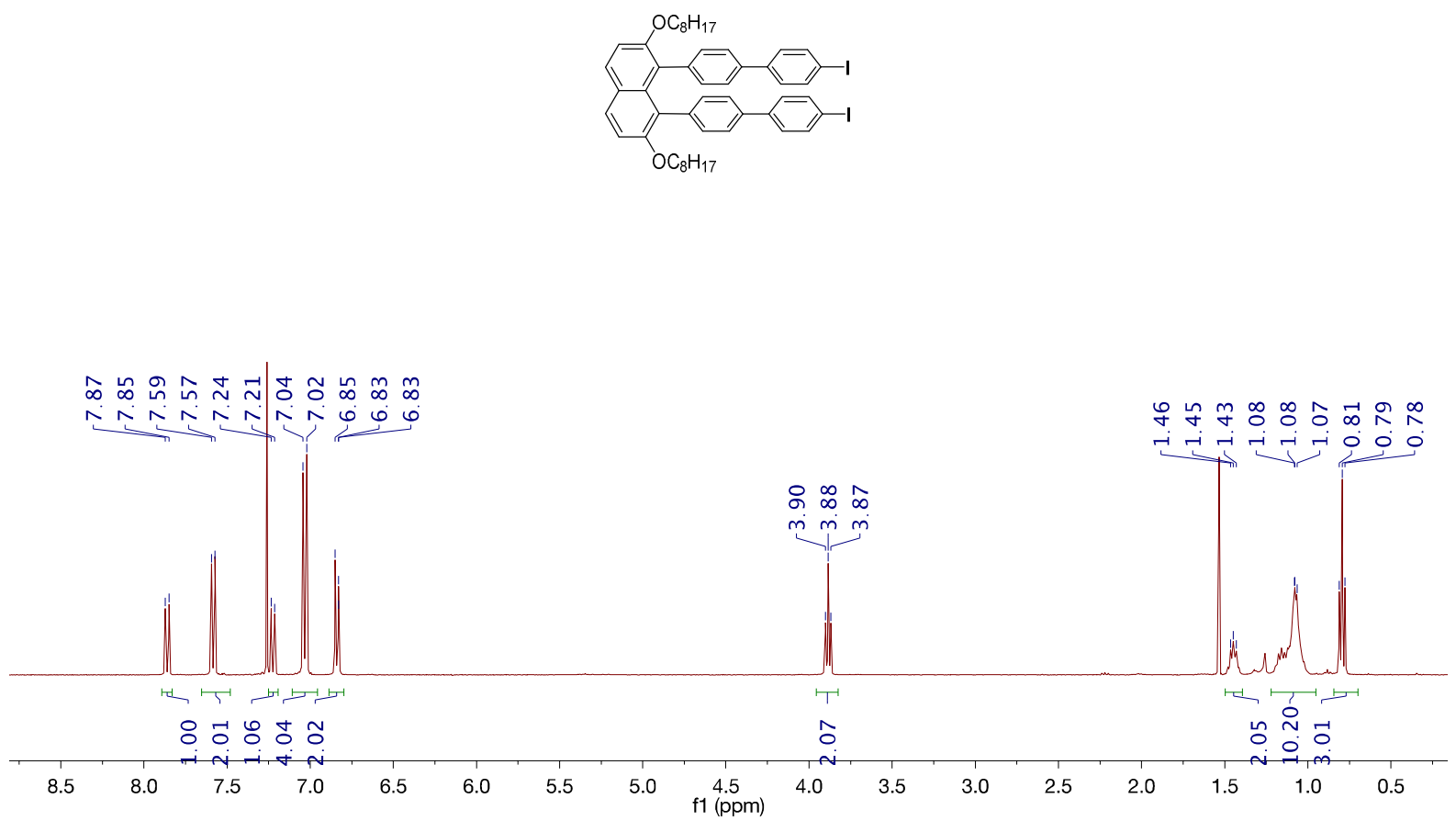

Figure S19. ${ }^{1} \mathrm{H}$ NMR spectrum of compound $4 \mathbf{b}\left(300 \mathrm{MHz}, \mathrm{CDCl}_{3}\right.$, rt)

biphenyl-I

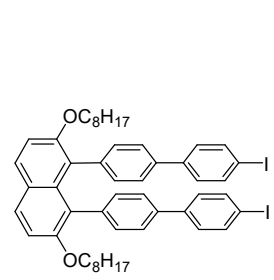

$\infty \stackrel{+}{\infty} \underset{0}{\infty} \stackrel{\infty}{\infty} \sim \sim 0$

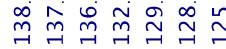

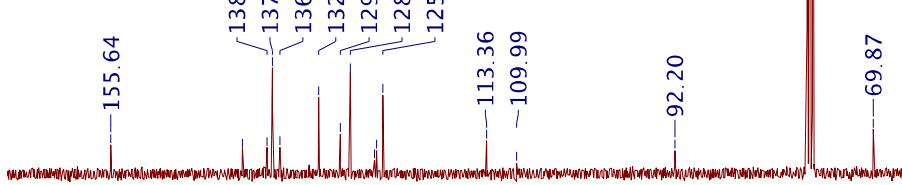

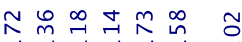

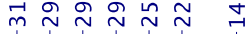

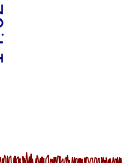

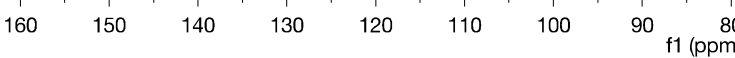

Figure S20. ${ }^{13} \mathrm{C}$ NMR spectrum of compound $4 \mathbf{b}\left(75 \mathrm{MHz}, \mathrm{CDCl}_{3}, \mathrm{rt}\right)$ 


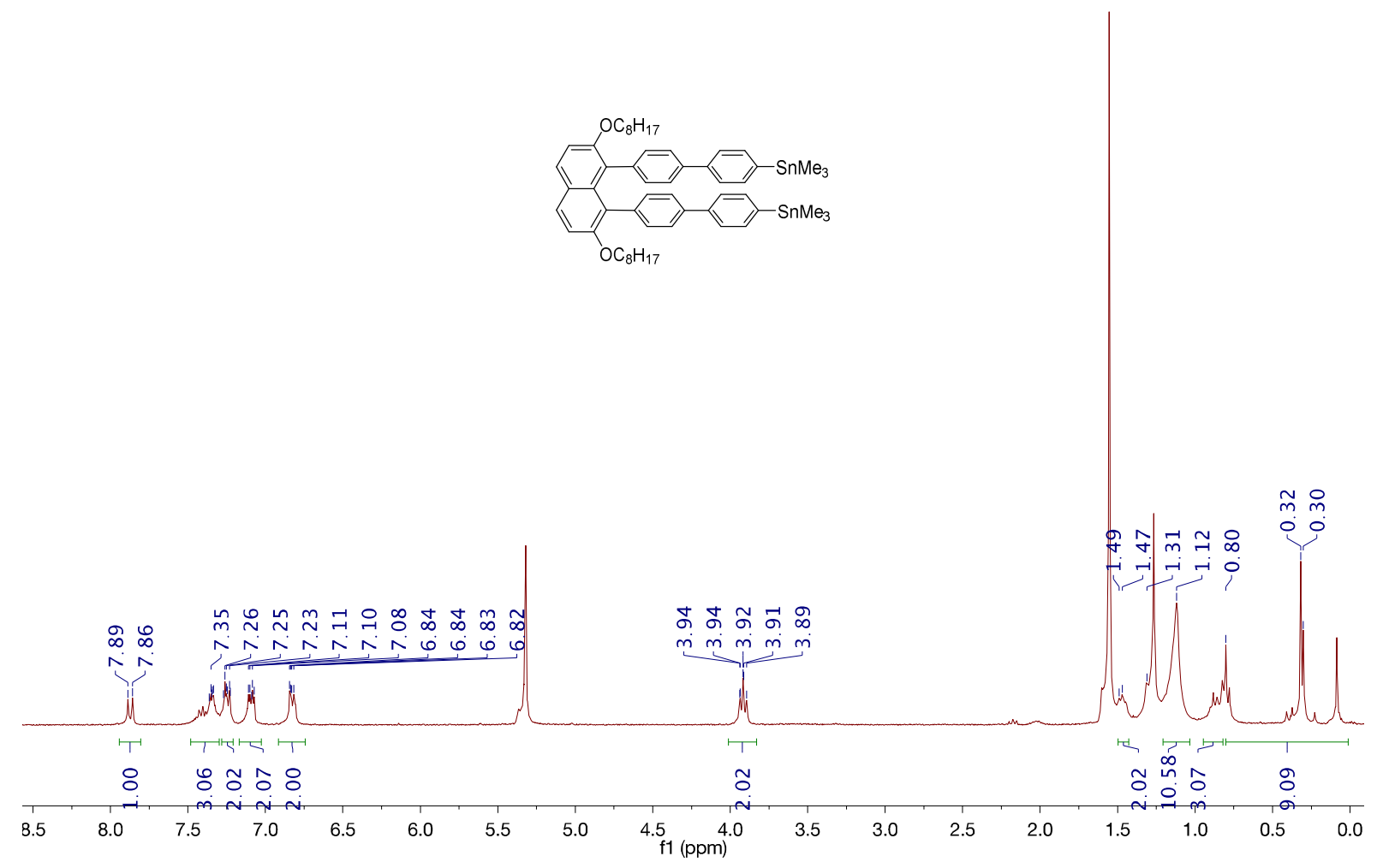

Figure S21. ${ }^{1} \mathrm{H}$ NMR spectrum of compound $\mathbf{5 b}\left(300 \mathrm{MHz}, \mathrm{CD}_{2} \mathrm{Cl}_{2}, \mathrm{rt}\right)$

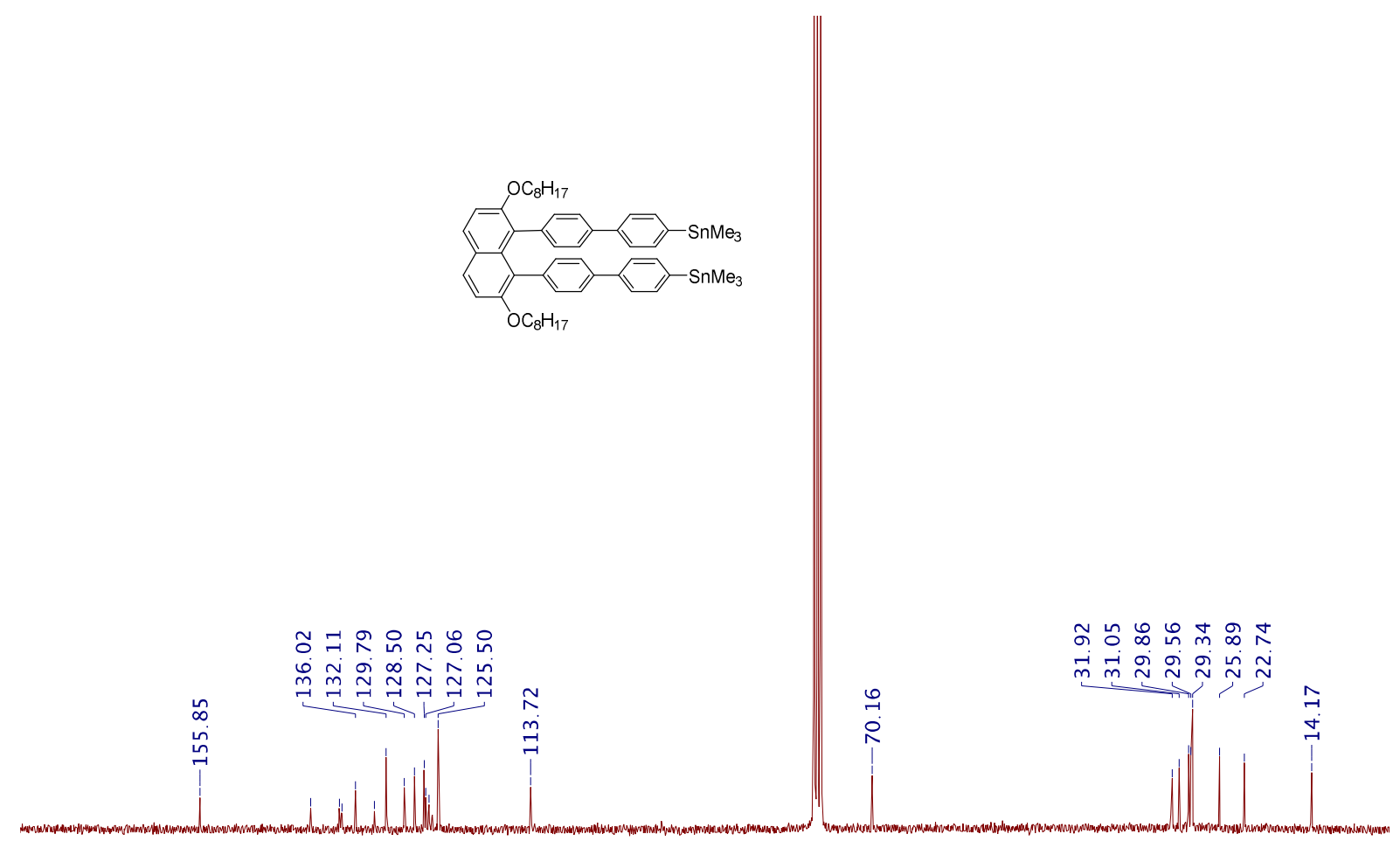

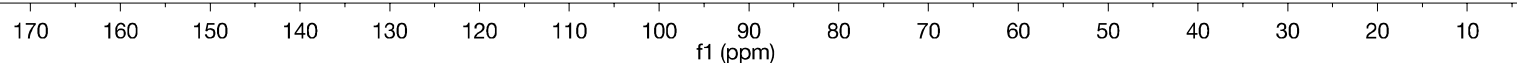

Figure S22. ${ }^{13} \mathrm{C}$ NMR spectrum of compound $\mathbf{5 b}\left(75 \mathrm{MHz}, \mathrm{CD}_{2} \mathrm{Cl}_{2}, \mathrm{rt}\right)$ 

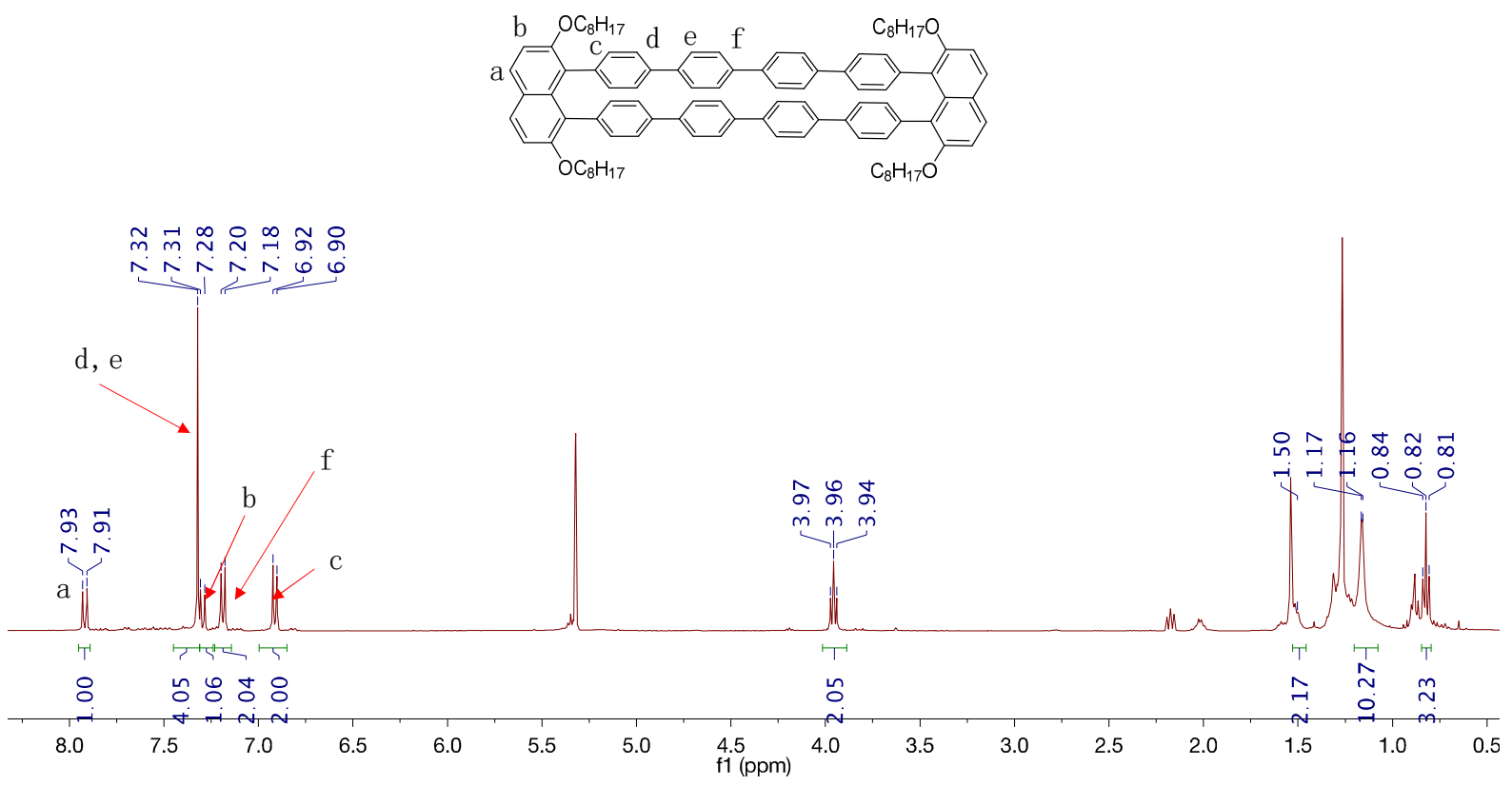

Figure S23. ${ }^{1} \mathrm{H}$ NMR spectrum of compound NP-2 (300 MHz, $\mathrm{CD}_{2} \mathrm{Cl}_{2}$, rt)

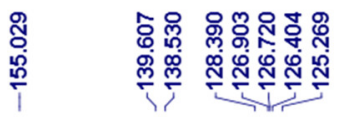

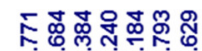

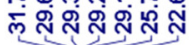
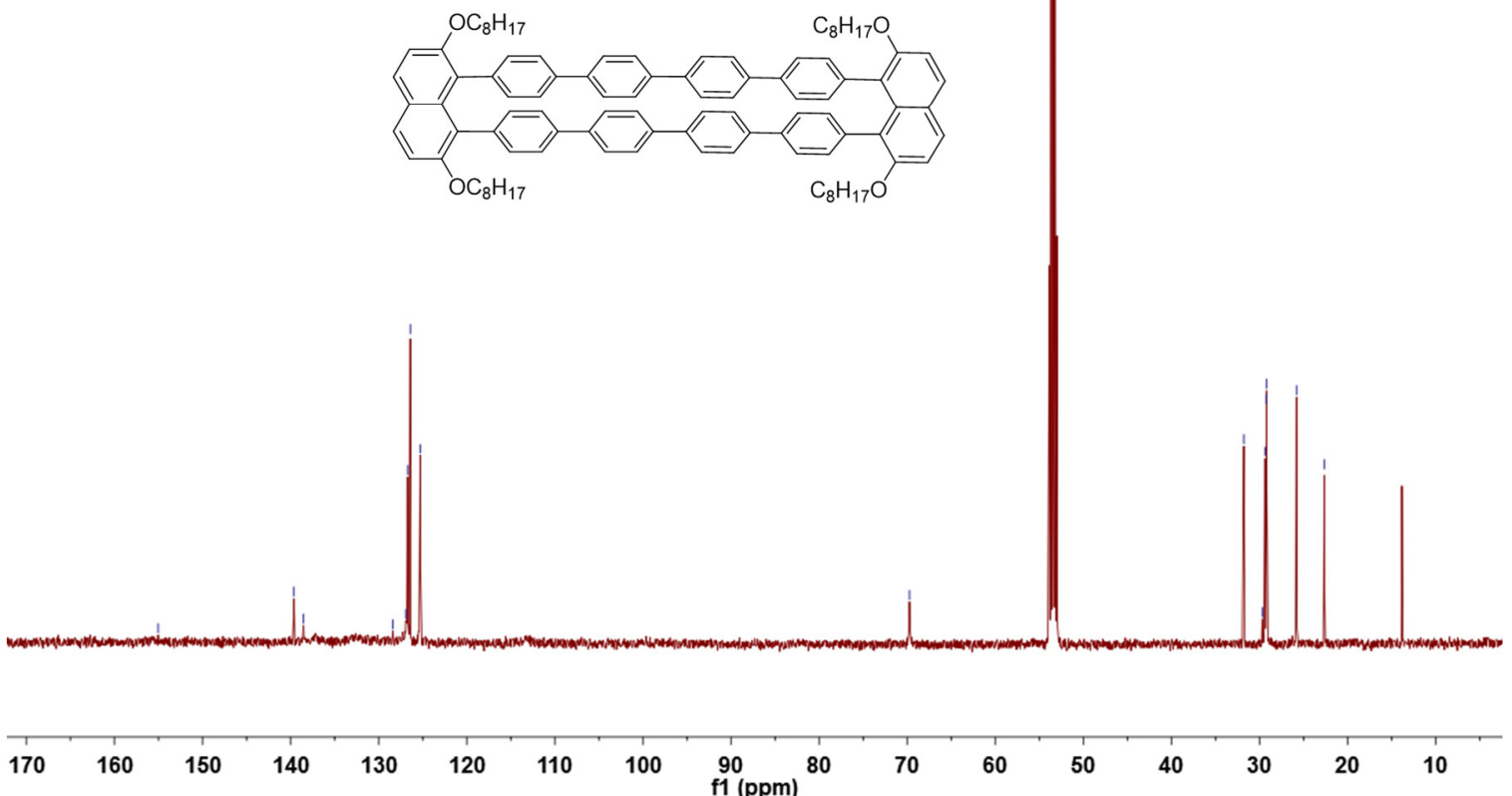

Figure S24. ${ }^{13} \mathrm{C}$ NMR spectrum of compound NP-2 $\left(75 \mathrm{MHz}, \mathrm{CD}_{2} \mathrm{Cl}_{2}\right.$, rt). The weak signal is due to the low solubility of the sample. 


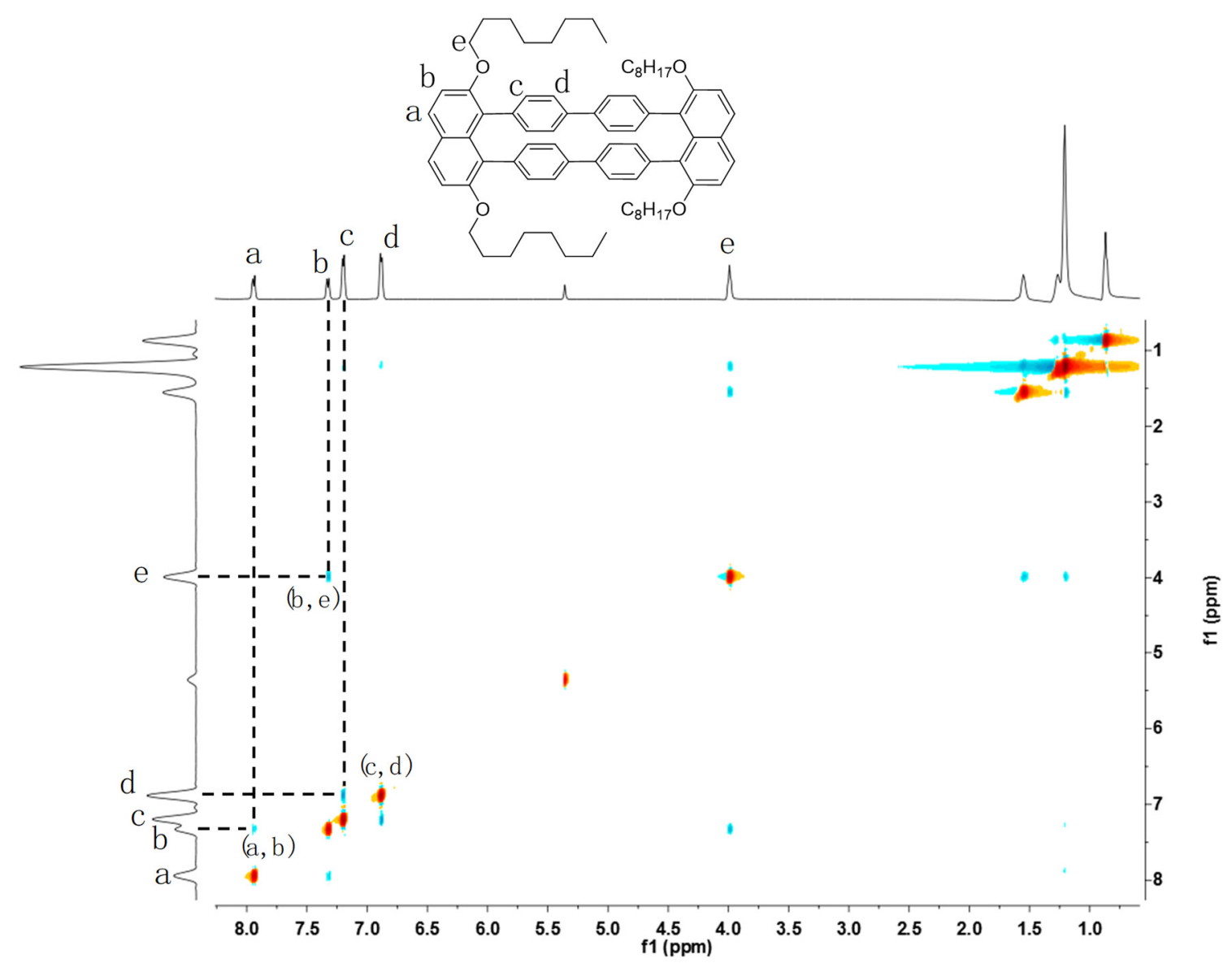

Figure S25. 2D NOSEY NMR spectrum of NP-1 (300 MHz, $\mathrm{CD}_{2} \mathrm{Cl}_{2}, \mathrm{rt}$ ).
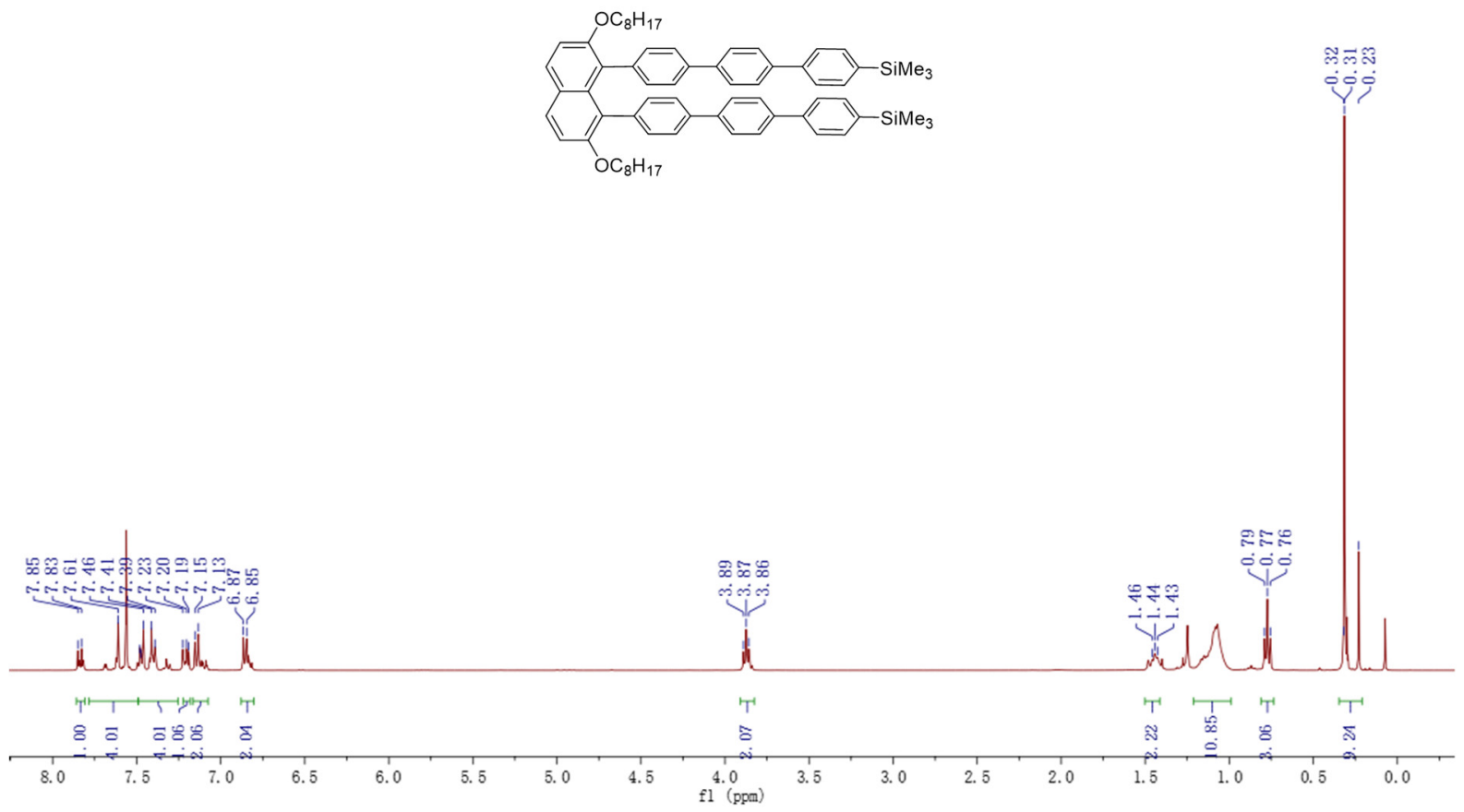

Figure S26. ${ }^{1} \mathrm{H}$ NMR spectrum of compound 3c (300 $\left.\mathrm{MHz}, \mathrm{CDCl}_{3}, \mathrm{rt}\right)$ 


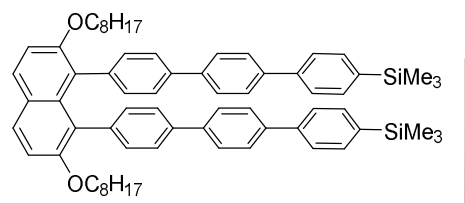

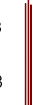$$
T
$$

160

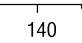

130

Figure S27. ${ }^{13} \mathrm{C}$ NMR spectrum of compound $\mathbf{3 c}\left(75 \mathrm{MHz}, \mathrm{CDCl}_{3}, \mathrm{rt}\right)$

triphenyl-I
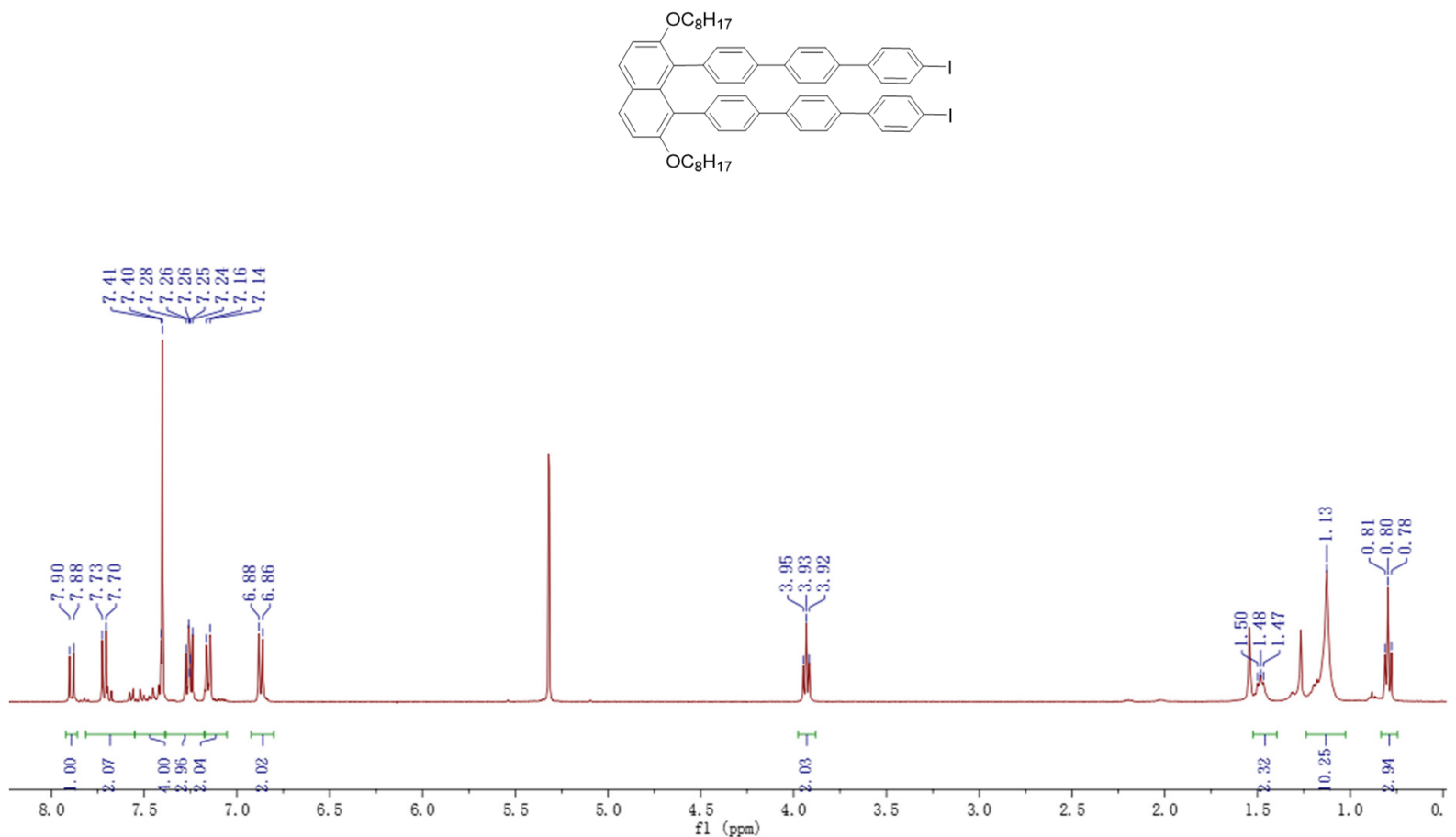

Figure S28. ${ }^{1} \mathrm{H}$ NMR spectrum of compound $4 \mathbf{c}\left(300 \mathrm{MHz}, \mathrm{CD}_{2} \mathrm{Cl}_{2}, \mathrm{rt}\right)$ 


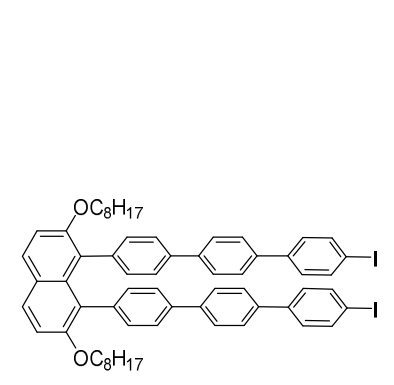

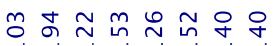

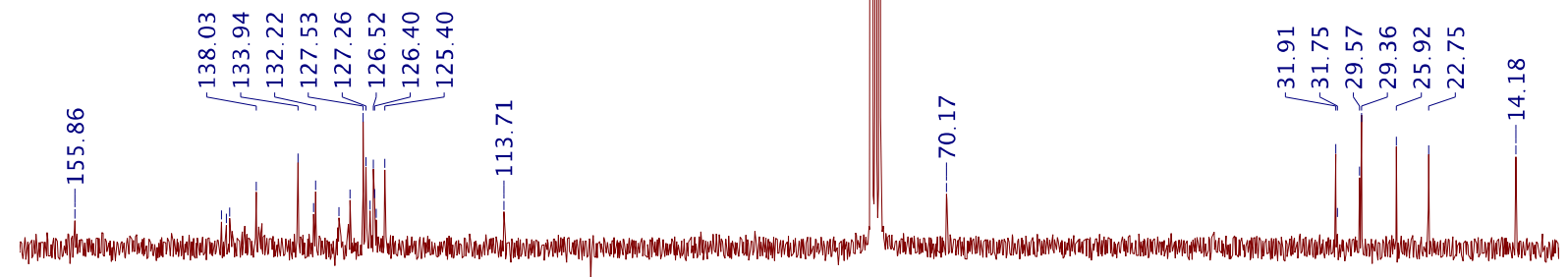

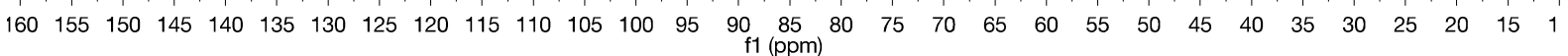

Figure S29. ${ }^{13} \mathrm{C}$ NMR spectrum of compound $4 \mathbf{c}\left(75 \mathrm{MHz}, \mathrm{CDCl}_{3}, \mathrm{rt}\right)$

Triphenyl-Snle 3-1H
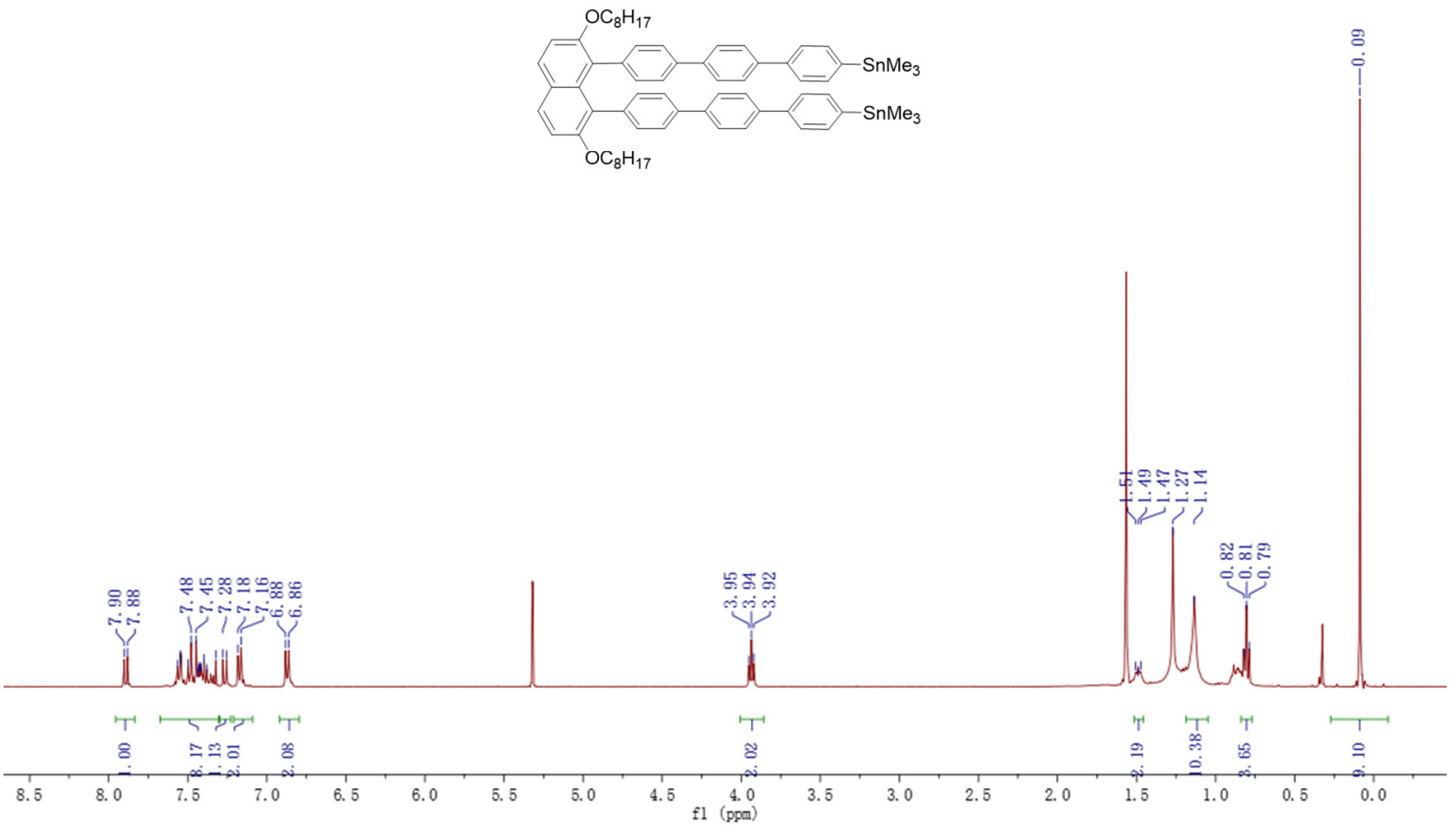

Figure S30. ${ }^{1} \mathrm{H}$ NMR spectrum of compound $\mathbf{5 c}\left(300 \mathrm{MHz}, \mathrm{CD}_{2} \mathrm{Cl}_{2}, \mathrm{rt}\right)$ 


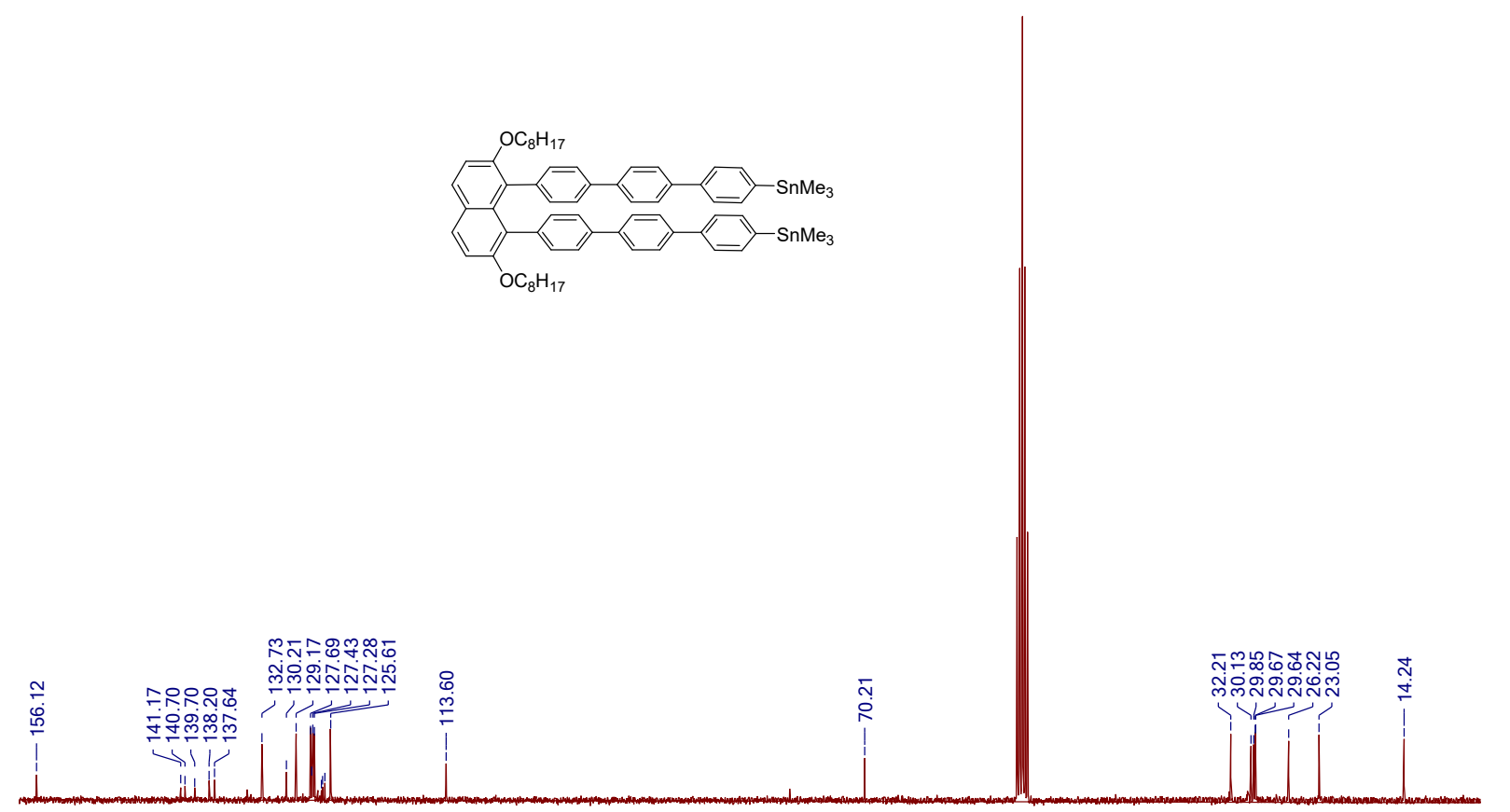

$\begin{array}{llllllllllllllllllllllllllllll}155 & 150 & 145 & 140 & 135 & 130 & 125 & 120 & 115 & 110 & 105 & 100 & 95 & 90 & \begin{array}{c}85 \\ \mathrm{f}(\mathrm{Dm})\end{array} & \mathbf{7 0} & 70 & 65 & 60 & 55 & 50 & 45 & 40 & 35 & 30 & 25 & 20 & 15 & 10\end{array}$

Figure S31. ${ }^{13} \mathrm{C}$ NMR spectrum of compound 5 c $\left(75 \mathrm{MHz}, \mathrm{CD}_{2} \mathrm{Cl}_{2}, \mathrm{rt}\right)$ 


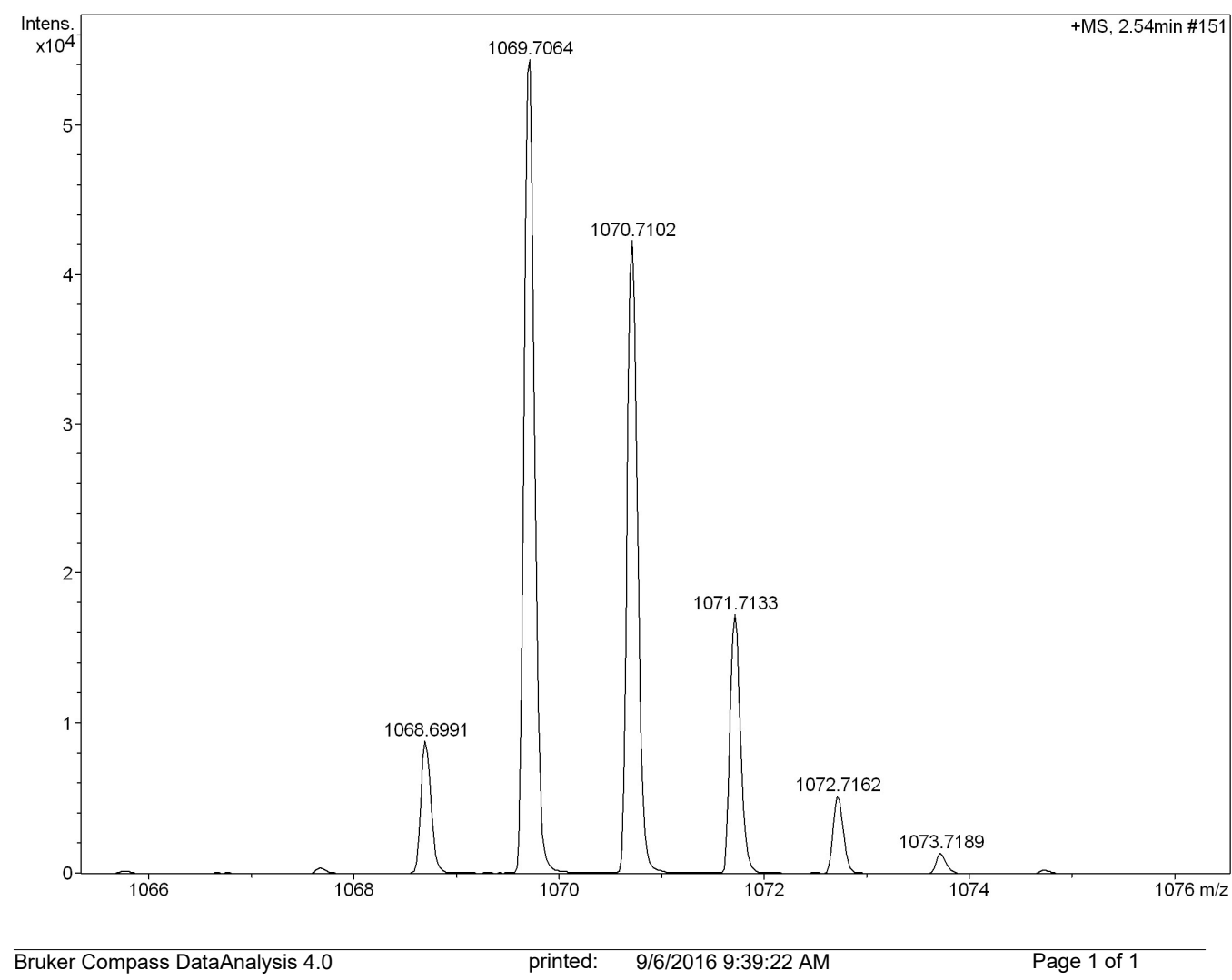

Figure S32. HR mass spectrum (APCI) of the compound NP-1. 


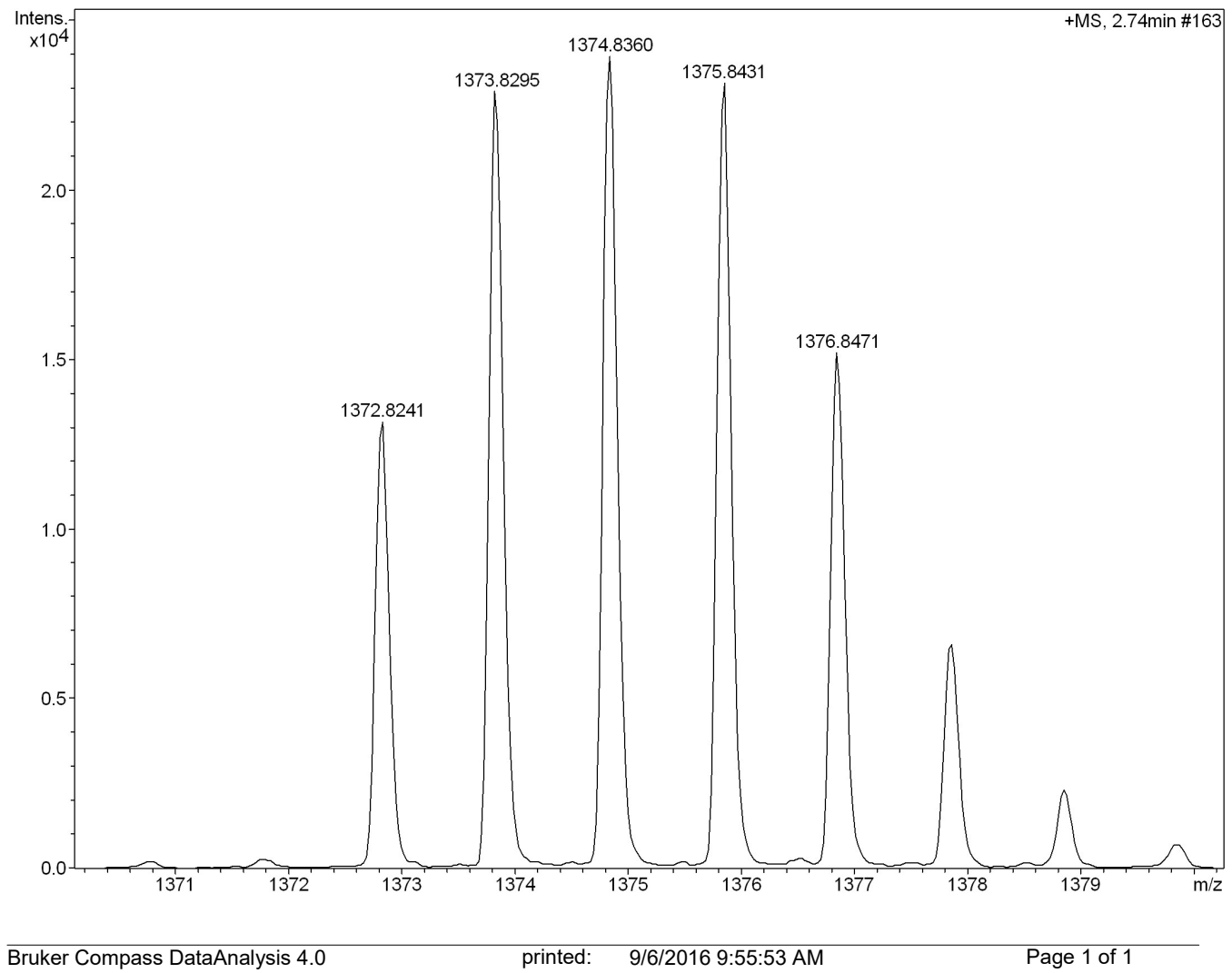

Figure S33. HR mass spectrum (APCI) of the compound NP-2.

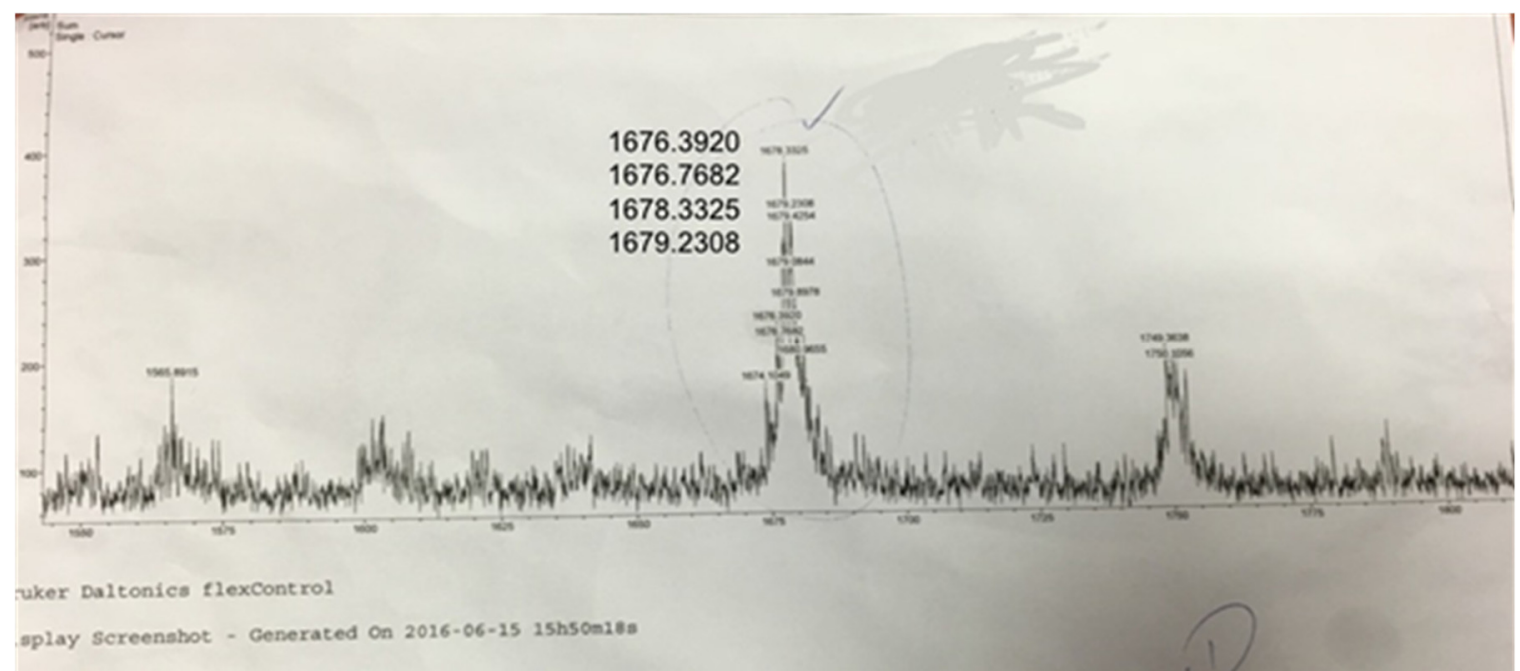

Figure S34. MALDI-TOF spectrum of the reaction mixture during the synthesis of compound NP-3. The major peak at m/z 1676 is correlated to the desirable NP-3. 


\section{References}

1. Thirsk, C; Hawkes, G. E.; Kroemer, R. T.; Liedl, K. R.; Loerting, T.; Nasser, R.; Pritchard,

R. G.; Steele, M.; Warren, J. E.; Whiting, A.; J. Chem. Soc., Perkin Trans. 2002, 2, 1510-1519.

2. Grüning, W. R.; Rossini, A. J.; Zagdoun, A.; Gajan, D.; Lesage, A.; Emsley, L.; Copéret, C.; Phys. Chem. Chem. Phys. 2013, 15, 13270-13274.

3. Gaussian 09; Revision A.2; Frisch, M. J.; Trucks, G. W.; Schlegel, H. B.; Scuseria, G. E.; Robb, M. A.; Cheeseman, J. R.; Scalmani, G.; Barone, V.; Mennucci, B.; Petersson, G. A.; Nakatsuji, H.; Caricato, M.; Li, X.; Hratchian, H. P.; Izmaylov, A. F.; Bloino, J.; Zheng, G.; Sonnenberg, J. L.; Hada, M.; Ehara, M.; Toyota, K.; Fukuda, R.; Hasegawa, J.; Ishida, M.; Nakajima, T.; Honda, Y.; Kitao, O.; Nakai, H.; Vreven, T.; Montgomery, J., J. A.; Peralta, J. E.; Ogliaro, F.; Bearpark, M.; Heyd, J. J.; Brothers, E.; Kudin, K. N.; Staroverov, V. N.; Kobayashi, R.; Normand, J.; Raghavachari, K.; Rendell, A.; Burant, J. C.; Iyengar, S. S.; Tomasi, J.; Cossi, M.; Rega, N.; Millam, N. J.; Klene, M.; Knox, J. E.; Cross, J. B.; Bakken, V.; Adamo, C.; Jaramillo, J.; Gomperts, R.; Stratmann, R. E.; Yazyev, O.; Austin, A. J.; Cammi, R.; Pomelli, C.; Ochterski, J. W.; Martin, R. L.; Morokuma, K.; Zakrzewski, V. G.; Voth, G. A.; Salvador, P.; Dannenberg, J. J.; Dapprich, S.; Daniels, A. D.; Farkas, Ö.; Foresman, J. B.; Ortiz, J. V.; Cioslowski, J.; Fox, D. J.; Gaussian, Inc., Wallingford CT, 2009.

4. (a) Becke, A. D. J. Chem. Phys. 1993, 98, 5648. (b) Lee, C.; Yang, W.; Parr, R. G. Phys. Rev. B: Condens. Matter 1988, 37, 785. (c) Yanai, T.; Tew, D.; and Handy, N. Chem. Phys. Lett. 2004, 393, 51. (d) Ditchfield, R.; Hehre, W. J.; Pople, J. A. J. Chem. Phys. 1971, 54, 724. (e) Hehre, W. J.; Ditchfield R.; Pople, J. A. J. Chem. Phys. 1972, 56, 2257. (f) Hariharan, P. C.; Pople, J. A. Theor. Chim. Acta 1973, 28, 213.

5. Fallah-Bagher-Shaidaei, H.; Wannere, S. S.; Corminboeuf, C.; Puchta, R.; Schleyer, P. V. R. Org. Lett. 2006, 8, 863.

6. Saint Program included in the package software: APEX3 v2016.1.0

7. SADABS. Ver. 2014/5. Krause, L., Herbs-Irmer, R., Sheldrick, G. M. Stalke, D. (2015). J. Appl. Crystallogr. 48.

8. SHELXT-Integrated space-group and crystal-structure determination Sheldrick, G. M. Acta Crystallogr., Sect. A 2015, A71, 3.

9. SHELXTL Sheldrick, G. M. Ver. 2014/7. Acta Crystallographica. Sect C Structural Chemistry 71, 3 .

10. WinGX Farrugia, L. J. J. Appl. Cryst. 1999, 32, 837. 


\section{Cartesian coordinates and energies of optimised geometries}

Cartesian coordinates of NP-1 (at RB3LYP/6-31G(d), $\mathrm{OC}_{8} \mathrm{H}_{17}$ is replaced by $\mathrm{OCH}_{3}$ )

\section{$\mathrm{O}$}

$\mathrm{O}$

$\mathrm{O}$

$\mathrm{O}$

$\mathrm{C}$

$\mathrm{C}$

$\mathrm{C}$

$\mathrm{H}$

$\mathrm{C}$

$\mathrm{H}$

C

$\mathrm{C}$

$\mathrm{H}$

C

$\mathrm{H}$

C

$\mathrm{C}$

C

C

C

$\mathrm{H}$

C

$\mathrm{H}$

C

C

$\mathrm{H}$

C

$\mathrm{H}$

C

C

$\mathrm{H}$

$\mathrm{C}$

$\mathrm{H}$

C

C

$\mathrm{H}$

C

$\mathrm{H}$

C

C
$-5.11555062$

$-5.11552824$

5.11552053

5.11569163

$-5.03673355$

$-5.81056765$

$-7.22434902$

$-7.79912172$

$-7.86109642$

$-8.94762161$

$-7.13895606$

$-7.86108610$

$-8.94761172$

$-7.22432066$

$-7.79907526$

$-5.81054103$

$-5.03671411$

$-5.69138259$

$-3.55456506$

$-2.90641086$

$-3.49069134$

$-1.53045349$

$-1.05761263$

$-0.74200417$

$-1.40815233$

$-0.83181823$

$-2.78606336$

$-3.26580517$

0.74193983

1.53030413

1.05738294

2.90625410

3.49045499

3.55452218

2.78611772

3.26591862

1.40819061

0.83193883

$-3.55455059$

$-2.78608704$
$-3.63005673$

3.63014608

$-3.63012473$

3.63018128

$-1.28773000$

$-2.45311974$

$-2.42623703$

$-3.34346347$

$-1.21474492$

$-1.17075260$

0.00002736

1.21480192

1.17081195

2.42629985

3.34353601

2.45318810

1.28779763

0.00003062

$-1.51427537$

$-2.01132446$

$-2.22222803$

$-2.21695817$

$-2.58840761$

$-1.93409594$

$-1.51989029$

$-1.28607499$

$-1.32122738$

$-0.96803741$

$-1.93412933$

$-2.21782119$

$-2.58987526$

$-2.01222699$

$-2.22373730$

$-1.51436377$

$-1.32054187$

$-0.96671093$

$-1.51918574$

$-1.28473041$

1.51428858

1.32111073
$-0.16427036$

0.16376232

0.16430795

$-0.16373670$

$-0.04920697$

$-0.09914101$

$-0.09530736$

$-0.13524604$

$-0.04561881$

$-0.04288255$

0.00031300

0.04639833

0.04406908

0.09569482

0.13567110

0.09906776

0.04913451

0.00008590

$-0.05402334$

1.08678195

1.97867257

1.10234884

2.00781617

$-0.02637605$

$-1.19140285$

$-2.08160386$

$-1.20664800$

$-2.11502242$

0.02744573

$-1.10113796$

$-2.00631351$

$-1.08581227$

$-1.97760619$

0.05458942

1.20714684

2.11523781

1.19214313

2.08223074

0.05383559

1.20648196 


\begin{tabular}{|c|c|c|c|}
\hline $\mathrm{H}$ & -3.26588851 & 0.96785245 & 2.11479701 \\
\hline $\mathrm{C}$ & -1.40817524 & 1.51973486 & 1.19131452 \\
\hline $\mathrm{H}$ & -0.83187720 & 1.28583673 & 2.08151716 \\
\hline $\mathrm{C}$ & -0.74197374 & 1.93401887 & 0.02633981 \\
\hline $\mathrm{C}$ & -1.53036913 & 2.21696858 & -1.10239393 \\
\hline $\mathrm{H}$ & -1.05747937 & 2.58844951 & -2.00782304 \\
\hline $\mathrm{C}$ & -2.90634046 & 2.01139009 & -1.08689868 \\
\hline $\mathrm{H}$ & -3.49056427 & 2.22237779 & -1.97880249 \\
\hline $\mathrm{C}$ & 0.74197625 & 1.93407097 & -0.02741638 \\
\hline $\mathrm{C}$ & 1.40829778 & 1.51906327 & -1.19205305 \\
\hline $\mathrm{H}$ & 0.83209663 & 1.28451737 & -2.08215021 \\
\hline $\mathrm{C}$ & 2.78622644 & 1.32044636 & -1.20695467 \\
\hline $\mathrm{H}$ & 3.26610214 & 0.96656274 & -2.11498550 \\
\hline $\mathrm{C}$ & 3.55457359 & 1.51439959 & -0.05436834 \\
\hline $\mathrm{C}$ & 2.90623503 & 2.01233693 & 1.08594920 \\
\hline $\mathrm{H}$ & 3.49037252 & 2.22395715 & 1.97775327 \\
\hline $\mathrm{C}$ & 1.53027330 & 2.21787341 & 1.10118388 \\
\hline $\mathrm{H}$ & 1.05728615 & 2.58997546 & 2.00630498 \\
\hline $\mathrm{C}$ & 5.03666565 & -1.28778811 & 0.04934765 \\
\hline $\mathrm{C}$ & 5.81052251 & -2.45317595 & 0.09904982 \\
\hline $\mathrm{C}$ & 7.22430362 & -2.42630349 & 0.09504613 \\
\hline $\mathrm{H}$ & 7.79906690 & -3.34353901 & 0.13484215 \\
\hline $\mathrm{C}$ & 7.86104943 & -1.21481311 & 0.04552288 \\
\hline $\mathrm{H}$ & 8.94757267 & -1.17080984 & 0.04276568 \\
\hline $\mathrm{C}$ & 7.13890473 & -0.00003312 & -0.00020529 \\
\hline $\mathrm{C}$ & 7.86109113 & 1.21471058 & -0.04605558 \\
\hline $\mathrm{H}$ & 8.94761362 & 1.17067334 & -0.04363813 \\
\hline $\mathrm{C}$ & 7.22438062 & 2.42624009 & -0.09523502 \\
\hline $\mathrm{H}$ & 7.79917453 & 3.34345993 & -0.13501005 \\
\hline $\mathrm{C}$ & 5.81061422 & 2.45317841 & -0.09886950 \\
\hline $\mathrm{C}$ & 5.03670592 & 1.28781135 & -0.04916989 \\
\hline $\mathrm{C}$ & 5.69132809 & 0.00000192 & -0.00000650 \\
\hline $\mathrm{C}$ & -5.82743941 & -4.85327759 & -0.22498656 \\
\hline $\mathrm{H}$ & -6.46109863 & -4.91408028 & -1.11993991 \\
\hline $\mathrm{H}$ & -6.44827482 & -5.01112466 & 0.66715688 \\
\hline $\mathrm{H}$ & -5.06558194 & -5.63377329 & -0.27277881 \\
\hline $\mathrm{H}$ & -5.06558879 & 5.63390601 & 0.27145463 \\
\hline $\mathrm{H}$ & 5.06589006 & 5.63393331 & -0.27158729 \\
\hline $\mathrm{H}$ & 5.06548294 & -5.63389034 & 0.27178408 \\
\hline $\mathrm{C}$ & -5.82743576 & 4.85337241 & 0.22411963 \\
\hline $\mathrm{H}$ & -6.46091009 & 4.91454310 & 1.11917444 \\
\hline $\mathrm{H}$ & -6.44846236 & 5.01081802 & -0.66796396 \\
\hline
\end{tabular}




$\begin{array}{lrrr}\mathrm{C} & 5.82736734 & -4.85340548 & 0.22423767 \\ \mathrm{H} & 6.46121193 & -4.91469615 & 1.11903123 \\ \mathrm{H} & 6.44800636 & -5.01083707 & -0.66811189 \\ \mathrm{C} & 5.82768437 & 4.85336134 & -0.22404136 \\ \mathrm{H} & 6.46134486 & 4.91441372 & -1.11897187 \\ \mathrm{H} & 6.44853607 & 5.01084824 & 0.66815547\end{array}$

Cartesian coordinates of $\mathbf{N P - 1} \mathbf{1}^{2+}$ (singlet, optimized at UCAM/6-31G(d), $\mathrm{OC}_{8} \mathrm{H}_{17}$ is replaced by $\mathrm{OCH}_{3}$ )

\begin{tabular}{|c|c|c|c|}
\hline $\mathrm{O}$ & 5.11018802 & -3.50580715 & -0.86544122 \\
\hline $\mathrm{O}$ & 5.11022185 & 3.50580306 & 0.86543909 \\
\hline $\mathrm{O}$ & -5.11021829 & -3.50587851 & 0.86513586 \\
\hline $\mathrm{O}$ & -5.11019209 & 3.50588689 & -0.86512604 \\
\hline $\mathrm{C}$ & 4.98430029 & -1.24918993 & -0.27453510 \\
\hline $\mathrm{C}$ & 5.78922747 & -2.39267324 & -0.62321513 \\
\hline $\mathrm{C}$ & 7.18109328 & -2.33786929 & -0.65808962 \\
\hline $\mathrm{H}$ & 7.76852003 & -3.21125416 & -0.90542131 \\
\hline $\mathrm{C}$ & 7.80234118 & -1.15469442 & -0.33237762 \\
\hline $\mathrm{H}$ & 8.88689191 & -1.10537237 & -0.32922466 \\
\hline $\mathrm{C}$ & 7.07855302 & -0.00000869 & -0.00001228 \\
\hline $\mathrm{C}$ & 7.80235081 & 1.15467245 & 0.33235217 \\
\hline $\mathrm{H}$ & 8.88690117 & 1.10534276 & 0.32919223 \\
\hline $\mathrm{C}$ & 7.18111376 & 2.33785006 & 0.65807334 \\
\hline $\mathrm{H}$ & 7.76854787 & 3.21123065 & 0.90540295 \\
\hline $\mathrm{C}$ & 5.78924816 & 2.39266277 & 0.62321047 \\
\hline $\mathrm{C}$ & 4.98430945 & 1.24918549 & 0.27452830 \\
\hline $\mathrm{C}$ & 5.63881476 & -0.00000397 & -0.00000888 \\
\hline $\mathrm{C}$ & 3.52981957 & -1.51090681 & -0.16952516 \\
\hline $\mathrm{C}$ & 2.81213937 & -1.98929423 & -1.27259902 \\
\hline $\mathrm{H}$ & 3.32611356 & -2.18854121 & -2.20610644 \\
\hline $\mathrm{C}$ & 1.44245623 & -2.17090456 & -1.20115962 \\
\hline $\mathrm{H}$ & 0.91096675 & -2.52453186 & -2.07832574 \\
\hline $\mathrm{C}$ & 0.74088886 & -1.89983380 & -0.02110712 \\
\hline $\mathrm{C}$ & 1.47788805 & -1.51299749 & 1.10345784 \\
\hline $\mathrm{H}$ & 0.96797963 & -1.30068259 & 2.03660300 \\
\hline $\mathrm{C}$ & 2.84785781 & -1.31880110 & 1.03234481 \\
\hline $\mathrm{H}$ & 3.38757442 & -0.98782168 & 1.91331495 \\
\hline $\mathrm{C}$ & -0.74089913 & -1.89983943 & 0.02094174 \\
\hline $\mathrm{C}$ & -1.44246306 & -2.17097847 & 1.20098070 \\
\hline $\mathrm{H}$ & -0.91096951 & -2.52464908 & 2.07812690 \\
\hline $\mathrm{C}$ & -2.81214664 & -1.98937900 & 1.27243283 \\
\hline $\mathrm{H}$ & -3.32611678 & -2.18867565 & 2.20593157 \\
\hline $\mathrm{C}$ & -3.52983077 & -1.51092910 & 0.16938774 \\
\hline
\end{tabular}




\begin{tabular}{|c|c|c|c|}
\hline $\mathrm{C}$ & -2.84787158 & -1.31875411 & -1.03247347 \\
\hline $\mathrm{H}$ & -3.38759113 & -0.98772603 & -1.91342321 \\
\hline $\mathrm{C}$ & -1.47790237 & -1.51294591 & -1.10360133 \\
\hline $\mathrm{H}$ & -0.96799665 & -1.30057944 & -2.03673612 \\
\hline $\mathrm{C}$ & 3.52983079 & 1.51090889 & 0.16952550 \\
\hline $\mathrm{C}$ & 2.84786802 & 1.31883030 & -1.03234890 \\
\hline $\mathrm{H}$ & 3.38758491 & 0.98787466 & -1.91332761 \\
\hline $\mathrm{C}$ & 1.47789856 & 1.51302751 & -1.10345715 \\
\hline $\mathrm{H}$ & 0.96799028 & 1.30073689 & -2.03660787 \\
\hline $\mathrm{C}$ & 0.74089866 & 1.89983302 & 0.02111806 \\
\hline $\mathrm{C}$ & 1.44246522 & 2.17087370 & 1.20117811 \\
\hline $\mathrm{H}$ & 0.91097413 & 2.52447427 & 2.07835409 \\
\hline $\mathrm{C}$ & 2.81214882 & 1.98926566 & 1.27261264 \\
\hline $\mathrm{H}$ & 3.32612121 & 2.18848579 & 2.20612652 \\
\hline $\mathrm{C}$ & -0.74088911 & 1.89983715 & -0.02093145 \\
\hline $\mathrm{C}$ & -1.47789207 & 1.51292478 & 1.10360497 \\
\hline $\mathrm{H}$ & -0.96798642 & 1.30054414 & 2.03673673 \\
\hline $\mathrm{C}$ & -2.84786214 & 1.31873686 & 1.03247433 \\
\hline $\mathrm{H}$ & -3.38758211 & 0.98769625 & 1.91341920 \\
\hline $\mathrm{C}$ & -3.52982004 & 1.51092799 & -0.16938449 \\
\hline $\mathrm{C}$ & -2.81213588 & 1.98939327 & -1.27242205 \\
\hline $\mathrm{H}$ & -3.32610648 & 2.18870588 & -2.20591727 \\
\hline $\mathrm{C}$ & -1.44245255 & 2.17099476 & -1.20096611 \\
\hline $\mathrm{H}$ & -0.91095902 & 2.52468064 & -2.07810623 \\
\hline $\mathrm{C}$ & -4.98430783 & -1.24921069 & 0.27441863 \\
\hline $\mathrm{C}$ & -5.78924567 & -2.39271751 & 0.62300430 \\
\hline $\mathrm{C}$ & -7.18111122 & -2.33790746 & 0.65787489 \\
\hline $\mathrm{H}$ & -7.76854512 & -3.21130942 & 0.90512922 \\
\hline $\mathrm{C}$ & -7.80234859 & -1.15470172 & 0.33225750 \\
\hline $\mathrm{H}$ & -8.88689895 & -1.10537181 & 0.32910330 \\
\hline $\mathrm{C}$ & -7.07855132 & 0.00000858 & -0.00000662 \\
\hline $\mathrm{C}$ & -7.80234081 & 1.15472283 & -0.33226960 \\
\hline $\mathrm{H}$ & -8.88689143 & 1.10539959 & -0.32911968 \\
\hline $\mathrm{C}$ & -7.18109434 & 2.33792662 & -0.65787815 \\
\hline $\mathrm{H}$ & -7.76852231 & 3.21133287 & -0.90513139 \\
\hline $\mathrm{C}$ & -5.78922858 & 2.39272916 & -0.62299999 \\
\hline $\mathrm{C}$ & -4.98429986 & 1.24921653 & -0.27441953 \\
\hline $\mathrm{C}$ & -5.63881291 & 0.00000469 & -0.00000456 \\
\hline $\mathrm{C}$ & 5.79949912 & -4.71770410 & -1.18211743 \\
\hline $\mathrm{H}$ & 6.44965184 & -5.01667793 & -0.35549111 \\
\hline $\mathrm{H}$ & 6.37951014 & -4.60467081 & -2.10204453 \\
\hline $\mathrm{H}$ & 5.01959066 & -5.46253487 & -1.32575820 \\
\hline
\end{tabular}




$\begin{array}{lrrc}\mathrm{H} & 5.01964858 & 5.46253233 & 1.32575573 \\ \mathrm{H} & -5.01960097 & 5.46265599 & -1.32526872 \\ \mathrm{H} & -5.01964289 & -5.46264753 & 1.32528311 \\ \mathrm{C} & 5.79954806 & 4.71769305 & 1.18211061 \\ \mathrm{H} & 6.44969899 & 5.01665882 & 0.35548000 \\ \mathrm{H} & 6.37956348 & 4.60465327 & 2.10203410 \\ \mathrm{C} & -5.79954314 & -4.71779655 & 1.18170308 \\ \mathrm{H} & -6.44969433 & -5.01669157 & 0.35504709 \\ \mathrm{H} & -6.37955808 & -4.60483681 & 2.10163665 \\ \mathrm{C} & -5.79950705 & 4.71781033 & -1.18169259 \\ \mathrm{H} & -6.44965856 & 5.01670891 & -0.35503813 \\ \mathrm{H} & -6.37952003 & 4.60485746 & -2.10162825\end{array}$

Cartesian coordinates of $\mathbf{N P - 1} \mathbf{1}^{2+}$ (triplet, optimized at UCAM/6-31G(d), $\mathrm{OC}_{8} \mathrm{H}_{17}$ is replaced by $\mathrm{OCH}_{3}$ )

$\begin{array}{lccc}\mathrm{O} & 5.11050367 & -3.52096609 & -0.79630056 \\ \mathrm{O} & 5.11073233 & 3.52091036 & 0.79634206 \\ \mathrm{O} & -5.11072627 & -3.52096972 & 0.79606052 \\ \mathrm{O} & -5.11049616 & 3.52102351 & -0.79600870 \\ \mathrm{C} & 4.98672324 & -1.25327602 & -0.25250796 \\ \mathrm{C} & 5.79122733 & -2.40480165 & -0.57425129 \\ \mathrm{C} & 7.18317141 & -2.35195231 & -0.60729866 \\ \mathrm{H} & 7.77048788 & -3.23069144 & -0.83524176 \\ \mathrm{C} & 7.80467141 & -1.16197437 & -0.30704551 \\ \mathrm{H} & 8.88924574 & -1.11300505 & -0.30454335 \\ \mathrm{C} & 7.08121224 & -0.00005675 & -0.00014935 \\ \mathrm{C} & 7.80473999 & 1.16180982 & 0.30677812 \\ \mathrm{H} & 8.88931152 & 1.11277936 & 0.30426074 \\ \mathrm{C} & 7.18331170 & 2.35179722 & 0.60714250 \\ \mathrm{H} & 7.77068209 & 3.23048929 & 0.83512826 \\ \mathrm{C} & 5.79136915 & 2.40471550 & 0.57418329 \\ \mathrm{C} & 4.98678918 & 1.25325962 & 0.25234849 \\ \mathrm{C} & 5.64142890 & -0.00001426 & -0.00014776 \\ \mathrm{C} & 3.53012432 & -1.51104988 & -0.15376598 \\ \mathrm{C} & 2.81851748 & -1.99320840 & -1.25855628 \\ \mathrm{H} & 3.33766481 & -2.19706760 & -2.18836897 \\ \mathrm{C} & 1.44827892 & -2.17491253 & -1.19298886 \\ \mathrm{H} & 0.92076555 & -2.53234905 & -2.07106009 \\ \mathrm{C} & 0.74132607 & -1.89885767 & -0.01758735 \\ \mathrm{C} & 1.47228770 & -1.50717401 & 1.10867194 \\ \mathrm{H} & 0.95734435 & -1.29046193 & 2.03807701 \\ \mathrm{C} & 2.84310194 & -1.31431768 & 1.04390023 \\ \mathrm{H} & 3.37857590 & -0.97972951 & 1.92610157\end{array}$




\begin{tabular}{|c|c|c|c|}
\hline $\mathrm{C}$ & -0.74138011 & -1.89885979 & 0.01766334 \\
\hline $\mathrm{C}$ & -1.44834060 & -2.17445231 & 1.19316922 \\
\hline $\mathrm{H}$ & -0.92082959 & -2.53151988 & 2.07139166 \\
\hline $\mathrm{C}$ & -2.81858719 & -1.99277124 & 1.25864016 \\
\hline $\mathrm{H}$ & -3.33773794 & -2.19626623 & 2.18852886 \\
\hline $\mathrm{C}$ & -3.53019294 & -1.51106520 & 0.15364736 \\
\hline $\mathrm{C}$ & -2.84315017 & -1.31477043 & -1.04408000 \\
\hline $\mathrm{H}$ & -3.37861859 & -0.98055326 & -1.92642375 \\
\hline $\mathrm{C}$ & -1.47233674 & -1.50764696 & -1.10876207 \\
\hline $\mathrm{H}$ & -0.95738728 & -1.29130976 & -2.03825082 \\
\hline $\mathrm{C}$ & 3.53019178 & 1.51107832 & 0.15374682 \\
\hline $\mathrm{C}$ & 2.84316093 & 1.31495081 & -1.04401553 \\
\hline $\mathrm{H}$ & 3.37863772 & 0.98085592 & -1.92640032 \\
\hline $\mathrm{C}$ & 1.47234630 & 1.50782461 & -1.10868192 \\
\hline $\mathrm{H}$ & 0.95740551 & 1.29160495 & -2.03820269 \\
\hline $\mathrm{C}$ & 0.74137748 & 1.89887299 & 0.01779329 \\
\hline $\mathrm{C}$ & 1.44832772 & 2.17431523 & 1.19334009 \\
\hline $\mathrm{H}$ & 0.92080652 & 2.53125344 & 2.07160906 \\
\hline $\mathrm{C}$ & 2.81857493 & 1.99263716 & 1.25879642 \\
\hline $\mathrm{H}$ & 3.33771497 & 2.19600182 & 2.18871937 \\
\hline $\mathrm{C}$ & -0.74132880 & 1.89884592 & -0.01745516 \\
\hline $\mathrm{C}$ & -1.47227492 & 1.50699525 & 1.10875554 \\
\hline $\mathrm{H}$ & -0.95732024 & 1.29016364 & 2.03812656 \\
\hline $\mathrm{C}$ & -2.84308802 & 1.31413519 & 1.04397156 \\
\hline $\mathrm{H}$ & -3.37855091 & 0.97942229 & 1.92613252 \\
\hline $\mathrm{C}$ & -3.53012558 & 1.51103638 & -0.15365736 \\
\hline $\mathrm{C}$ & -2.81853354 & 1.99334571 & -1.25839143 \\
\hline $\mathrm{H}$ & -3.33769459 & 2.19733750 & -2.18816767 \\
\hline $\mathrm{C}$ & -1.44829553 & 2.17505337 & -1.19281296 \\
\hline $\mathrm{H}$ & -0.92079526 & 2.53262187 & -2.07083826 \\
\hline $\mathrm{C}$ & -4.98679434 & -1.25327345 & 0.25225994 \\
\hline $\mathrm{C}$ & -5.79136984 & -2.40476186 & 0.57398762 \\
\hline $\mathrm{C}$ & -7.18331339 & -2.35185117 & 0.60694524 \\
\hline $\mathrm{H}$ & -7.77068167 & -3.23056457 & 0.83485417 \\
\hline $\mathrm{C}$ & -7.80474544 & -1.16183860 & 0.30668487 \\
\hline $\mathrm{H}$ & -8.88931708 & -1.11281087 & 0.30417111 \\
\hline $\mathrm{C}$ & -7.08122044 & 0.00005672 & -0.00014184 \\
\hline $\mathrm{C}$ & -7.80467692 & 1.16200330 & -0.30693634 \\
\hline $\mathrm{H}$ & -8.88925141 & 1.11303701 & -0.30443662 \\
\hline $\mathrm{C}$ & -7.18317316 & 2.35200614 & -0.60708662 \\
\hline $\mathrm{H}$ & -7.77048746 & 3.23076654 & -0.83495291 \\
\hline $\mathrm{C}$ & -5.79122797 & 2.40484739 & -0.57404245 \\
\hline
\end{tabular}




$\begin{array}{lrrc}\mathrm{C} & -4.98672862 & 1.25328921 & -0.25240698 \\ \mathrm{C} & -5.64143826 & 0.00001400 & -0.00014126 \\ \mathrm{C} & 5.79849945 & -4.74002184 & -1.08752174 \\ \mathrm{H} & 6.44603121 & -5.02397904 & -0.25357453 \\ \mathrm{H} & 6.38108185 & -4.64562737 & -2.00793344 \\ \mathrm{H} & 5.01766831 & -5.48615146 & -1.21878689 \\ \mathrm{H} & 5.01805860 & 5.48607320 & 1.21897282 \\ \mathrm{H} & -5.01764436 & 5.48624105 & -1.21834117 \\ \mathrm{H} & -5.01803948 & -5.48616565 & 1.21853411 \\ \mathrm{C} & 5.79882934 & 4.73989574 & 1.08762028 \\ \mathrm{H} & 6.44634512 & 5.02386484 & 0.25366480 \\ \mathrm{H} & 6.38144576 & 4.64539273 & 2.00799928 \\ \mathrm{C} & -5.79881552 & -4.73998440 & 1.08723469 \\ \mathrm{H} & -6.44632130 & -5.02389203 & 0.25325058 \\ \mathrm{H} & -6.38144089 & -4.64556029 & 2.00761607 \\ \mathrm{C} & -5.79848214 & 4.74010944 & -1.08712688 \\ \mathrm{H} & -6.44600226 & 5.02400787 & -0.25315065 \\ \mathrm{H} & -6.38107486 & 4.64579366 & -2.00754006\end{array}$

Cartesian coordinates of NP-2 (optimized at RB3LYP/6-31G(d), $\mathrm{OC}_{8} \mathrm{H}_{17}$ is replaced by $\mathrm{OCH}_{3}$ )

$\begin{array}{lrrr}\text { O } & -9.30419532 & 3.39413095 & -0.01812356 \\ \mathrm{O} & -9.18802747 & -3.86319586 & 0.02370735 \\ \mathrm{C} & -9.19433717 & 1.04905189 & -0.01215247 \\ \mathrm{C} & -9.98102159 & 2.20705327 & 0.01280162 \\ \mathrm{C} & -11.39488356 & 2.15890542 & 0.04530798 \\ \mathrm{H} & -11.98119719 & 3.06925970 & 0.06477065 \\ \mathrm{C} & -12.01692353 & 0.93857215 & 0.03098484 \\ \mathrm{H} & -13.10282435 & 0.88134522 & 0.03855602 \\ \mathrm{C} & -11.27943153 & -0.26752167 & 0.00630330 \\ \mathrm{C} & -11.97919944 & -1.49587607 & -0.01760898 \\ \mathrm{H} & -13.06634456 & -1.47211083 & -0.02446992 \\ \mathrm{C} & -11.31981141 & -2.69667120 & -0.03252526 \\ \mathrm{H} & -11.87764625 & -3.62496025 & -0.05288491 \\ \mathrm{C} & -9.90571475 & -2.69891054 & -0.00034563 \\ \mathrm{C} & -9.15567119 & -1.51776989 & 0.02793963 \\ \mathrm{C} & -9.83300606 & -0.24445359 & 0.00651466 \\ \mathrm{C} & -7.71825539 & 1.28931046 & -0.11929121 \\ \mathrm{C} & -7.05915634 & 1.17191098 & -1.34909739 \\ \mathrm{H} & -7.61852977 & 0.85100950 & -2.22380829 \\ \mathrm{C} & -5.69954388 & 1.44863455 & -1.46457716 \\ \mathrm{H} & -5.20928539 & 1.33950577 & -2.42841982 \\ \mathrm{C} & -4.95100186 & 1.87635757 & -0.35727809 \\ \mathrm{C} & -5.62470076 & 2.04659299 & 0.86329214\end{array}$




\begin{tabular}{|c|c|c|c|}
\hline $\mathrm{H}$ & -5.06750853 & 2.36871539 & 1.73940325 \\
\hline $\mathrm{C}$ & -6.98110021 & 1.75594232 & 0.97820402 \\
\hline $\mathrm{H}$ & -7.47768734 & 1.87891766 & 1.93696989 \\
\hline $\mathrm{C}$ & -3.48352893 & 2.07110237 & -0.45764811 \\
\hline $\mathrm{C}$ & -2.82810333 & 3.17236880 & 0.11700860 \\
\hline $\mathrm{H}$ & -3.41528482 & 3.95216873 & 0.59549639 \\
\hline $\mathrm{C}$ & -1.44160418 & 3.29521143 & 0.06198511 \\
\hline $\mathrm{H}$ & -0.97374842 & 4.17718072 & 0.49088336 \\
\hline $\mathrm{C}$ & -0.64392247 & 2.31256958 & -0.55193105 \\
\hline $\mathrm{C}$ & -1.30675673 & 1.21901247 & -1.13730149 \\
\hline $\mathrm{H}$ & -0.72928008 & 0.41222465 & -1.57721618 \\
\hline $\mathrm{C}$ & -2.69123612 & 1.10645563 & -1.10050929 \\
\hline $\mathrm{H}$ & -3.16503361 & 0.22359775 & -1.51908221 \\
\hline $\mathrm{C}$ & 0.83849568 & 2.37346203 & -0.54172692 \\
\hline $\mathrm{C}$ & 1.60147349 & 1.74109937 & -1.53909544 \\
\hline $\mathrm{H}$ & 1.10161144 & 1.27199531 & -2.38108589 \\
\hline $\mathrm{C}$ & 2.98749718 & 1.68030319 & -1.46504888 \\
\hline $\mathrm{H}$ & 3.52611509 & 1.12334044 & -2.22516551 \\
\hline $\mathrm{C}$ & 3.69481334 & 2.27047955 & -0.40270404 \\
\hline $\mathrm{C}$ & 2.93622547 & 2.96300431 & 0.55827089 \\
\hline $\mathrm{H}$ & 3.43856319 & 3.47037039 & 1.37679412 \\
\hline $\mathrm{C}$ & 1.54646553 & 3.00347219 & 0.49701809 \\
\hline $\mathrm{H}$ & 1.00098365 & 3.50297679 & 1.29264729 \\
\hline $\mathrm{C}$ & 5.16316400 & 2.09664063 & -0.27187730 \\
\hline $\mathrm{C}$ & 5.97983172 & 1.84904332 & -1.38863209 \\
\hline $\mathrm{H}$ & 5.55269841 & 1.87976848 & -2.38672919 \\
\hline $\mathrm{C}$ & 7.33751173 & 1.57437990 & -1.24680542 \\
\hline $\mathrm{H}$ & 7.94034352 & 1.38365165 & -2.13068898 \\
\hline $\mathrm{C}$ & 7.94259278 & 1.54289399 & 0.01569674 \\
\hline $\mathrm{C}$ & 7.14464059 & 1.84555010 & 1.12701138 \\
\hline $\mathrm{H}$ & 7.59324886 & 1.85344697 & 2.11659909 \\
\hline $\mathrm{C}$ & 5.78579041 & 2.10463877 & 0.98957626 \\
\hline $\mathrm{H}$ & 5.19194955 & 2.27614698 & 1.88272630 \\
\hline $\mathrm{C}$ & -10.02575591 & 4.62355714 & -0.04105707 \\
\hline $\mathrm{H}$ & -10.64417657 & 4.71609630 & 0.86345685 \\
\hline $\mathrm{H}$ & -10.69574151 & 4.65181646 & -0.91232174 \\
\hline $\mathrm{C}$ & -9.00259699 & 5.74444500 & -0.11131366 \\
\hline $\mathrm{H}$ & -8.39362468 & 5.65243266 & -1.01570967 \\
\hline $\mathrm{H}$ & -9.50871069 & 6.71588753 & -0.12665745 \\
\hline $\mathrm{H}$ & -8.33572608 & 5.71107153 & 0.75567822 \\
\hline $\mathrm{H}$ & 9.36304671 & 5.63772593 & 0.75528593 \\
\hline $\mathrm{C}$ & -9.87601097 & -5.10193918 & 0.02881822 \\
\hline
\end{tabular}




\begin{tabular}{|c|c|c|c|}
\hline $\mathrm{H}$ & -10.47847218 & -5.23973713 & -0.87903680 \\
\hline $\mathrm{H}$ & -10.52286597 & -5.20667675 & 0.91002945 \\
\hline $\mathrm{H}$ & -9.09940362 & -5.86847192 & 0.06193582 \\
\hline $\mathrm{H}$ & 9.57085837 & -5.53697312 & -0.74033732 \\
\hline $\mathrm{O}$ & 9.58255150 & -3.54335545 & -0.50583489 \\
\hline $\mathrm{O}$ & 9.45138798 & 3.64761016 & 0.50715502 \\
\hline $\mathrm{C}$ & 9.46299591 & -1.22279679 & -0.15557800 \\
\hline $\mathrm{C}$ & 10.25350538 & -2.35920803 & -0.36891914 \\
\hline $\mathrm{C}$ & 11.66621692 & -2.30100203 & -0.40837905 \\
\hline $\mathrm{H}$ & 12.25466678 & -3.19552892 & -0.57138183 \\
\hline $\mathrm{C}$ & 12.28649129 & -1.09681677 & -0.20448306 \\
\hline $\mathrm{H}$ & 13.37222431 & -1.03682143 & -0.20483175 \\
\hline $\mathrm{C}$ & 11.54637198 & 0.08859684 & 0.00869696 \\
\hline $\mathrm{C}$ & 12.24345786 & 1.29917101 & 0.22620121 \\
\hline $\mathrm{H}$ & 13.33058705 & 1.27639667 & 0.23457795 \\
\hline $\mathrm{C}$ & 11.58096641 & 2.48173381 & 0.42402082 \\
\hline $\mathrm{H}$ & 12.13671889 & 3.39624974 & 0.59099995 \\
\hline $\mathrm{C}$ & 10.16749397 & 2.48987027 & 0.37327873 \\
\hline $\mathrm{C}$ & 9.41946235 & 1.32659284 & 0.15427150 \\
\hline $\mathrm{C}$ & 10.10022761 & 0.06302208 & 0.00300509 \\
\hline $\mathrm{C}$ & 7.99304735 & -1.47934089 & -0.02239592 \\
\hline $\mathrm{C}$ & 7.36469307 & -1.40667851 & 1.22716368 \\
\hline $\mathrm{H}$ & 7.94588188 & -1.11733047 & 2.09856945 \\
\hline $\mathrm{C}$ & 6.00814598 & -1.68781887 & 1.36700530 \\
\hline $\mathrm{H}$ & 5.54215779 & -1.61207653 & 2.34596492 \\
\hline $\mathrm{C}$ & 5.23184437 & -2.07566400 & 0.26445256 \\
\hline $\mathrm{C}$ & 5.87461688 & -2.20212724 & -0.97805935 \\
\hline $\mathrm{H}$ & 5.29508593 & -2.49282604 & -1.85066928 \\
\hline $\mathrm{C}$ & 7.22705973 & -1.90581917 & -1.11720448 \\
\hline $\mathrm{H}$ & 7.69735543 & -1.99178502 & -2.09277690 \\
\hline $\mathrm{C}$ & 3.76626451 & -2.26932301 & 0.39228472 \\
\hline $\mathrm{C}$ & 3.09472494 & -3.35610068 & -0.19102594 \\
\hline $\mathrm{H}$ & 3.66885414 & -4.12845617 & -0.69665821 \\
\hline $\mathrm{C}$ & 1.70899686 & -3.47400958 & -0.11087902 \\
\hline $\mathrm{H}$ & 1.22940140 & -4.34592363 & -0.54706655 \\
\hline $\mathrm{C}$ & 0.92743608 & -2.49929662 & 0.53589459 \\
\hline $\mathrm{C}$ & 1.60720774 & -1.42060500 & 1.12966012 \\
\hline $\mathrm{H}$ & 1.04328512 & -0.61885229 & 1.59543871 \\
\hline $\mathrm{C}$ & 2.99136725 & -1.31450039 & 1.06979624 \\
\hline $\mathrm{H}$ & 3.47810789 & -0.44321335 & 1.49762522 \\
\hline $\mathrm{C}$ & -0.55540179 & -2.55324239 & 0.55167093 \\
\hline $\mathrm{C}$ & -1.29807287 & -1.91731298 & 1.56207073 \\
\hline
\end{tabular}




$\begin{array}{lrrr}\mathrm{H} & -0.78208821 & -1.45004677 & 2.39517488 \\ \mathrm{C} & -2.68481777 & -1.85093145 & 1.51362964 \\ \mathrm{H} & -3.20524567 & -1.29120993 & 2.28413711 \\ \mathrm{C} & -3.41533258 & -2.44088694 & 0.46687950 \\ \mathrm{C} & -2.67644980 & -3.13715006 & -0.50689006 \\ \mathrm{H} & -3.19454692 & -3.64592030 & -1.31450118 \\ \mathrm{C} & -1.28587431 & -3.18117312 & -0.47275544 \\ \mathrm{H} & -0.75851639 & -3.68360226 & -1.27862449 \\ \mathrm{C} & -4.88655261 & -2.26749122 & 0.36671487 \\ \mathrm{C} & -5.67486755 & -1.98560901 & 1.49608265 \\ \mathrm{H} & -5.22331288 & -1.98640061 & 2.48372475 \\ \mathrm{C} & -7.03540761 & -1.71429749 & 1.38100918 \\ \mathrm{H} & -7.61529872 & -1.49720345 & 2.27420540 \\ \mathrm{C} & -7.67334904 & -1.72153489 & 0.13441729 \\ \mathrm{C} & -6.90503162 & -2.05964242 & -0.98673076 \\ \mathrm{H} & -7.37956158 & -2.10007636 & -1.96351904 \\ \mathrm{C} & -5.54224849 & -2.31385112 & -0.87684351 \\ \mathrm{H} & -4.97336052 & -2.51363633 & -1.78014329 \\ \mathrm{C} & 10.31750339 & -4.74351596 & -0.67122185 \\ \mathrm{H} & 10.91742407 & -4.73364447 & -1.59111717 \\ \mathrm{H} & 10.97478070 & -4.94174320 & 0.18579914 \\ \mathrm{C} & 10.13939281 & 4.87314849 & 0.68814945 \\ \mathrm{H} & 10.73029260 & 4.87874781 & 1.61388527 \\ \mathrm{H} & 10.79717172 & 5.10205479 & -0.16084346\end{array}$

Cartesian coordinates of $\mathbf{N P - 2} \mathbf{2}^{2+}$ (singlet, optimized at UCAM/6-31G(d), $\mathrm{OC}_{8} \mathrm{H}_{17}$ is replaced by $\mathrm{OCH}_{3}$ )

$\begin{array}{llrc}\mathrm{O} & -9.26871346 & 3.32783486 & 0.51413397 \\ \mathrm{O} & -9.07921625 & -3.81029490 & -0.53143679 \\ \mathrm{C} & -9.08521590 & 1.03058343 & 0.08483032 \\ \mathrm{C} & -9.91116612 & 2.17787508 & 0.37457131 \\ \mathrm{C} & -11.30354550 & 2.08759243 & 0.43262722 \\ \mathrm{H} & -11.90862090 & 2.96292422 & 0.62222984 \\ \mathrm{C} & -11.90193845 & 0.87519335 & 0.18830005 \\ \mathrm{H} & -12.98545453 & 0.80487005 & 0.18933560 \\ \mathrm{C} & -11.15524945 & -0.28946330 & -0.05523320 \\ \mathrm{C} & -11.84358163 & -1.48588671 & -0.29605639 \\ \mathrm{H} & -12.92887400 & -1.46755185 & -0.31693903 \\ \mathrm{C} & -11.18070748 & -2.67316663 & -0.50440692 \\ \mathrm{H} & -11.73830758 & -3.58096915 & -0.68930875 \\ \mathrm{C} & -9.78842595 & -2.69114776 & -0.41720884 \\ \mathrm{C} & -9.02693682 & -1.50513453 & -0.14424353 \\ \mathrm{C} & -9.71830668 & -0.25642847 & -0.04177310\end{array}$




\begin{tabular}{|c|c|c|c|}
\hline $\mathrm{C}$ & -7.66484961 & 1.29624518 & -0.19307336 \\
\hline $\mathrm{C}$ & -7.08142608 & 0.85191720 & -1.38689925 \\
\hline $\mathrm{H}$ & -7.68918562 & 0.32628001 & -2.11597489 \\
\hline $\mathrm{C}$ & -5.74369372 & 1.08434782 & -1.65270583 \\
\hline $\mathrm{H}$ & -5.31396216 & 0.73652179 & -2.58629869 \\
\hline $\mathrm{C}$ & -4.93544612 & 1.76481576 & -0.73506131 \\
\hline $\mathrm{C}$ & -5.53058373 & 2.25089089 & 0.43532470 \\
\hline $\mathrm{H}$ & -4.91972895 & 2.77132425 & 1.16567945 \\
\hline $\mathrm{C}$ & -6.86854252 & 2.02972501 & 0.70012437 \\
\hline $\mathrm{H}$ & -7.29154406 & 2.38627456 & 1.63104295 \\
\hline $\mathrm{C}$ & -3.47787343 & 1.90937745 & -0.94454179 \\
\hline $\mathrm{C}$ & -2.81666702 & 3.11646130 & -0.70206868 \\
\hline $\mathrm{H}$ & -3.39088518 & 4.00219291 & -0.44747958 \\
\hline $\mathrm{C}$ & -1.43675576 & 3.20317010 & -0.80289154 \\
\hline $\mathrm{H}$ & -0.95698128 & 4.16218162 & -0.63869403 \\
\hline $\mathrm{C}$ & -0.66123834 & 2.08067753 & -1.11647388 \\
\hline $\mathrm{C}$ & -1.33093510 & 0.88172011 & -1.38244019 \\
\hline $\mathrm{H}$ & -0.76491830 & -0.01908420 & -1.59281296 \\
\hline $\mathrm{C}$ & -2.71262986 & 0.80071634 & -1.31382494 \\
\hline $\mathrm{H}$ & -3.19907632 & -0.15478273 & -1.48146523 \\
\hline $\mathrm{C}$ & 0.82125913 & 2.12980156 & -1.09880462 \\
\hline $\mathrm{C}$ & 1.59252465 & 1.25166658 & -1.86716109 \\
\hline $\mathrm{H}$ & 1.10724689 & 0.57253770 & -2.55985311 \\
\hline $\mathrm{C}$ & 2.97291636 & 1.21688560 & -1.75200996 \\
\hline $\mathrm{H}$ & 3.52517270 & 0.47950246 & -2.32464947 \\
\hline $\mathrm{C}$ & 3.65077475 & 2.07369460 & -0.87860178 \\
\hline $\mathrm{C}$ & 2.88416314 & 2.99578475 & -0.15770149 \\
\hline $\mathrm{H}$ & 3.36933759 & 3.70936784 & 0.49964273 \\
\hline $\mathrm{C}$ & 1.50257412 & 3.01578081 & -0.25738327 \\
\hline $\mathrm{H}$ & 0.94460970 & 3.71391892 & 0.35731856 \\
\hline $\mathrm{C}$ & 5.11210301 & 1.95117999 & -0.66327042 \\
\hline $\mathrm{C}$ & 5.96404773 & 1.41941922 & -1.63881325 \\
\hline $\mathrm{H}$ & 5.57403982 & 1.17498197 & -2.62068100 \\
\hline $\mathrm{C}$ & 7.30847895 & 1.20325006 & -1.38007175 \\
\hline $\mathrm{H}$ & 7.93998780 & 0.77912949 & -2.15456261 \\
\hline $\mathrm{C}$ & 7.86151595 & 1.54017165 & -0.14240066 \\
\hline $\mathrm{C}$ & 7.03037355 & 2.13449269 & 0.81242706 \\
\hline $\mathrm{H}$ & 7.43374652 & 2.41585441 & 1.77865699 \\
\hline $\mathrm{C}$ & 5.68309298 & 2.31392893 & 0.56376958 \\
\hline $\mathrm{H}$ & 5.05698585 & 2.71343228 & 1.35357937 \\
\hline $\mathrm{C}$ & -9.98919685 & 4.55778510 & 0.72847975 \\
\hline $\mathrm{H}$ & -10.57379637 & 4.46885246 & 1.64998774 \\
\hline
\end{tabular}




\begin{tabular}{|c|c|c|c|}
\hline $\mathrm{H}$ & -10.67348940 & 4.71062545 & -0.11248506 \\
\hline $\mathrm{C}$ & -8.96750005 & 5.66584619 & 0.82166945 \\
\hline $\mathrm{H}$ & -8.37852826 & 5.72737130 & -0.09618840 \\
\hline $\mathrm{H}$ & -9.47802894 & 6.62075411 & 0.97057097 \\
\hline $\mathrm{H}$ & -8.29123285 & 5.50260448 & 1.66413854 \\
\hline $\mathrm{H}$ & 9.22102797 & 5.60777185 & 0.84698076 \\
\hline $\mathrm{C}$ & -9.73615138 & -5.05792323 & -0.74750672 \\
\hline $\mathrm{H}$ & -10.28667072 & -5.05105448 & -1.69260061 \\
\hline $\mathrm{H}$ & -10.41045465 & -5.28886681 & 0.08207051 \\
\hline $\mathrm{H}$ & -8.94041607 & -5.79898603 & -0.79051081 \\
\hline $\mathrm{H}$ & 9.49712520 & -5.48435697 & -0.90789192 \\
\hline $\mathrm{O}$ & 9.53340585 & -3.49892627 & -0.59071088 \\
\hline $\mathrm{O}$ & 9.35823940 & 3.62350800 & 0.55388518 \\
\hline $\mathrm{C}$ & 9.35571088 & -1.20776957 & -0.13046874 \\
\hline $\mathrm{C}$ & 10.17945184 & -2.34930465 & -0.43900653 \\
\hline $\mathrm{C}$ & 11.57022152 & -2.26125635 & -0.50318575 \\
\hline $\mathrm{H}$ & 12.17318867 & -3.13544685 & -0.70588230 \\
\hline $\mathrm{C}$ & 12.17263087 & -1.05220350 & -0.24622872 \\
\hline $\mathrm{H}$ & 13.25620664 & -0.98375656 & -0.25084775 \\
\hline $\mathrm{C}$ & 11.42818273 & 0.10834167 & 0.01578575 \\
\hline $\mathrm{C}$ & 12.11866146 & 1.30159789 & 0.27249429 \\
\hline $\mathrm{H}$ & 13.20401606 & 1.28183803 & 0.28872238 \\
\hline $\mathrm{C}$ & 11.45811929 & 2.48536218 & 0.50277822 \\
\hline $\mathrm{H}$ & 12.01704074 & 3.38967877 & 0.70021356 \\
\hline $\mathrm{C}$ & 10.06511347 & 2.50598416 & 0.42038222 \\
\hline $\mathrm{C}$ & 9.30111641 & 1.32482178 & 0.12936103 \\
\hline $\mathrm{C}$ & 9.99104501 & 0.07646692 & 0.00817718 \\
\hline $\mathrm{C}$ & 7.93676019 & -1.47640980 & 0.15327463 \\
\hline $\mathrm{C}$ & 7.35979273 & -1.04089078 & 1.35351085 \\
\hline $\mathrm{H}$ & 7.97150422 & -0.52101889 & 2.08345031 \\
\hline $\mathrm{C}$ & 6.02336480 & -1.27459199 & 1.62445408 \\
\hline $\mathrm{H}$ & 5.59853162 & -0.93353896 & 2.56278137 \\
\hline $\mathrm{C}$ & 5.21031143 & -1.94883613 & 0.70634403 \\
\hline $\mathrm{C}$ & 5.79942022 & -2.42721685 & -0.47016095 \\
\hline $\mathrm{H}$ & 5.18503871 & -2.94344297 & -1.20052368 \\
\hline $\mathrm{C}$ & 7.13581201 & -2.20368227 & -0.74079767 \\
\hline $\mathrm{H}$ & 7.55323951 & -2.55351163 & -1.67680591 \\
\hline $\mathrm{C}$ & 3.75370001 & -2.09419730 & 0.92127787 \\
\hline $\mathrm{C}$ & 3.09140514 & -3.30014816 & 0.67621370 \\
\hline $\mathrm{H}$ & 3.66460078 & -4.18514969 & 0.41684183 \\
\hline $\mathrm{C}$ & 1.71173700 & -3.38650767 & 0.78026902 \\
\hline $\mathrm{H}$ & 1.23120991 & -4.34473460 & 0.61385006 \\
\hline
\end{tabular}




\begin{tabular}{lrrr}
$\mathrm{C}$ & 0.93739166 & -2.26465184 & 1.09913400 \\
$\mathrm{C}$ & 1.60842609 & -1.06714274 & 1.36830037 \\
$\mathrm{H}$ & 1.04341271 & -0.16680202 & 1.58326396 \\
$\mathrm{C}$ & 2.99001054 & -0.98666079 & 1.29702658 \\
$\mathrm{H}$ & 3.47742396 & -0.03210377 & 1.46737275 \\
$\mathrm{C}$ & -0.54519569 & -2.31254108 & 1.08279528 \\
$\mathrm{C}$ & -1.31530672 & -1.43401894 & 1.85182922 \\
$\mathrm{H}$ & -0.82924032 & -0.75595337 & 2.54502229 \\
$\mathrm{C}$ & -2.69568357 & -1.39723395 & 1.73667980 \\
$\mathrm{H}$ & -3.24682493 & -0.65940801 & 2.30982453 \\
$\mathrm{C}$ & -3.37491829 & -2.25267607 & 0.86308776 \\
$\mathrm{C}$ & -2.60954510 & -3.17563989 & 0.14210379 \\
$\mathrm{H}$ & -3.09565444 & -3.88859868 & -0.51520811 \\
$\mathrm{C}$ & -1.22790659 & -3.19725064 & 0.24123718 \\
$\mathrm{H}$ & -0.67117211 & -3.89584757 & -0.37405152 \\
$\mathrm{C}$ & -4.83638871 & -2.12834565 & 0.64775066 \\
$\mathrm{C}$ & -5.68738635 & -1.59419813 & 1.62242967 \\
$\mathrm{H}$ & -5.29688720 & -1.34805715 & 2.60370335 \\
$\mathrm{C}$ & -7.03226176 & -1.37812497 & 1.36404649 \\
$\mathrm{H}$ & -7.66323339 & -0.95262084 & 2.13826818 \\
$\mathrm{C}$ & -7.58553668 & -1.71633890 & 0.12733947 \\
$\mathrm{C}$ & -6.75537812 & -2.31211611 & -0.82685050 \\
$\mathrm{H}$ & -7.15999197 & -2.59610555 & -1.79192745 \\
$\mathrm{C}$ & -5.40802882 & -2.49243174 & -0.57846817 \\
$\mathrm{H}$ & -4.78278172 & -2.89446255 & -1.36770223 \\
$\mathrm{C}$ & 10.25496830 & -4.70715568 & -0.83273521 \\
$\mathrm{H}$ & 10.81638397 & -4.64451622 & -1.76897203 \\
$\mathrm{H}$ & 10.92798693 & -4.92660919 & 0.00062658 \\
$\mathrm{H}$ & 10.01647446 & 4.86744855 & 0.78930788 \\
& 10.56948051 & 4.84392431 & 1.73258778 \\
$\mathrm{H}$ & & & -0.03832667 \\
\hline
\end{tabular}

Cartesian coordinates of $\mathbf{N P - 2} \mathbf{2}^{2+}$ (triplet, optimized at UCAM/6-31G(d), $\mathrm{OC}_{8} \mathrm{H}_{17}$ is replaced by $\mathrm{OCH}_{3}$ )

$\begin{array}{lrrr}\mathrm{O} & 9.26908928 & 3.32773763 & -0.51373617 \\ \mathrm{O} & 9.07876084 & -3.81035717 & 0.53039072 \\ \mathrm{C} & 9.08549181 & 1.03053468 & -0.08447234 \\ \mathrm{C} & 9.91154898 & 2.17781469 & -0.37412543 \\ \mathrm{C} & 11.30391429 & 2.08742766 & -0.43200383 \\ \mathrm{H} & 11.90908716 & 2.96271336 & -0.62150386 \\ \mathrm{C} & 11.90217965 & 0.87493276 & -0.18772296 \\ \mathrm{H} & 12.98569187 & 0.80452431 & -0.18875650 \\ \mathrm{C} & 11.15541038 & -0.28972587 & 0.05561690\end{array}$




\begin{tabular}{|c|c|c|c|}
\hline $\mathrm{C}$ & 11.84358343 & -1.48630237 & 0.29606626 \\
\hline $\mathrm{H}$ & 12.92887717 & -1.46811135 & 0.31704199 \\
\hline $\mathrm{C}$ & 11.18053005 & -2.67359657 & 0.50385152 \\
\hline $\mathrm{H}$ & 11.73800082 & -3.58154174 & 0.68844878 \\
\hline $\mathrm{C}$ & 9.78825248 & -2.69136088 & 0.41653937 \\
\hline $\mathrm{C}$ & 9.02693581 & -1.50512112 & 0.14419556 \\
\hline $\mathrm{C}$ & 9.71846570 & -0.25655210 & 0.04211499 \\
\hline $\mathrm{C}$ & 7.66511726 & 1.29625385 & 0.19321953 \\
\hline $\mathrm{C}$ & 7.08162786 & 0.85245033 & 1.38718088 \\
\hline $\mathrm{H}$ & 7.68931305 & 0.32706295 & 2.11650347 \\
\hline $\mathrm{C}$ & 5.74388407 & 1.08512132 & 1.65280524 \\
\hline $\mathrm{H}$ & 5.31402725 & 0.73765991 & 2.58647513 \\
\hline $\mathrm{C}$ & 4.93574220 & 1.76510955 & 0.73476289 \\
\hline $\mathrm{C}$ & 5.53093722 & 2.25062255 & -0.43581177 \\
\hline $\mathrm{H}$ & 4.92012598 & 2.77072286 & -1.16643926 \\
\hline $\mathrm{C}$ & 6.86894930 & 2.02940557 & -0.70036448 \\
\hline $\mathrm{H}$ & 7.29207424 & 2.38560732 & -1.63136747 \\
\hline $\mathrm{C}$ & 3.47806314 & 1.90964253 & 0.94405566 \\
\hline $\mathrm{C}$ & 2.81698692 & 3.11693191 & 0.70242758 \\
\hline $\mathrm{H}$ & 3.39128227 & 4.00282241 & 0.44855105 \\
\hline $\mathrm{C}$ & 1.43703355 & 3.20363377 & 0.80316122 \\
\hline $\mathrm{H}$ & 0.95729650 & 4.16277843 & 0.63960152 \\
\hline $\mathrm{C}$ & 0.66146470 & 2.08096045 & 1.11581717 \\
\hline $\mathrm{C}$ & 1.33102774 & 0.88177391 & 1.38085784 \\
\hline $\mathrm{H}$ & 0.76492591 & -0.01914796 & 1.59052017 \\
\hline $\mathrm{C}$ & 2.71274879 & 0.80074093 & 1.31231567 \\
\hline $\mathrm{H}$ & 3.19912542 & -0.15490846 & 1.47930852 \\
\hline $\mathrm{C}$ & -0.82110180 & 2.13014624 & 1.09817085 \\
\hline $\mathrm{C}$ & -1.59229824 & 1.25212561 & 1.86665935 \\
\hline $\mathrm{H}$ & -1.10696107 & 0.57306932 & 2.55938833 \\
\hline $\mathrm{C}$ & -2.97271463 & 1.21728182 & 1.75152651 \\
\hline $\mathrm{H}$ & -3.52496854 & 0.47993707 & 2.32422974 \\
\hline $\mathrm{C}$ & -3.65057607 & 2.07399594 & 0.87809538 \\
\hline $\mathrm{C}$ & -2.88404389 & 2.99600168 & 0.15707381 \\
\hline $\mathrm{H}$ & -3.36928496 & 3.70948332 & -0.50034362 \\
\hline $\mathrm{C}$ & -1.50241253 & 3.01598724 & 0.25665968 \\
\hline $\mathrm{H}$ & -0.94444788 & 3.71402447 & -0.35816746 \\
\hline $\mathrm{C}$ & -5.11198673 & 1.95138188 & 0.66281253 \\
\hline $\mathrm{C}$ & -5.96395047 & 1.42062047 & 1.63880361 \\
\hline $\mathrm{H}$ & -5.57393663 & 1.17701807 & 2.62088235 \\
\hline $\mathrm{C}$ & -7.30843735 & 1.20431570 & 1.38022674 \\
\hline $\mathrm{H}$ & -7.94002410 & 0.78102674 & 2.15511881 \\
\hline
\end{tabular}




\begin{tabular}{|c|c|c|c|}
\hline $\mathrm{C}$ & -7.86131327 & 1.53995765 & 0.14219555 \\
\hline $\mathrm{C}$ & -7.03015426 & 2.13326881 & -0.81316550 \\
\hline $\mathrm{H}$ & -7.43352565 & 2.41384488 & -1.77963987 \\
\hline $\mathrm{C}$ & -5.68285623 & 2.31296323 & -0.56458064 \\
\hline $\mathrm{H}$ & -5.05667270 & 2.71179717 & -1.35467684 \\
\hline $\mathrm{C}$ & 9.98953071 & 4.55771479 & -0.72808173 \\
\hline $\mathrm{H}$ & 10.57424638 & 4.46874369 & -1.64951149 \\
\hline $\mathrm{H}$ & 10.67371350 & 4.71063422 & 0.11295863 \\
\hline $\mathrm{C}$ & 8.96778734 & 5.66571872 & -0.82146417 \\
\hline $\mathrm{H}$ & 8.37867712 & 5.72724351 & 0.09630481 \\
\hline $\mathrm{H}$ & 9.47829797 & 6.62064425 & -0.97031187 \\
\hline $\mathrm{H}$ & 8.29165418 & 5.50242472 & -1.66402877 \\
\hline $\mathrm{H}$ & -9.21996485 & 5.60782985 & -0.84575572 \\
\hline $\mathrm{C}$ & 9.73538482 & -5.05822012 & 0.74606181 \\
\hline $\mathrm{H}$ & 10.28592321 & -5.05177527 & 1.69114776 \\
\hline $\mathrm{H}$ & 10.40961701 & -5.28907750 & -0.08359880 \\
\hline $\mathrm{H}$ & 8.93945974 & -5.79908948 & 0.78885572 \\
\hline $\mathrm{H}$ & -9.49749520 & -5.48434083 & 0.90697459 \\
\hline $\mathrm{O}$ & -9.53381565 & -3.49886333 & 0.59005342 \\
\hline $\mathrm{O}$ & -9.35775446 & 3.62355153 & -0.55304528 \\
\hline $\mathrm{C}$ & -9.35601299 & -1.20770898 & 0.13005299 \\
\hline $\mathrm{C}$ & -10.17986344 & -2.34926257 & 0.43844532 \\
\hline $\mathrm{C}$ & -11.57060777 & -2.26108862 & 0.50258180 \\
\hline $\mathrm{H}$ & -12.17367642 & -3.13523242 & 0.70517770 \\
\hline $\mathrm{C}$ & -12.17288921 & -1.05190128 & 0.24582382 \\
\hline $\mathrm{H}$ & -13.25645895 & -0.98334224 & 0.25058197 \\
\hline $\mathrm{C}$ & -11.42836482 & 0.10864276 & -0.01601585 \\
\hline $\mathrm{C}$ & -12.11868278 & 1.30207783 & -0.27225003 \\
\hline $\mathrm{H}$ & -13.20404199 & 1.28249092 & -0.28843629 \\
\hline $\mathrm{C}$ & -11.45795863 & 2.48585193 & -0.50206889 \\
\hline $\mathrm{H}$ & -12.01675748 & 3.39032206 & -0.69915444 \\
\hline $\mathrm{C}$ & -10.06495117 & 2.50622812 & -0.41973027 \\
\hline $\mathrm{C}$ & -9.30112107 & 1.32482490 & -0.12931671 \\
\hline $\mathrm{C}$ & -9.99121704 & 0.07660236 & -0.00849501 \\
\hline $\mathrm{C}$ & -7.93704156 & -1.47639787 & -0.15345526 \\
\hline $\mathrm{C}$ & -7.35999816 & -1.04148139 & -1.35384328 \\
\hline $\mathrm{H}$ & -7.97163482 & -0.52193930 & -2.08408504 \\
\hline $\mathrm{C}$ & -6.02355292 & -1.27543154 & -1.62457496 \\
\hline $\mathrm{H}$ & -5.59858417 & -0.93478956 & -2.56298942 \\
\hline $\mathrm{C}$ & -5.21061082 & -1.94913183 & -0.70601860 \\
\hline $\mathrm{C}$ & -5.79979090 & -2.42688348 & 0.47069497 \\
\hline $\mathrm{H}$ & -5.18545687 & -2.94273545 & 1.20136155 \\
\hline
\end{tabular}




\begin{tabular}{|c|c|c|c|}
\hline $\mathrm{C}$ & -7.13623826 & -2.20328619 & 0.74105594 \\
\hline $\mathrm{H}$ & -7.55379906 & -2.55270988 & 1.67716389 \\
\hline $\mathrm{C}$ & -3.75389356 & -2.09446779 & -0.92073379 \\
\hline $\mathrm{C}$ & -3.09173120 & -3.30060705 & -0.67641539 \\
\hline $\mathrm{H}$ & -3.66500600 & -4.18575036 & -0.41768813 \\
\hline $\mathrm{C}$ & -1.71201969 & -3.38696722 & -0.78037286 \\
\hline $\mathrm{H}$ & -1.23153481 & -4.34531922 & -0.61452656 \\
\hline $\mathrm{C}$ & -0.93762302 & -2.26495350 & -1.09840677 \\
\hline $\mathrm{C}$ & -1.60852580 & -1.06723011 & -1.36675324 \\
\hline $\mathrm{H}$ & -1.04342142 & -0.16679154 & -1.58108807 \\
\hline $\mathrm{C}$ & -2.99013057 & -0.98671263 & -1.29555474 \\
\hline $\mathrm{H}$ & -3.47748050 & -0.03202111 & -1.46534038 \\
\hline $\mathrm{C}$ & 0.54502935 & -2.31289796 & -1.08210387 \\
\hline $\mathrm{C}$ & 1.31506126 & -1.43454278 & -1.85134198 \\
\hline $\mathrm{H}$ & 0.82892454 & -0.75660709 & -2.54461957 \\
\hline $\mathrm{C}$ & 2.69546171 & -1.39769024 & -1.73623227 \\
\hline $\mathrm{H}$ & 3.24659464 & -0.65994567 & -2.30949883 \\
\hline $\mathrm{C}$ & 3.37470997 & -2.25297021 & -0.86255434 \\
\hline $\mathrm{C}$ & 2.60942145 & -3.17579196 & -0.14136256 \\
\hline $\mathrm{H}$ & 3.09560748 & -3.88859465 & 0.51607286 \\
\hline $\mathrm{C}$ & 1.22774472 & -3.19740744 & -0.24039179 \\
\hline $\mathrm{H}$ & 0.67101077 & -3.89585123 & 0.37508092 \\
\hline $\mathrm{C}$ & 4.83626166 & -2.12852616 & -0.64729006 \\
\hline $\mathrm{C}$ & 5.68727438 & -1.59539151 & -1.62243224 \\
\hline $\mathrm{H}$ & 5.29677480 & -1.35010084 & -2.60392352 \\
\hline $\mathrm{C}$ & 7.03221016 & -1.37918737 & -1.36422696 \\
\hline $\mathrm{H}$ & 7.66325001 & -0.95451548 & -2.13885815 \\
\hline $\mathrm{C}$ & 7.58533072 & -1.71612780 & -0.12716421 \\
\hline $\mathrm{C}$ & 6.75515798 & -2.31087214 & 0.82757995 \\
\hline $\mathrm{H}$ & 7.15978257 & -2.59405731 & 1.79290604 \\
\hline $\mathrm{C}$ & 5.40779328 & -2.49143573 & 0.57928777 \\
\hline $\mathrm{H}$ & 4.78247651 & -2.89278584 & 1.36882059 \\
\hline $\mathrm{C}$ & -10.25535851 & -4.70715046 & 0.83193723 \\
\hline $\mathrm{H}$ & -10.81675218 & -4.64462459 & 1.76819233 \\
\hline $\mathrm{H}$ & -10.92838640 & -4.92648478 & -0.00144666 \\
\hline $\mathrm{C}$ & -10.01564021 & 4.86775361 & -0.78810359 \\
\hline $\mathrm{H}$ & -10.56880507 & 4.84458448 & -1.73129793 \\
\hline $\mathrm{H}$ & -10.68731214 & 5.11202194 & 0.03969469 \\
\hline
\end{tabular}


Chemical structures used for strain energy calculation.
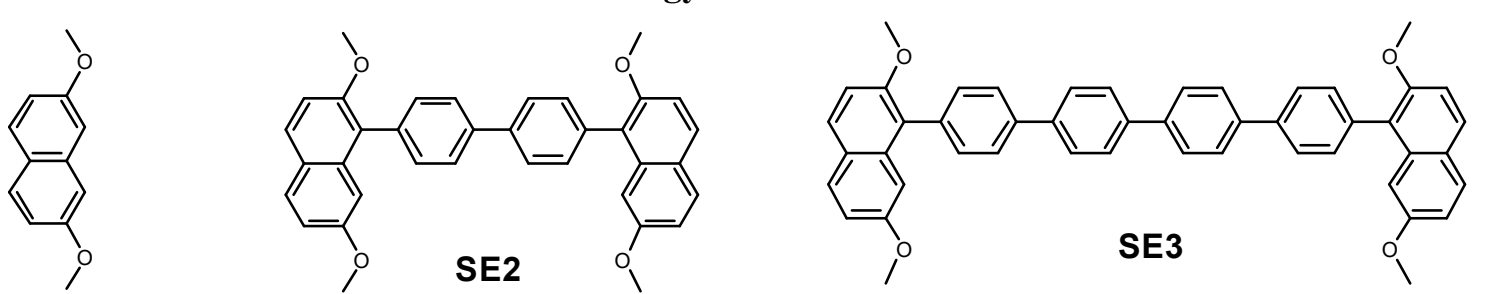

SE1
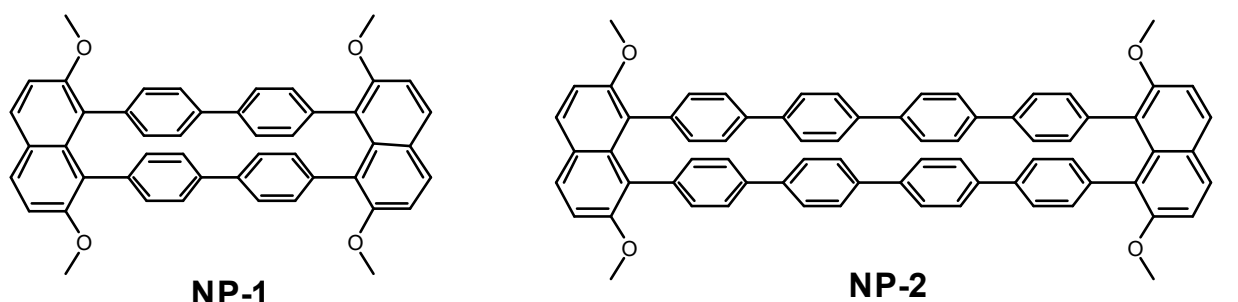

Cartesian coordinates of SE1 (optimized at RB3LYP/6-31g(d,p))

$\mathrm{C}$

$\mathrm{C}$

$\mathrm{C}$

$\mathrm{C}$

$\mathrm{C}$

$\mathrm{C}$

$\mathrm{C}$

$\mathrm{C}$

$\mathrm{C}$

$\mathrm{C}$

$\mathrm{O}$

$\mathrm{C}$

$\mathrm{O}$

$\mathrm{C}$

$\mathrm{H}$

$\mathrm{H}$

$\mathrm{H}$

$\mathrm{H}$

$\mathrm{H}$

$\mathrm{H}$

$\mathrm{H}$

$\mathrm{H}$

$\mathrm{H}$

$\mathrm{H}$

$\mathrm{H}$

$\mathrm{H}$

$$
-2.43370560
$$

$-1.24599667$

$-0.00001761$

$-0.00001276$

$-1.24107695$

$-2.43242934$

1.24595449

2.43366898

2.43240813

1.24105134

$-3.67483275$

$-3.76312536$

3.67483084

3.76323441

$-3.39037323$

$-1.24783586$

$-1.22642669$

1.24779076

3.39033014

1.22640125

$-4.82805350$

$-3.29569334$

$-3.29574634$

4.82817957

3.29590101

3.29582316
1.39900109

2.08524467

1.40293389

$-0.02695345$

$-0.72208204$

$-0.02198748$

2.08524785

1.39900843

$-0.02197663$

$-0.72207626$

$-0.58837419$

$-2.00377811$

$-0.58833194$

$-2.00373277$

1.91024628

3.17218956

$-1.80591430$

3.17219276

1.91026535

$-1.80590473$

$-2.24066368$

$-2.43781316$

$-2.43785379$

$-2.24054345$

$-2.43786227$

$-2.43778462$
0.00000774

$-0.00002878$

$-0.00001526$

0.00000994

0.00004745

0.00005209

$-0.00001699$

0.00000332

0.00001507

0.00002003

0.00000447

$-0.00003791$

0.00002491

$-0.00003802$

$-0.00001721$

$-0.00006479$

0.00006522

$-0.00003617$

0.00000215

0.00003925

$-0.00010236$

$-0.89331478$

0.89324679

$-0.00009319$

0.89324406

$-0.89331621$

Cartesian coordinates of SE2 (optimized at RB3LYP/6-31g(d,p)) 
C

$\mathrm{C}$

C

C

C

C

C

C

C

C

C

C

C

C

C

C

$\mathrm{O}$

C

$\mathrm{O}$

$\mathrm{C}$

C

C

C

C

C

C

C

C

C

C

$\mathrm{O}$

$\mathrm{O}$

C

C

C

C

C

C

C

C

$\mathrm{H}$

$\mathrm{H}$

$$
-7.26034165
$$

$-7.98848949$

$-7.33717009$

$-5.90471860$

$-5.15441959$

$-5.84820612$

$-8.07285236$

$-7.44299146$

$-6.02354530$

$-5.27237605$

$-3.66440582$

$-2.99334037$

$-1.60276356$

$-0.82307299$

$-1.49754284$

$-2.88670023$

$-5.16551481$

$-4.91187152$

$-5.50892318$

$-4.09693223$

7.42637879

8.00172612

7.22169917

5.78913439

5.22060216

6.01356227

7.82260629

7.04748338

5.63484101

4.98814400

5.34892797

4.90869807

6.10537404

4.64067957

0.65837583

1.45108077

2.84149099

3.49928001

2.70826748

1.31928031

$-7.74044189$

$-9.07453060$
$-1.94789726$

$-0.61337357$

$-0.80943477 \quad-0.36622137$

$0.41437114 \quad-0.07528593$

$0.46343777-0.03448040$

$-0.74063151 \quad-0.26593893$

$-1.90803850 \quad-0.56471198$

1.60904329

2.79913865

2.85506207

1.71679887

$-0.75889361$

0.16171951

0.41157918

0.42651023

0.20854998

$-0.42695526$

$-0.19190529$

0.99634719

$-0.45362246$

1.07094361

$-0.81693404$

$-0.03944909$

$-1.15500134$

$-1.22525827$

$-1.12779653$

$-1.30052031$

$-3.07549697$

$-0.85268180$

$-3.90636851$

0.27946194

4.09654287

4.24278701

2.52258996

0.66225269

0.67887914

$-0.34483352$

1.29266673

$-0.11581219$

0.12960393

0.09488566

0.23863959

0.05502844

1.51672925

2.63387685

$-0.16232034$

$-0.36039743$

$-1.12854374$

0.36003521

$-2.23804592$

0.58135408

$-2.13905584$

$-0.94134040$

0.53371917

0.26196222

3.81197495

$-0.56051796$

$-3.28627623$

0.79400115

4.99611134

$-0.75394614$

$-4.08858752$

$-0.35553991$

$-0.84521204$

0.03752064

$-0.46430069$

$-1.05752942$

$-0.49116476$

$-0.98376030$

$-0.89775901$

0.18850482

$-1.28321850$

1.28215885

$-1.25610957$

1.20779883

$-2.89022970$

$-0.85661308$

$-0.83160787$

$-0.40131029$ 


$\begin{array}{lrrr}\mathrm{H} & -9.15843160 & 1.56389074 & 0.13592739 \\ \mathrm{H} & -7.99644467 & 3.71471867 & 0.59092729 \\ \mathrm{H} & -4.19236546 & 1.76072720 & 0.20725526 \\ \mathrm{H} & -3.57100336 & -0.14195682 & 1.87097463 \\ \mathrm{H} & -1.11433473 & -0.16858618 & 1.99799425 \\ \mathrm{H} & -0.92577104 & -1.46836297 & -2.09357694 \\ \mathrm{H} & -3.38162567 & -1.40782628 & -2.22426174 \\ \mathrm{H} & -4.40140942 & -4.79596971 & -0.09503262 \\ \mathrm{H} & -5.84636639 & -4.20524303 & 0.77259442 \\ \mathrm{H} & -4.26863521 & -3.39979860 & 1.00925899 \\ \mathrm{H} & -3.90541817 & 5.29734256 & 0.88193547 \\ \mathrm{H} & -3.63692489 & 3.63205327 & 1.46590065 \\ \mathrm{H} & -3.65238618 & 3.97197509 & -0.28698466 \\ \mathrm{H} & 8.05508645 & 3.39056382 & -0.50203077 \\ \mathrm{H} & 9.08463661 & 1.20314073 & -0.08978422 \\ \mathrm{H} & 4.14590723 & 1.64794798 & -0.16916365 \\ \mathrm{H} & 8.90674706 & -1.19655768 & 0.39490295 \\ \mathrm{H} & 7.48659964 & -3.20524269 & 0.80371557 \\ \mathrm{H} & 5.37737432 & 5.79804023 & -0.88550698 \\ \mathrm{H} & 6.73640230 & 5.22467475 & 0.11454929 \\ \mathrm{H} & 6.73690005 & 4.93632360 & -1.64975403 \\ \mathrm{H} & 5.57025323 & -4.40930224 & -0.84426190 \\ \mathrm{H} & 4.09748461 & -4.96741932 & -0.00241526 \\ \mathrm{H} & 4.02259594 & -3.54801206 & -1.08266242 \\ \mathrm{H} & 0.97403694 & -0.12200424 & -1.97098908 \\ \mathrm{H} & 3.42844400 & -0.18978443 & -1.84635531 \\ \mathrm{H} & 3.19207955 & -1.61914452 & 2.19294609 \\ \mathrm{H} & 0.73643871 & -1.58397547 & 2.06334214\end{array}$

Cartesian coordinates of SE3 (optimized at RB3LYP/6-31g(d,p))

$\begin{array}{cccc}\text { C } & -11.47540947 & -1.24715607 & -1.55533432 \\ \mathrm{C} & -12.20769161 & -0.41823691 & -0.73559209 \\ \mathrm{C} & -11.57607888 & 0.45475802 & 0.17697215 \\ \mathrm{C} & -10.14380901 & 0.47040827 & 0.25088821 \\ \mathrm{C} & -9.38693668 & -0.40305195 & -0.59148300 \\ \mathrm{C} & -10.06124079 & -1.23861459 & -1.48371411 \\ \mathrm{C} & -12.32168454 & 1.32535417 & 1.02095052 \\ \mathrm{C} & -11.70051671 & 2.18040157 & 1.89070370 \\ \mathrm{C} & -10.27990419 & 2.21039254 & 1.95355640 \\ \mathrm{C} & -9.51984214 & 1.37994128 & 1.15539941 \\ \mathrm{C} & -7.89579441 & -0.44078769 & -0.52275971 \\ \mathrm{C} & -7.23923547 & -0.91869807 & 0.62144922\end{array}$




\begin{tabular}{|c|c|c|c|}
\hline $\mathrm{C}$ & -5.84879176 & -0.94348236 & 0.69667180 \\
\hline $\mathrm{C}$ & -5.05615526 & -0.49297339 & -0.37175593 \\
\hline $\mathrm{C}$ & -5.71599563 & -0.02160701 & -1.51952416 \\
\hline $\mathrm{C}$ & -7.10544163 & 0.00339814 & -1.59308186 \\
\hline $\mathrm{O}$ & -9.27964076 & -2.04867606 & -2.26141946 \\
\hline $\mathrm{C}$ & -9.90327815 & -2.94206272 & -3.16892784 \\
\hline $\mathrm{O}$ & -9.77504999 & 3.11185917 & 2.84491295 \\
\hline $\mathrm{C}$ & -8.36442659 & 3.21168384 & 2.96795719 \\
\hline $\mathrm{C}$ & -3.57542662 & -0.50754589 & -0.28765663 \\
\hline $\mathrm{C}$ & -2.80329022 & 0.51632996 & -0.86225081 \\
\hline $\mathrm{C}$ & -1.41523688 & 0.51011096 & -0.77411595 \\
\hline $\mathrm{C}$ & -0.73164334 & -0.52058139 & -0.10703826 \\
\hline $\mathrm{C}$ & -1.50338473 & -1.54700201 & 0.46378050 \\
\hline $\mathrm{C}$ & -2.89138903 & -1.54073789 & 0.37513519 \\
\hline $\mathrm{C}$ & 11.86057018 & 2.24924714 & -1.53196565 \\
\hline $\mathrm{C}$ & 12.42730477 & 1.31038629 & -0.71169804 \\
\hline $\mathrm{C}$ & 11.63046226 & 0.39836504 & 0.03523701 \\
\hline $\mathrm{C}$ & 10.20357016 & 0.46163249 & -0.09053443 \\
\hline $\mathrm{C}$ & 9.63705120 & 1.45764564 & -0.93714079 \\
\hline $\mathrm{C}$ & 10.44630826 & 2.32847706 & -1.64000871 \\
\hline $\mathrm{C}$ & 12.21539474 & -0.55194937 & 0.90784743 \\
\hline $\mathrm{C}$ & 11.42847785 & -1.40937511 & 1.63796176 \\
\hline $\mathrm{C}$ & 10.02142560 & -1.35619347 & 1.51304039 \\
\hline $\mathrm{C}$ & 9.39205795 & -0.45812990 & 0.65855823 \\
\hline $\mathrm{O}$ & 9.99722802 & 3.31418422 & -2.46975237 \\
\hline $\mathrm{O}$ & 9.27634197 & -2.21788046 & 2.29657424 \\
\hline $\mathrm{C}$ & 8.59499594 & 3.47064878 & -2.62443771 \\
\hline $\mathrm{C}$ & 9.01054402 & -3.48411649 & 1.69424253 \\
\hline $\mathrm{C}$ & 0.74730349 & -0.52131328 & -0.00393810 \\
\hline $\mathrm{C}$ & 1.39610877 & -0.98662545 & 1.15242971 \\
\hline $\mathrm{C}$ & 2.78312689 & -0.98140599 & 1.25381743 \\
\hline $\mathrm{C}$ & 3.58934025 & -0.50782607 & 0.20486585 \\
\hline $\mathrm{C}$ & 2.94067301 & -0.04499289 & -0.95241348 \\
\hline $\mathrm{C}$ & 1.55356119 & -0.05290543 & -1.05512010 \\
\hline $\mathrm{C}$ & 5.06817854 & -0.49451124 & 0.31759381 \\
\hline $\mathrm{C}$ & 5.88667858 & -0.75681722 & -0.79357829 \\
\hline $\mathrm{C}$ & 7.27514046 & -0.74033796 & -0.68433417 \\
\hline $\mathrm{C}$ & 7.90488816 & -0.46270950 & 0.53994707 \\
\hline $\mathrm{C}$ & 7.08816515 & -0.20745338 & 1.65237403 \\
\hline $\mathrm{C}$ & 5.70127387 & -0.21969979 & 1.54207790 \\
\hline $\mathrm{H}$ & -11.98572388 & -1.90656544 & -2.24618144 \\
\hline $\mathrm{H}$ & -13.29325646 & -0.42856344 & -0.78838901 \\
\hline
\end{tabular}




$\begin{array}{lrrr}\mathrm{H} & -13.40679313 & 1.30307453 & 0.96169681 \\ \mathrm{H} & -12.25874716 & 2.84878811 & 2.53738540 \\ \mathrm{H} & -8.44008935 & 1.40946870 & 1.19675063 \\ \mathrm{H} & -7.82878136 & -1.26937857 & 1.46358979 \\ \mathrm{H} & -5.37145722 & -1.29467316 & 1.60677097 \\ \mathrm{H} & -5.13182013 & 0.30801436 & -2.37366267 \\ \mathrm{H} & -7.58982587 & 0.36449824 & -2.49489851 \\ \mathrm{H} & -9.09131557 & -3.47783355 & -3.66298096 \\ \mathrm{H} & -10.54777075 & -3.66513262 & -2.65298314 \\ \mathrm{H} & -10.49476253 & -2.41109623 & -3.92580058 \\ \mathrm{H} & -8.18096036 & 3.97520682 & 3.72524958 \\ \mathrm{H} & -7.91974672 & 2.26274916 & 3.29312402 \\ \mathrm{H} & -7.89797012 & 3.51745520 & 2.02301696 \\ \mathrm{H} & -3.30045422 & 1.34796716 & -1.35247661 \\ \mathrm{H} & -0.85329776 & 1.33799065 & -1.19594247 \\ \mathrm{H} & -1.00664680 & -2.37894271 & 0.95398121 \\ \mathrm{H} & -3.45403519 & -2.36777265 & 0.79754778 \\ \mathrm{H} & 12.46073332 & 2.94983078 & -2.10268358 \\ \mathrm{H} & 13.50845237 & 1.25406301 & -0.61625992 \\ \mathrm{H} & 8.56062620 & 1.52516072 & -1.00881137 \\ \mathrm{H} & 13.29788681 & -0.58555685 & 0.99884354 \\ \mathrm{H} & 11.85743633 & -2.13112812 & 2.32548509 \\ \mathrm{H} & 8.45911132 & 4.29831962 & -3.32195785 \\ \mathrm{H} & 8.10879011 & 3.71500586 & -1.67159547 \\ \mathrm{H} & 8.13154716 & 2.56642553 & -3.03890780 \\ \mathrm{H} & 9.94130319 & -4.00995567 & 1.44351578 \\ \mathrm{H} & 8.40618925 & -3.37413271 & 0.78555171 \\ \mathrm{H} & 8.45345558 & -4.06820982 & 2.42955834 \\ \mathrm{H} & 0.80399201 & -1.34042368 & 1.99105308 \\ \mathrm{H} & 3.25084525 & -1.36448557 & 2.15574276 \\ \mathrm{H} & 3.53118014 & 0.34324729 & -1.77687038 \\ \mathrm{H} & 1.08732652 & 0.29573684 & -1.97175813 \\ \mathrm{H} & 5.43140967 & -1.00841436 & -1.74686782 \\ \mathrm{H} & 7.58367138 & -0.96180325 & -1.55645013 \\ \mathrm{H} & 0.00948939 & 2.60951161 \\ \mathrm{H} & 0.01399729 & 2.41425985\end{array}$

Cartesian coordinates of NP-1 (optimized at RB3LYP/6-31g(d,p))
$\mathrm{O}$
5.11478191
$-3.62968649$
0.16851004
$\mathrm{O}$
5.11479455
3.62968808
$-0.16850367$
O
$-5.11479593-3.62968030$
$-0.16885614$
$\mathrm{O}$
$-5.11480169$
3.62968538
0.16881072
C
5.03575593
$-1.28760365$
0.05066155 


\begin{tabular}{|c|c|c|c|}
\hline $\mathrm{C}$ & 5.80975330 & -2.45266349 & 0.10207091 \\
\hline $\mathrm{C}$ & 7.22343048 & -2.42586886 & 0.09851467 \\
\hline $\mathrm{H}$ & 7.79765886 & -3.34261361 & 0.13962509 \\
\hline $\mathrm{C}$ & 7.86004865 & -1.21465614 & 0.04750171 \\
\hline $\mathrm{H}$ & 8.94591227 & -1.17060385 & 0.04490750 \\
\hline $\mathrm{C}$ & 7.13790178 & -0.00000321 & -0.00001638 \\
\hline $\mathrm{C}$ & 7.86005225 & 1.21464752 & -0.04754107 \\
\hline $\mathrm{H}$ & 8.94591576 & 1.17059136 & -0.04496447 \\
\hline $\mathrm{C}$ & 7.22343757 & 2.42586257 & -0.09853870 \\
\hline $\mathrm{H}$ & 7.79766802 & 3.34260575 & -0.13965986 \\
\hline $\mathrm{C}$ & 5.80976060 & 2.45266175 & -0.10207444 \\
\hline $\mathrm{C}$ & 5.03575962 & 1.28760435 & -0.05066701 \\
\hline $\mathrm{C}$ & 5.69039494 & -0.00000091 & -0.00000827 \\
\hline $\mathrm{C}$ & 3.55363205 & -1.51411944 & 0.05520393 \\
\hline $\mathrm{C}$ & 2.90607422 & -2.01172466 & -1.08552967 \\
\hline $\mathrm{H}$ & 3.49064312 & -2.22295684 & -1.97635898 \\
\hline $\mathrm{C}$ & 1.53035422 & -2.21734538 & -1.10133685 \\
\hline $\mathrm{H}$ & 1.05767405 & -2.58917473 & -2.00596008 \\
\hline $\mathrm{C}$ & 0.74195169 & -1.93360763 & 0.02700967 \\
\hline $\mathrm{C}$ & 1.40739227 & -1.51860461 & 1.19197935 \\
\hline $\mathrm{H}$ & 0.83078159 & -1.28488020 & 2.08127158 \\
\hline $\mathrm{C}$ & 2.78511449 & -1.32029125 & 1.20754180 \\
\hline $\mathrm{H}$ & 3.26474063 & -0.96669392 & 2.11505996 \\
\hline $\mathrm{C}$ & -0.74195113 & -1.93362812 & -0.02733658 \\
\hline $\mathrm{C}$ & -1.53033485 & -2.21747942 & 1.10099607 \\
\hline $\mathrm{H}$ & -1.05763455 & -2.58937213 & 2.00558275 \\
\hline $\mathrm{C}$ & -2.90605469 & -2.01187306 & 1.08522684 \\
\hline $\mathrm{H}$ & -3.49060638 & -2.22317499 & 1.97605048 \\
\hline $\mathrm{C}$ & -3.55363348 & -1.51415493 & -0.05544789 \\
\hline $\mathrm{C}$ & -2.78514052 & -1.32024792 & -1.20778802 \\
\hline $\mathrm{H}$ & -3.26478106 & -0.96657241 & -2.11526737 \\
\hline $\mathrm{C}$ & -1.40741565 & -1.51855569 & -1.19226618 \\
\hline $\mathrm{H}$ & -0.83082349 & -1.28474765 & -2.08154831 \\
\hline $\mathrm{C}$ & 3.55363640 & 1.51412319 & -0.05520332 \\
\hline $\mathrm{C}$ & 2.78512335 & 1.32034052 & -1.20755255 \\
\hline $\mathrm{H}$ & 3.26475423 & 0.96677954 & -2.11508236 \\
\hline $\mathrm{C}$ & 1.40740213 & 1.51865764 & -1.19198940 \\
\hline $\mathrm{H}$ & 0.83079598 & 1.28496985 & -2.08129391 \\
\hline $\mathrm{C}$ & 0.74195514 & 1.93360966 & -0.02700434 \\
\hline $\mathrm{C}$ & 1.53035312 & 2.21729912 & 1.10135722 \\
\hline $\mathrm{H}$ & 1.05767040 & 2.58909141 & 2.00599418 \\
\hline $\mathrm{C}$ & 2.90607409 & 2.01168351 & 1.08554630 \\
\hline
\end{tabular}




\begin{tabular}{|c|c|c|c|}
\hline $\mathrm{H}$ & 3.49063844 & 2.22287952 & 1.97638720 \\
\hline $\mathrm{C}$ & -0.74194873 & 1.93362735 & 0.02733501 \\
\hline $\mathrm{C}$ & -1.40742061 & 1.51846662 & 1.19222991 \\
\hline $\mathrm{H}$ & -0.83083353 & 1.28458830 & 2.08149671 \\
\hline $\mathrm{C}$ & -2.78514547 & 1.32015832 & 1.20772884 \\
\hline $\mathrm{H}$ & -3.26479178 & 0.96641165 & 2.11517768 \\
\hline $\mathrm{C}$ & -3.55363249 & 1.51415599 & 0.05539930 \\
\hline $\mathrm{C}$ & -2.90604744 & 2.01196025 & -1.08523327 \\
\hline $\mathrm{H}$ & -3.49059394 & 2.22333330 & -1.97604343 \\
\hline $\mathrm{C}$ & -1.53032697 & 2.21756532 & -1.10097944 \\
\hline $\mathrm{H}$ & -1.05762327 & 2.58952908 & -2.00553494 \\
\hline $\mathrm{C}$ & -5.03574970 & -1.28760852 & -0.05082802 \\
\hline $\mathrm{C}$ & -5.80975917 & -2.45265873 & -0.10228799 \\
\hline $\mathrm{C}$ & -7.22343519 & -2.42585531 & -0.09870633 \\
\hline $\mathrm{H}$ & -7.79767013 & -3.34259271 & -0.13987524 \\
\hline $\mathrm{C}$ & -7.86004373 & -1.21464214 & -0.04761729 \\
\hline $\mathrm{H}$ & -8.94590703 & -1.17058158 & -0.04501322 \\
\hline $\mathrm{C}$ & -7.13788855 & 0.00000252 & -0.00002870 \\
\hline $\mathrm{C}$ & -7.86004320 & 1.21464683 & 0.04757956 \\
\hline $\mathrm{H}$ & -8.94590650 & 1.17058677 & 0.04499339 \\
\hline $\mathrm{C}$ & -7.22343397 & 2.42585937 & 0.09867303 \\
\hline $\mathrm{H}$ & -7.79766717 & 3.34259801 & 0.13985387 \\
\hline $\mathrm{C}$ & -5.80975896 & 2.45266131 & 0.10224728 \\
\hline $\mathrm{C}$ & -5.03574914 & 1.28761218 & 0.05075983 \\
\hline $\mathrm{C}$ & -5.69037970 & 0.00000255 & -0.00004481 \\
\hline $\mathrm{C}$ & 5.82754593 & -4.85286353 & 0.23032523 \\
\hline $\mathrm{H}$ & 6.46146366 & -4.91392846 & 1.12433514 \\
\hline $\mathrm{H}$ & 6.44856549 & -5.01252312 & -0.66061829 \\
\hline $\mathrm{H}$ & 5.06803580 & -5.63467210 & 0.27894919 \\
\hline $\mathrm{H}$ & 5.06806092 & 5.63468522 & -0.27873495 \\
\hline $\mathrm{H}$ & -5.06808016 & 5.63466591 & 0.27934263 \\
\hline $\mathrm{H}$ & -5.06804573 & -5.63467903 & -0.27904682 \\
\hline $\mathrm{C}$ & 5.82756604 & 4.85286697 & -0.23018906 \\
\hline $\mathrm{H}$ & 6.46148673 & 4.91402178 & -1.12419118 \\
\hline $\mathrm{H}$ & 6.44858428 & 5.01242889 & 0.66077251 \\
\hline $\mathrm{C}$ & -5.82755720 & -4.85286902 & -0.23046611 \\
\hline $\mathrm{H}$ & -6.46153605 & -4.91405387 & -1.12442528 \\
\hline $\mathrm{H}$ & -6.44851411 & -5.01241801 & 0.66054000 \\
\hline $\mathrm{C}$ & -5.82758054 & 4.85285126 & 0.23066339 \\
\hline $\mathrm{H}$ & -6.46152123 & 4.91386817 & 1.12466059 \\
\hline $\mathrm{H}$ & -6.44857999 & 5.01254485 & -0.66028791 \\
\hline
\end{tabular}


Cartesian coordinates of NP-2 (optimized at RB3LYP/6-31g(d,p))

\begin{tabular}{|c|c|c|c|}
\hline $\mathrm{O}$ & -9.38671268 & 3.63044724 & 0.23097864 \\
\hline $\mathrm{O}$ & -9.37634636 & -3.61396339 & -0.23398972 \\
\hline $\mathrm{C}$ & -9.30927103 & 1.28950506 & 0.06471445 \\
\hline $\mathrm{C}$ & -10.08016756 & 2.45237834 & 0.18003030 \\
\hline $\mathrm{C}$ & -11.49386765 & 2.42264211 & 0.21391066 \\
\hline $\mathrm{H}$ & -12.06663010 & 3.33705644 & 0.30073892 \\
\hline $\mathrm{C}$ & -12.13386080 & 1.21631802 & 0.10859831 \\
\hline $\mathrm{H}$ & -13.21980258 & 1.17462687 & 0.11137145 \\
\hline $\mathrm{C}$ & -11.41377513 & 0.00503633 & -0.00505555 \\
\hline $\mathrm{C}$ & -12.13109876 & -1.20777566 & -0.12125091 \\
\hline $\mathrm{H}$ & -13.21708843 & -1.16778858 & -0.12893312 \\
\hline $\mathrm{C}$ & -11.48888581 & -2.41322791 & -0.22315154 \\
\hline $\mathrm{H}$ & -12.05929930 & -3.32878562 & -0.31361118 \\
\hline $\mathrm{C}$ & -10.07532495 & -2.43922128 & -0.18207848 \\
\hline $\mathrm{C}$ & -9.30806535 & -1.27484528 & -0.06211524 \\
\hline $\mathrm{C}$ & -9.96730262 & 0.00692697 & -0.00158463 \\
\hline $\mathrm{C}$ & -7.83213031 & 1.51320010 & -0.05486573 \\
\hline $\mathrm{C}$ & -7.19029366 & 1.40920816 & -1.29495770 \\
\hline $\mathrm{H}$ & -7.76574386 & 1.11604663 & -2.16814758 \\
\hline $\mathrm{C}$ & -5.82780391 & 1.66496580 & -1.42199533 \\
\hline $\mathrm{H}$ & -5.35109474 & 1.56628272 & -2.39300365 \\
\hline $\mathrm{C}$ & -5.05903022 & 2.05722686 & -0.31578062 \\
\hline $\mathrm{C}$ & -5.71459241 & 2.21502323 & 0.91615983 \\
\hline $\mathrm{H}$ & -5.14123089 & 2.51026060 & 1.79050056 \\
\hline $\mathrm{C}$ & -7.07377423 & 1.94507676 & 1.04250835 \\
\hline $\mathrm{H}$ & -7.55564536 & 2.05602559 & 2.00933885 \\
\hline $\mathrm{C}$ & -3.58926329 & 2.22621025 & -0.42896817 \\
\hline $\mathrm{C}$ & -2.90807828 & 3.30775212 & 0.15264292 \\
\hline $\mathrm{H}$ & -3.47603653 & 4.09193974 & 0.64548685 \\
\hline $\mathrm{C}$ & -1.52028600 & 3.40448801 & 0.08603333 \\
\hline $\mathrm{H}$ & -1.03168410 & 4.27181661 & 0.51990781 \\
\hline $\mathrm{C}$ & -0.74748953 & 2.41354200 & -0.54585578 \\
\hline $\mathrm{C}$ & -1.43629570 & 1.34003088 & -1.13810773 \\
\hline $\mathrm{H}$ & -0.87883542 & 0.52795628 & -1.59240346 \\
\hline $\mathrm{C}$ & -2.82213547 & 1.25425231 & -1.09065927 \\
\hline $\mathrm{H}$ & -3.31742563 & 0.38710186 & -1.51559529 \\
\hline $\mathrm{C}$ & 0.73587837 & 2.44637399 & -0.54762342 \\
\hline $\mathrm{C}$ & 1.47842786 & 1.80413387 & -1.55386905 \\
\hline $\mathrm{H}$ & 0.96303791 & 1.34791088 & -2.39275404 \\
\hline $\mathrm{C}$ & 2.86346262 & 1.71828076 & -1.49248328 \\
\hline $\mathrm{H}$ & 3.38476703 & 1.15551549 & -2.25948182 \\
\hline
\end{tabular}




\begin{tabular}{|c|c|c|c|}
\hline $\mathrm{C}$ & 3.59077670 & 2.29330261 & -0.43552786 \\
\hline $\mathrm{C}$ & 2.85325746 & 2.99572451 & 0.53451462 \\
\hline $\mathrm{H}$ & 3.37153711 & 3.49208725 & 1.34898680 \\
\hline $\mathrm{C}$ & 1.46413395 & 3.05978387 & 0.48687695 \\
\hline $\mathrm{H}$ & 0.93520585 & 3.56579022 & 1.28869870 \\
\hline $\mathrm{C}$ & 5.05772045 & 2.09707778 & -0.32006953 \\
\hline $\mathrm{C}$ & 5.85683912 & 1.82598806 & -1.44402814 \\
\hline $\mathrm{H}$ & 5.41842741 & 1.85289219 & -2.43662219 \\
\hline $\mathrm{C}$ & 7.21171195 & 1.53310155 & -1.31574408 \\
\hline $\mathrm{H}$ & 7.80103702 & 1.32494119 & -2.20407680 \\
\hline $\mathrm{C}$ & 7.83151070 & 1.50633786 & -0.06043202 \\
\hline $\mathrm{C}$ & 7.05253734 & 1.83306994 & 1.05688647 \\
\hline $\mathrm{H}$ & 7.51364234 & 1.84595813 & 2.04002333 \\
\hline $\mathrm{C}$ & 5.69577180 & 2.10949946 & 0.93329565 \\
\hline $\mathrm{H}$ & 5.11624704 & 2.29939052 & 1.83128455 \\
\hline $\mathrm{C}$ & -10.10023158 & 4.85308297 & 0.30248415 \\
\hline $\mathrm{H}$ & -10.70418284 & 4.92401648 & 1.21628273 \\
\hline $\mathrm{H}$ & -10.75042616 & 4.99990548 & -0.56939613 \\
\hline $\mathrm{H}$ & -9.34135695 & 5.63684416 & 0.31645128 \\
\hline $\mathrm{H}$ & 9.32214073 & 5.61965976 & 0.33228619 \\
\hline $\mathrm{C}$ & -10.08435705 & -4.83922395 & -0.31622297 \\
\hline $\mathrm{H}$ & -10.68362499 & -4.90699716 & -1.23332226 \\
\hline $\mathrm{H}$ & -10.73810760 & -4.99402198 & 0.55162188 \\
\hline $\mathrm{H}$ & -9.32215327 & -5.61970903 & -0.33129210 \\
\hline $\mathrm{H}$ & 9.34136420 & -5.63677547 & -0.31791735 \\
\hline $\mathrm{O}$ & 9.38672845 & -3.63041469 & -0.23163579 \\
\hline $\mathrm{O}$ & 9.37634467 & 3.61393010 & 0.23465688 \\
\hline $\mathrm{C}$ & 9.30926251 & -1.28950030 & -0.06492342 \\
\hline $\mathrm{C}$ & 10.08017031 & -2.45234094 & -0.18048156 \\
\hline $\mathrm{C}$ & 11.49387220 & -2.42259291 & -0.21436294 \\
\hline $\mathrm{H}$ & 12.06663365 & -3.33698916 & -0.30136915 \\
\hline $\mathrm{C}$ & 12.13385884 & -1.21629089 & -0.10879362 \\
\hline $\mathrm{H}$ & 13.21980009 & -1.17458828 & -0.11155007 \\
\hline $\mathrm{C}$ & 11.41376492 & -0.00503502 & 0.00510763 \\
\hline $\mathrm{C}$ & 12.13108924 & 1.20775017 & 0.12156151 \\
\hline $\mathrm{H}$ & 13.21707864 & 1.16775897 & 0.12925615 \\
\hline $\mathrm{C}$ & 11.48887682 & 2.41318420 & 0.22369013 \\
\hline $\mathrm{H}$ & 12.05929354 & 3.32872043 & 0.31433788 \\
\hline $\mathrm{C}$ & 10.07531881 & 2.43919308 & 0.18257398 \\
\hline $\mathrm{C}$ & 9.30805747 & 1.27483756 & 0.06236093 \\
\hline $\mathrm{C}$ & 9.96729453 & -0.00692405 & 0.00161896 \\
\hline $\mathrm{C}$ & 7.83213206 & -1.51320868 & 0.05468138 \\
\hline
\end{tabular}




\begin{tabular}{|c|c|c|c|}
\hline $\mathrm{C}$ & 7.19029446 & -1.40908780 & 1.29476324 \\
\hline $\mathrm{H}$ & 7.76574423 & -1.11583726 & 2.16792261 \\
\hline $\mathrm{C}$ & 5.82780772 & -1.66483717 & 1.42183140 \\
\hline $\mathrm{H}$ & 5.35111056 & -1.56606563 & 2.39283616 \\
\hline $\mathrm{C}$ & 5.05902449 & -2.05721553 & 0.31566233 \\
\hline $\mathrm{C}$ & 5.71458739 & -2.21513501 & -0.91626262 \\
\hline $\mathrm{H}$ & 5.14122850 & -2.51045429 & -1.79057731 \\
\hline $\mathrm{C}$ & 7.07376703 & -1.94519915 & -1.04264328 \\
\hline $\mathrm{H}$ & 7.55563065 & -2.05624842 & -2.00946547 \\
\hline $\mathrm{C}$ & 3.58926467 & -2.22620264 & 0.42888699 \\
\hline $\mathrm{C}$ & 2.90806595 & -3.30773325 & -0.15273531 \\
\hline $\mathrm{H}$ & 3.47601363 & -4.09190990 & -0.64560846 \\
\hline $\mathrm{C}$ & 1.52027718 & -3.40447886 & -0.08609401 \\
\hline $\mathrm{H}$ & 1.03166649 & -4.27179570 & -0.51998275 \\
\hline $\mathrm{C}$ & 0.74749116 & -2.41355590 & 0.54584467 \\
\hline $\mathrm{C}$ & 1.43630695 & -1.34005859 & 1.13810884 \\
\hline $\mathrm{H}$ & 0.87885221 & -0.52800137 & 1.59244288 \\
\hline $\mathrm{C}$ & 2.82214494 & -1.25426910 & 1.09062465 \\
\hline $\mathrm{H}$ & 3.317433382 & -0.38711978 & 1.51556422 \\
\hline $\mathrm{C}$ & -0.73587577 & -2.44639170 & 0.54764489 \\
\hline $\mathrm{C}$ & -1.47840408 & -1.80421583 & 1.55394695 \\
\hline $\mathrm{H}$ & -0.96299492 & -1.34804463 & 2.39284874 \\
\hline $\mathrm{C}$ & -2.86344137 & -1.71836000 & 1.49259336 \\
\hline $\mathrm{H}$ & -3.38473530 & -1.15564789 & 2.25963883 \\
\hline $\mathrm{C}$ & -3.59077216 & -2.29331371 & 0.43561341 \\
\hline $\mathrm{C}$ & -2.85327333 & -2.99567485 & -0.53448883 \\
\hline $\mathrm{H}$ & -3.37157376 & -3.49198136 & -1.34898256 \\
\hline $\mathrm{C}$ & -1.46414931 & -3.05973883 & -0.48688077 \\
\hline $\mathrm{H}$ & -0.93523225 & -3.56569749 & -1.28874031 \\
\hline $\mathrm{C}$ & -5.05771636 & -2.09707721 & 0.32018732 \\
\hline $\mathrm{C}$ & -5.85683510 & -1.82612938 & 1.44418153 \\
\hline $\mathrm{H}$ & -5.41841958 & -1.85315352 & 2.43677115 \\
\hline $\mathrm{C}$ & -7.21170931 & -1.53323964 & 1.31593016 \\
\hline $\mathrm{H}$ & -7.80104258 & -1.32520058 & 2.20428627 \\
\hline $\mathrm{C}$ & -7.83150737 & -1.50632201 & 0.06061713 \\
\hline $\mathrm{C}$ & -7.05253380 & -1.83290743 & -1.05673914 \\
\hline $\mathrm{H}$ & -7.51363324 & -1.84567135 & -2.03988046 \\
\hline $\mathrm{C}$ & -5.69576429 & -2.10934484 & -0.93317855 \\
\hline $\mathrm{H}$ & -5.11623464 & -2.29911958 & -1.83118918 \\
\hline $\mathrm{C}$ & 10.10023782 & -4.85301630 & -0.30366508 \\
\hline $\mathrm{H}$ & 10.70415829 & -4.92359076 & -1.21751448 \\
\hline $\mathrm{H}$ & 10.75047273 & -5.00020396 & 0.56812650 \\
\hline
\end{tabular}




$\begin{array}{lrrr}\mathrm{C} & 10.08434896 & 4.83918110 & 0.31711566 \\ \mathrm{H} & 10.68358456 & 4.90680649 & 1.23424664 \\ \mathrm{H} & 10.73812760 & 4.99412318 & -0.55068192\end{array}$

Summary of the energies for strain energy calculation (in hartree)

\section{NP-1}

Sum of electronic and zero-point Energies $=$

$-2151.002294$

Sum of electronic and thermal Energies=

$-2150.960297$

Sum of electronic and thermal Enthalpies=

$-2150.959353$

Sum of electronic and thermal Free Energies=

$-2151.076196$

NP-2

Sum of electronic and zero-point Energies $=$

$-3074.936072$

Sum of electronic and thermal Energies=

$-3074.874932$

Sum of electronic and thermal Enthalpies=

$-3074.873988$

Sum of electronic and thermal Free Energies=

$-3075.037176$

SE1

Sum of electronic and zero-point Energies=

Sum of electronic and thermal Energies=

Sum of electronic and thermal Enthalpies=

Sum of electronic and thermal Free Energies=

SE2

Sum of electronic and zero-point Energies=

$-1690.257954$

Sum of electronic and thermal Energies=

$-1690.223215$

Sum of electronic and thermal Enthalpies=

$-1690.222271$

Sum of electronic and thermal Free Energies=

$-1690.328090$

\section{SE3}

Sum of electronic and zero-point Energies=

$-2152.228723$

Sum of electronic and thermal Energies=

$-2152.184693$

Sum of electronic and thermal Enthalpies=

$-2152.183749$

Sum of electronic and thermal Free Energies=

$-2152.311991$ 